\title{
DUALITY FOR COMMUTATIVE GROUP STACKS
}

\author{
SYLVAIN BROCHARD
}

\begin{abstract}
We study in this article the dual of a (strictly) commutative group stack $G$ and give some applications. Using the Picard functor and the Picard stack of $G$, we first give some sufficient conditions for $G$ to be dualizable. Then, for an algebraic stack $X$ with suitable assumptions, we define an Albanese morphism $a_{X}: X \rightarrow A^{1}(X)$ where $A^{1}(X)$ is a torsor under the dual commutative group stack $A^{0}(X)$ of $\mathrm{Pic}_{X / S}$. We prove that $a_{X}$ satisfies a natural universal property. We give two applications of our Albanese morphism. On the one hand, we give a geometric description of the elementary obstruction and of universal torsors (standard tools in the study of rational varieties over number fields). On the other hand we give some examples of algebraic stacks that satisfy Grothendieck's section conjecture.
\end{abstract}

\section{CONTENTS}

1. Introduction

2. Commutative group stacks

3. Dual of a commutative group stack

4. Dualizability

5. Torsors under a commutative group stack

6. A theorem of the square

7. The Albanese torsor

8. Universal properties

9. Application to rational varieties

10. Applications to Grothendieck's section conjecture

11. Some vanishing results for Ext sheaves

References

\section{Introduction}

Motivation. If $X$ is a smooth and projective geometrically connected curve (over a field $k$ ), its Abel-Jacobi morphism $a: X \rightarrow \operatorname{Pic}_{X / k}^{1} \subset \mathrm{Pic}_{X / k}$ is the $X$-point corresponding to the effective divisor $\Delta_{X} \subset X \times_{k} X$. The morphism a maps a point $x$ in $X$ to the class of the line bundle $\mathscr{O}_{X}(x)$. The target $\operatorname{Pic}_{X / k}^{1}$ is a torsor under the jacobian $J=\operatorname{Pic}_{X / k}^{0}$, which is an abelian variety. If $X$ has a $k$-point we get a morphism from $X$ to $J$ itself. This morphism is useful in many contexts: it often allows to reduce some questions (e.g. the injectivity in Grothendieck's section conjecture) to analogous questions about abelian varieties. Now let us consider the following orbifold curve $\mathscr{X}$ over $X$. The morphism $\pi$ is an isomorphism above $X \backslash\{p\}$ and looks

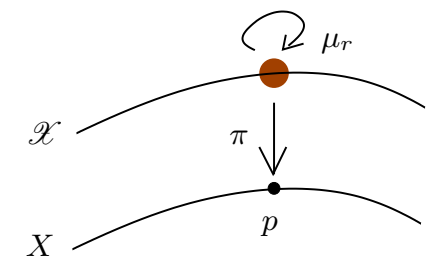

like $\left[\operatorname{Spec}\left(\frac{A[x]}{x^{r}-s}\right) / \mu_{r}\right] \rightarrow \operatorname{Spec} A$ in a neighboorhood $\operatorname{Spec} A$ of $p$. It might be desirable to have 
at our disposal a natural morphism $\mathscr{X} \rightarrow \operatorname{Pic}_{\mathscr{X} / k}$ that could play the role of an Abel-Jacobi morphism. Let us look for such a morphism. Of course we have the obvious composition

$$
\mathscr{X} \stackrel{\pi}{\longrightarrow} X \stackrel{a}{\longrightarrow} \operatorname{Pic}_{X / k} \stackrel{\pi^{*}}{\longrightarrow} \operatorname{Pic}_{\mathscr{X} / k}
$$

but it completely overlooks the stacky structure of $\mathscr{X}$. If we try to mimic the construction of $a$ for $X$ in terms of the diagonal divisor, we come across the fact that $\Delta: \mathscr{X} \rightarrow \mathscr{X} \times_{k} \mathscr{X}$ is not a closed immersion (it is not a monomorphism). Actually, it can be seen (see [17, 5.4]) that there is an exact sequence

$$
0 \longrightarrow \operatorname{Pic}_{X / k} \stackrel{\pi^{*}}{\longrightarrow} \operatorname{Pic}_{\mathscr{X} / k} \longrightarrow \mathbb{Z} / r \mathbb{Z} \longrightarrow 0
$$

so that $\mathrm{Pic}_{\mathscr{X} / k}$ is a disjoint union of $r$ copies of $\mathrm{Pic}_{X / k}$ and $\pi^{*}$ induces an isomorphism $\mathrm{Pic}_{X / k}^{0} \simeq$ $\operatorname{Pic}_{\mathscr{X} / k}^{0}$. But the stack $\mathscr{X}$ itself is connected. So, in some sense, a "natural" morphism from $\mathscr{X}$ to $\operatorname{Pic}_{\mathscr{X} / k}$ must overlook the stacky structure of $\mathscr{X}$.

There is another morphism from $X$ to (a torsor under) an abelian variety: the Albanese morphism. In the case of our curve $X$, it turns out that it coincides with the Abel-Jacobi morphism via the autoduality of the Jacobian $J^{t} \simeq J$. However it is better-behaved than Abel-Jacobi in at least two respects: it is functorial, and it exists for varieties of arbitrary dimension. In the classical setting, the Albanese variety associated to $X$ is dual to the Picard variety $\mathrm{Pic}_{X / k}^{0}$. As the above discussion suggests, to get a meaningful morphism for the stack $\mathscr{X}$, we need to replace the neutral component with the whole Picard functor $\mathrm{Pic}_{\mathscr{X} / k}$ (or at least its torsion component). The latter is not an abelian variety anymore. So this first raises the following question: what is the dual of such a group scheme?

Duality. A dual of an arbitrary abelian sheaf is already defined in the framework of commutative group stacks. Roughly speaking, a commutative group stack (sometimes called a Picard stack) is a stack $G$ together with an addition morphism $\mu: G \times{ }_{k} G \rightarrow G$ that satisfies some natural conditions (see 2.1] and 2.2 below). Deligne has given in SGA 4 3. XVIII 1.4] a description of the 2-category of commutative group stacks in terms of length 1 complexes of sheaves of abelian groups (see 2.5). For example, an abelian sheaf $F$ corresponds to the complex $[0 \rightarrow F]$, the classifying stack $B F$ of $F$ corresponds to $[F \rightarrow 0]$, while a 1-motive (in the sense of Deligne [25]) is a complex $[C \rightarrow A]$ where $C$ is a twisted lattice and $A$ is a semi-abelian scheme. For such a commutative group stack $G$, its dual is the stack of homomorphisms from $G$ to B $\mathbb{G}_{\mathrm{m}}$

$$
D(G)=\mathscr{H} \text { om }\left(G, \mathrm{~B} \mathbb{G}_{\mathrm{m}}\right) .
$$

The duality of Deligne's 1-motives [25, 10.2.10] is a particular case of this construction. For example, if $A$ is an abelian variety, the dual $D(A)$ is the classical dual abelian variety, the dual of $\mathbb{Z}$ is $\mathrm{B} \mathbb{G}_{\mathrm{m}}$, the dual of $\mathbb{G}_{\mathrm{m}}$ is $B \mathbb{Z}$. More generally, if $F$ is a finite flat, a diagonal, or a constant group scheme, then $D(F)=B F^{D}$ and $D(B F)=F^{D}$, where $F^{D}=\operatorname{Hom}\left(F, \mathbb{G}_{\mathrm{m}}\right)$ denotes the Cartier dual of $F$. For any commutative group stack, there is a canonical evaluation map

$$
e_{G}: G \longrightarrow D(D(G)) \text {. }
$$

If $e_{G}$ is an isomorphism, we say that $G$ is dualizable. It seems natural to look for sufficient conditions for a commutative group stack $G$ to be dualizable. Of course Deligne's 1-motives are dualizable. Some other stable classes of dualizable commutative group stacks, inspired from 1motives, have been used recently (see for instance [12, 20] [27, [26], 37, [38] and [48]), e.g. the class of commutative group stacks that are, locally on $S$, finite products of copies of $\mathbb{Z}, \mathrm{B} \mathbb{G}_{\mathrm{m}}$ or abelian schemes (such commutative group stacks are sometimes called Beilinson 1-motives).

Two kinds of geometric conditions can be taken on the commutative group stack $G$, depending on how we think about it. One possibility is to require that there exists a presentation of $G$ as the stack associated to a length one complex $\left[G^{-1} \rightarrow G^{0}\right]$, where the sheaves $G^{-1}$ and $G^{0}$ satisfy certain conditions (this is what happens for 1-motives and the above-mentioned generalizations). Another possibility is to impose directly geometric conditions on the stack $G$, regardless of its presentations. It more or less amounts to impose conditions on the cohomology sheaves $H^{i}\left(G^{\bullet}\right)$ for a length one complex $G^{\bullet}$ that represents $G$ (see for instance 2.14). In this spirit, we conjecture the following: 
Conjecture 1.1. Let $S$ be a base scheme with $2 \in \mathscr{O}_{S}^{\times}$. Let $G$ be a proper, flat, and finitely presented algebraic commutative group stack over $S$, with finite and flat inertia stack. Then:

(1) $D(G)$ is algebraic, proper, flat and finitely presented, with finite diagonal.

(2) $G$ is dualizable.

We get partial results in this direction: under the assumptions we prove that (2) is a consequence of (1) (see 4.14), that $D(G)$ is algebraic and finitely presented with quasi-finite diagonal (3.14 and 3.15), and we prove the conjecture with the additional assumption that $H^{0}(G)$ is cohomologically flat (3.17 and 4.13). We give other results with the same flavour in sections 3 and 4 The assumption that $2 \in \mathscr{O}_{S}^{\times}$is there because of the use of the results of Section [1] and might be superfluous.

Albanese morphism. Now let us come back to our original goal: generalize the Albanese morphism to the context of algebraic stacks. This is done in section 7 Let $f: X \rightarrow S$ be an algebraic stack such that $\mathscr{O}_{S} \rightarrow f_{*} \mathscr{O}_{X}$ is universally an isomorphism (i.e. for any morphism $T \rightarrow S$, the morphism $\mathscr{O}_{T} \rightarrow\left(f_{T}\right)_{*} \mathscr{O}_{X_{T}}$ is an isomorphism, where $f_{T}: X_{T} \rightarrow T$ is the morphism obtained from $f$ after the base change $T \rightarrow S$ ) and such that $f$ locally has sections in the fppf topology on $S$. Then we define a commutative group stack $A^{0}(X)$, a torsor $A^{1}(X)$ under $A^{0}(X)$ and a canonical morphism $a_{X}: X \rightarrow A^{1}(X)$. If we assume that the torsion component of the Picard functor $\operatorname{Pic}_{X / S}^{\tau}$ is proper, flat, cohomologically flat and finitely presented over $S$, then our Albanese morphism is universal among morphisms from $X$ to torsors under what we call "abelian stacks" (see 8.1). Hence it generalizes the classical Albanese morphism given in FGA VI, théorème 3.3 (iii) 33 , exp. 236] in the classical setting of schemes. Note by the way that even in that classical setting, this yields a nice explicit construction of the Albanese torsor and morphism, while the existence proof of FGA was not constructive. The construction of these objects is very simple and natural. Let us denote by $\mathscr{P} i c(X / S)$ the Picard stack of $X$. Then the torsor $A^{1}(X)$ appears as a substack of the dual $D(\mathscr{P} i c(X / S))$, and the morphism $a_{X}$ maps a point $x \in X(S)$ to the pullback morphism $x^{*}: \mathscr{P} i c(X / S) \rightarrow \mathrm{BG}_{\mathrm{m}}$. The universal property 8.1 mentioned above is one of the main results of the paper. In practice, our assumption that $\mathrm{Pic}_{X / S}^{\tau}$ is representable more or less requires $X$ to be at least proper and flat. Note however that, unlike what happens for generalizations of the Albanese mapping to other contexts (e.g. for an arbitrary variety over a field, see for instance [8, 45], 28, 47], 48; note that some of them consider morphisms into non-compact group schemes, which causes difficulties very different from ours), we do not need to assume that $X$ has an $S$-point to get our Albanese morphism, and the Albanese morphism is defined over the whole of $X$ (compare with [8, 7.3]). Also, we do not need to assume that the base scheme $S$ is the spectrum of a field.

For the example of the orbifold curve from the beginning of this introduction, the Albanese stack $A^{0}(\mathscr{X})$ is a gerbe over $A^{0}(X)$ banded by $\mu_{r}$, so that it does remember of the stacky structure of the orbifold curve $\mathscr{X}$. This example is discussed in more details in 8.7 (see also 8.6 which is very similar).

Applications. In [10], Borne and Vistoli have extended Nori's theory of the fundamental group scheme to a theory of the fundamental gerbe, which applies to algebraic stacks even in absence of a rational point. When it exists (for a given algebraic stack $X$ over a field $k$ ), the fundamental gerbe is a morphism $X \rightarrow \Pi_{X / k}$ to a profinite gerbe, with a universal property for morphisms to finite stacks (see [10, 5.1]). Their formalism allows to reformulate Grothendieck's section conjecture as follows: the traditional "section map" is bijective if and only if the natural morphism $X \rightarrow \Pi_{X / k}$ induces a bijection on isomorphism classes of $k$-rational points, in which case we will say that GSC holds for $X$. In this paper we prove that GSC holds for some root stacks over Severi-Brauer varieties. As explained in [10, this result yields a general method for producing examples of smooth projective curves of genus at least 2 that satisfy GSC. We also prove that, if the torsion component $\operatorname{Pic}_{X / k}^{\tau}$ is finite, then the Albanese torsor $A^{1}(X)$ coincides with the abelianization of the fundamental gerbe.

As another application, we give a geometric description of some classical arithmetical constructions. Let $X$ be a proper and smooth variety over a field $k$ and assume that $X$ is $\bar{k}$-rational. Assume that $X$ has "universal torsors" in the sense of Colliot-Thélène and Sansuc (we recall the 
definition in section 9). If the field $k$ is of finite type over its prime subfield, then by [21] there are up to isomorphism finitely many universal torsors $\pi_{i}: T^{i} \rightarrow X(i=1 \ldots n)$ and they provide a partition of the set of $k$-rational points of $X$ :

$$
X(k)=\coprod_{i} \pi_{i}\left(T^{i}(k)\right) .
$$

This description of the $k$-points is useful because, heuristically, the arithmetic of the universal torsors should be much simpler than the arithmetic of the variety $X$. We explain in section 9 how these universal torsors can be described in terms of the Albanese torsor $A^{1}(X)$. In this case $A^{0}(X) \simeq B G_{0}$ where $G_{0}$ is the Cartier dual of $P i c_{X / k}$, which means that $A^{1}(X)$ can be seen as a $G_{0}$-gerbe. We prove moreover that the "elementary obstruction" - an obstruction to the existence of universal torsors - vanishes if and only if the gerbe $A^{1}(X)$ is trivial.

Contents. In section 2, we briefly recall the definition of commutative group stacks and their description in terms of length one complexes. We also give preliminary results about some classes of commutative group stacks that will be used all along the paper. The dual of a commutative group stack is defined in section 3 . Then we start computing the duals of some stacks, compare the dual $D(G)$ with the Picard stack of $G$ and use this comparison to get the main representability theorem mentioned above (3.14). The reader will also find along the way some results that might have independant interest (e.g. Raynaud's devissage 3.11 for proper and flat commutative group schemes over Artin local rings).

In section 4, we address the question of the dualizability, that is: for a given commutative group stack $G$, is $e_{G}: G \rightarrow D D(G)$ an isomorphism? We give a positive answer for some classes of stacks, mostly with properness assumptions (see 4.11, 4.13). This proves that the 2-functor $D($. induces a 2-antiequivalence for these classes of group stacks.

Sections 5 and 6 are devoted to necessary preliminary discussions before dealing with the Albanese morphism. The former recalls basic facts about torsors under a commutative group stack and gerbes banded by a sheaf of commutative groups, for the convenience of the reader, while the latter provides a variant of the famous "theorem of the square" for abelian stacks and for classifying stacks.

Section 7 is devoted to the definition and first properties of the Albanese torsor and Albanese morphism. In section 8 we prove its universal properties and compute some examples.

Sections 9 and 10 are devoted to applications, respectively to rational varieties and in the context of the section conjecture.

The last section 11 recollects for the convenience of the reader some known results about $\mathscr{E} x t$ sheaves that are used throughout the paper.

Notations and terminology. Throughout the paper, the topology we use is the fppf topology, unless we explicitly use another one. Let $S$ be a base scheme. If $X$ is a stack over $S$, we denote by $\mathscr{P} i c(X / S)$ its Picard stack, that is, the stack whose fiber category over an $S$-scheme $U$ is the category of invertibles sheaves on $X \times{ }_{S} U$. The Picard functor is the fppf sheafification of $U \mapsto \operatorname{Pic}\left(X \times{ }_{S} U\right)$ and is denoted by $\operatorname{Pic}_{X / S}$. The coarse moduli sheaf (or coarse moduli space) of $X$ is the fppf sheafification of the presheaf of isomorphism classes of objects of $X$, e.g. Pic $X / S$ is the coarse moduli space of $\mathscr{P} i c(X / S)$. It comes with a universal property, see [39, (3.19)]. A morphism $f: X \rightarrow Y$ is said to be cohomologically flat in dimension zero (or just cohomologically flat, for short) if the formation of $f_{*} \mathscr{O}_{X}$ commutes with base change. If $G$ is a sheaf of abelian groups over $S$, the sheaf $\operatorname{Hom}_{S-\text { gp }}\left(G, \mathbb{G}_{\mathrm{m}}\right)$ of group homomorphisms from $G$ to $\mathbb{G}_{\mathrm{m}}$ is denoted by $G^{D}$ and is called the Cartier dual of $G$. The fppf sheaf $\mathscr{E} x t^{i}\left(G, \mathbb{G}_{\mathrm{m}}\right)$ will often be shortened as $E^{i}(G)$. We denote by $G^{0}$ and $G^{\tau}$ respectively the neutral and torsion component of $G$ (see e.g. [18, 3.3.1]). If $A$ is an abelian scheme - i.e. a smooth and proper group scheme with connected fibers - we denote by $A^{t}$ its dual $\mathrm{Pic}_{A / S}^{0}$. It is an abelian scheme, isomorphic to $E^{1}(A)$ (11.4) and the isomorphism will often be used implicitly. We found it convenient to give (perhaps uncommon) names to some objects. We hope that it will not bother the reader. Here is a list of those terms with the place where the definition can be found: commutative group stack (2.2), dual of a commutative group stack (3.1), abelian stack (2.15), duabelian group (2.17), Cartier group (3.7), evaluation map $e_{G}$ (4.1), $H^{-1}(G)$ and $H^{0}(G)$ (2.9), exact sequence of commutative group stacks (2.11). 
Acknowledgments. I warmly thank Michel Raynaud for Proposition 3.11, and Laurent MoretBailly who first gave me the idea of introducing the map $x \mapsto x^{*}$. I am also grateful to Jean Gillibert, Bertrand Toën, and Olivier Wittenberg for helpful conversations or comments. The content of section 10 started out as joint work with Niels Borne and Angelo Vistoli, whom I thank heartily. I thank the anonymous referees for their very careful reading of the paper and their numerous comments and corrections that greatly clarified the exposition. The author was partially supported by ANR Arivaf (ANR-10-JCJC 0107).

\section{Commutative Group stacks}

In order to fix some notations, and for the convenience of the reader, we recall here very briefly the basics about commutative group stacks, from SGA 4 [3, XVIII 1.4]. These are the stacks called "champs de Picard strictement commutatifs" in [3]. For the details, the reader is refered to the original source. Then we define a notion of exact sequence of commutative group stacks, and two classes of commutative group stacks or sheaves that will be used in the sequel. Note that the group law is denoted additively in this section, but sometimes in the paper it will be more convenient to use a multiplicative notation.

Definition 2.1. A Picard category is a groupoid $G$ (i.e. a category in which all morphisms are isomorphisms) together with a functor

$$
+: G \times G \longrightarrow G,
$$

a functorial associativity isomorphism

$$
\lambda_{x, y, z}:(x+y)+z \stackrel{\sim}{\longrightarrow} x+(y+z),
$$

and a functorial commutativity isomorphism

$$
\tau_{x, y}: x+y \stackrel{\sim}{\longrightarrow} y+x
$$

satisfying the following properties:

(1) the pentagon axiom and the hexagon axiom (see [3, XVIII 1.4.1]),

(2) for any objects $x, y$ of $G, \tau_{y, x} \circ \tau_{x, y}=i d_{x+y}$, and $\tau_{x, x}=i d_{x+x}$,

(3) for any object $x$ of $G$ the functor $y \mapsto x+y$ is an equivalence of categories.

Definition 2.2. Let $S$ be a scheme. A commutative group stack over $S$ is an $S$-stack $G$ together with a morphism $+: G \times{ }_{S} G \rightarrow G$, and 2-isomorphisms $\lambda, \tau$ as above, such that for any $S$ scheme $U$, the fiber category $G(U)$ equipped with the restrictions of,$+ \lambda$ and $\tau$ is a Picard category.

Remark 2.3. A commutative group stack $G$ always has a neutral object, i.e. a pair $(e, \varepsilon)$ where $e$ is an object of $G(S)$ and $\varepsilon$ is an isomorphism $e+e \rightarrow e$. Moreover, this neutral object is unique up to a unique isomorphism, and is neutral on the left and right in a natural sense (see [3], XVIII 1.4.4]). For a fixed neutral object $(e, \varepsilon)$, there is an inverse morphism $-: G \rightarrow G$ which is unique up to a unique 2-isomorphism (see [39, 14.4.1]).

Definition 2.4. Commutative group stacks over $S$ naturally form a 2-category (CGS) as follows. Let $G, H$ be two commutative group stacks over $S$. A homomorphism from $G$ to $H$ is a morphism of stacks $f: G \rightarrow H$ with a 2-isomorphism $\alpha_{f}: f \circ+\Rightarrow+\circ(f \times f)$ such that the following diagrams commute:

$$
\begin{aligned}
& f(x+y) \stackrel{\alpha_{f}}{\longrightarrow} f(x)+f(y) \quad f((x+y)+z) \stackrel{\alpha_{f}}{\longrightarrow} f(x+y)+f(z) \stackrel{\alpha_{f}+i d}{\longrightarrow}(f(x)+f(y))+f(z)
\end{aligned}
$$

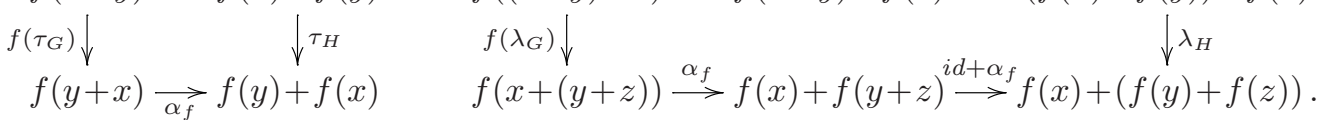

If $f, g$ are homomorphisms from $G$ to $H$, a 2-isomorphism $u: f \Rightarrow g$ is a 2-isomorphism between the underlying morphisms of stacks, that is compatible with $\alpha_{f}$ and $\alpha_{g}$ (see [3, XVIII 1.4.6]). We denote by $\operatorname{Hom}_{c g s}(G, H)$ the category of homomorphisms from $G$ to $H$. 
Let us denote by $\overline{(\mathrm{CGS})}$ the category whose objects are commutative group stacks and whose morphisms are isomorphism classes of homomorphisms. There is a very convenient description of (CGS) and $\overline{(\mathrm{CGS})}$ in terms of length 1 complexes of sheaves of abelian groups, as follows. Let $G^{\bullet}=\left[G^{-1} \rightarrow G^{0}\right]$ be a length 1 complex of sheaves of abelian groups. We denote by $\operatorname{ch}\left(G^{\bullet}\right)$ the quotient stack $\left[G^{0} / G^{-1}\right]$. It is naturally a commutative group stack over $S$. Let $D^{[-1,0]}(S, \mathbb{Z})$ denote the derived category of length 1 complexes of sheaves of abelian groups over $S$ (equivalently, this is the full subcategory of the derived category of sheaves on $S$ of complexes which are homologically concentrated in degrees -1 and 0 ).

Theorem 2.5 (3, XVIII, 1.4.15 and 1.4.17]).

(1) The functor $\mathrm{ch}($.$) induces an equivalence of categories from D^{[-1,0]}(S, \mathbb{Z})$ to $\overline{(C G S)}$. In the sequel we denote by $G \mapsto G^{b}$ a quasi-inverse to this functor.

(2) Let $C(S)$ denote the 2-category of complexes of abelian sheaves $G^{\bullet}$ over $S$, such that $G^{i}=0$ for $i \notin[-1,0]$ and $G^{-1}$ is injective. Morphisms are morphisms of complexes and 2-morphisms are homotopies. Then ch (.) induces a 2-equivalence of 2-categories from $C(S)$ to $(C G S)$.

Example 2.6. Let $G$ be an abelian sheaf over $S$. We can regard it as a commutative group stack (with trivial automorphism groups). Then $G^{\mathrm{b}} \simeq[0 \rightarrow G]$. We can also look at the classifying stack $B G$ of $G$ (which classifies $G$-torsors). Then $(B G)^{b} \simeq[G \rightarrow 0]$.

Example 2.7. Let $X$ be an algebraic stack over $S$. Then the Picard stack $\mathscr{P} i c(X / S)$ is a commutative group stack, and $\mathscr{P}_{i c}(X / S)^{b} \simeq \tau_{\leq 0}\left(R f_{*} \mathbb{G}_{\mathrm{m}}[1]\right)$ where $f: X \rightarrow S$ is the structural morphism of $X$ and where, for a complex $K^{\bullet}$ with differential $\partial^{i}: K^{i} \rightarrow K^{i+1}$, the truncation $\tau_{\leq 0}\left(K^{\bullet}\right)$ is the complex given by $\tau_{\leq 0}\left(K^{\bullet}\right)^{i}=K^{i}$ if $i<0, \tau_{\leq 0}\left(K^{\bullet}\right)^{i}=0$ if $i>0$ and $\tau_{\leq 0}\left(K^{\bullet}\right)^{0}=$ $\operatorname{Ker} \partial^{0}$

Example 2.8. Let $G, H$ be two commutative group stacks over $S$. Then the stack of homomorphisms $U \mapsto \operatorname{Hom}_{c g s}\left(G \times{ }_{S} U, H \times{ }_{S} U\right)$ is naturally a commutative group stack, denoted by $\mathscr{H}$ om $(G, H)$. Then by [3, XVIII 1.4.18], $\mathscr{H}$ om $(G, H)^{b} \simeq \tau_{\leq 0} \operatorname{RHom}\left(G^{b}, H^{b}\right)$.

Definition 2.9. If $G$ is a commutative group stack over $S$, we denote by $H^{0}(G)$ its coarse moduli sheaf (which is an abelian $S$-sheaf) and by $H^{-1}(G)$ the automorphism group of a neutral section of $G$. Note that, for any complex $G^{\bullet}$ as above, there are canonical isomorphisms $H^{i}\left(G^{\bullet}\right) \rightarrow H^{i}\left(\operatorname{ch}\left(G^{\bullet}\right)\right)$.

The following useful proposition is stated without proof in [20].

Proposition 2.10 ([20, A.2.2]). Let $j: A \rightarrow B$ and $\pi: B \rightarrow C$ be two morphisms of commutative group stacks over a base scheme $S$. The following are equivalent:

(1) The morphism $\pi$ is an fppf epimorphism, and $j$ identifies A with the kernel of $\pi$ (i.e. the fiber of $\pi$ above the neutral object of $C$ ).

(2) There exists a morphism $C^{b} \rightarrow A^{b}[1]$ in $D(S, \mathbb{Z})$ (the derived category of abelian sheaves on $S$ ) such that

is an exact triangle.

$$
A^{b} \rightarrow B^{b} \rightarrow C^{b} \rightarrow A^{b}[1]
$$

Proof. Assume (2). The exact triangle induces a long exact sequence

$$
0 \rightarrow H^{-1}(A) \rightarrow H^{-1}(B) \rightarrow H^{-1}(C) \rightarrow H^{0}(A) \rightarrow H^{0}(B) \rightarrow H^{0}(C) \rightarrow 0
$$

In particular $H^{0}(B) \rightarrow H^{0}(C)$ is an fppf epimorphism, hence $\pi: B \rightarrow C$ is an epimorphism. To prove that $A$ is the kernel of $\pi$, we will use the 2-equivalence $2.5(2)$. Let $B^{\bullet}=\left[B^{-1} \stackrel{d_{B}}{\longrightarrow} B^{0}\right]$ and $C^{\bullet}=\left[C^{-1} \stackrel{d_{C}}{\longrightarrow} C^{0}\right]$ be complexes in $C(S)$ corresponding to $B$ and $C$ (in particular $B^{-1}$ and $C^{-1}$ are injective) and let $\pi^{\bullet}$ be a morphism of complexes corresponding to $\pi$. Since we have the given exact triangle, we may assume that $A^{b}[1]$ is the mapping cone of $\pi^{\bullet}$, i.e. in degrees -1 , 0 and $1, A^{b}$ is the complex

$$
B^{-1} \stackrel{d_{A}^{-1}}{\longrightarrow} B^{0} \oplus C^{-1} \stackrel{d_{A}^{0}}{\longrightarrow} C^{0}
$$


where $d_{A}^{-1}=\left(d_{B},-\pi^{-1}\right)$ and $d_{A}^{0}=\left(-\pi^{0}\right) \oplus\left(-d_{C}\right)$, and the other terms are zero. This complex is quasi-isomorphic to $A^{\bullet}=\left[B^{-1} \stackrel{d_{A}^{-1}}{\longrightarrow} \operatorname{Ker} d_{A}^{0}\right]$, which belong to $C(S)$. Now the morphism $j: A \rightarrow B$ corresponds to the morphism of complexes $j^{\bullet}: A^{\bullet} \rightarrow B^{\bullet}$ where $j^{-1}$ is the identity of $B^{-1}$ and $j^{0}$ is the composition $\operatorname{Ker} d_{A}^{0} \hookrightarrow B^{0} \oplus C^{-1} \rightarrow B^{0}$. Let $h: \operatorname{Ker} d_{A}^{0} \rightarrow C^{-1}$ be the projection to $C^{-1}$. Then we have the relations $h d_{A}^{-1}=-\pi^{-1} j^{-1}$ and $d_{C} h=-\pi^{0} j^{0}$ hence $h$ is a homotopy from $\pi^{\bullet} j^{\bullet}$ to 0 . In other words, in $C(S)$ we have a 2-commutative diagram

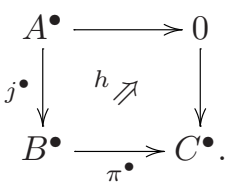

We will prove that this diagram is 2-cartesian. In view of the 2-equivalence 2.5 (2) this will prove that $A$ is the kernel of $\pi$. We have to prove the following assertions:

(i) For any object $D^{\bullet}$ of $C(S)$ and any pair $\left(f^{\bullet}, \alpha\right)$, where $f^{\bullet}: D^{\bullet} \rightarrow B^{\bullet}$ is a morphism in $C(S)$ and $\alpha$ is a homotopy from $\pi^{\bullet} f^{\bullet}$ to 0 , there exists a morphism $g^{\bullet}: D^{\bullet} \rightarrow A^{\bullet}$ and a homotopy $\beta$ from $f^{\bullet}$ to $j^{\bullet} g^{\bullet}$ such that $\alpha=\left(g^{\bullet} \star h\right) \circ\left(\pi^{\bullet} \star \beta\right)$ (viewed as 2-morphisms in the 2-category $C(S)$ ), i.e. such that $\alpha=h g^{0}+\pi^{-1} \beta$ in terms of homotopies (i.e. morphisms from $D^{0}$ to $\left.C^{-1}\right)$.

(ii) For a given $\left(f^{\bullet}, \alpha\right)$, the pair $\left(g^{\bullet}, \beta\right)$ is unique up to a unique isomorphism. More precisely if $\left(g^{\mathbf{}^{\bullet}}, \beta^{\prime}\right)$ is another such pair, then there exists a unique homotopy $\gamma$ from $g^{\bullet}$ to $g^{\prime \bullet}$ such that $\left(j^{\bullet} \star \gamma\right) \circ \beta=\beta^{\prime}$, i.e. such that $j^{-1} \gamma+\beta=\beta^{\prime}$.
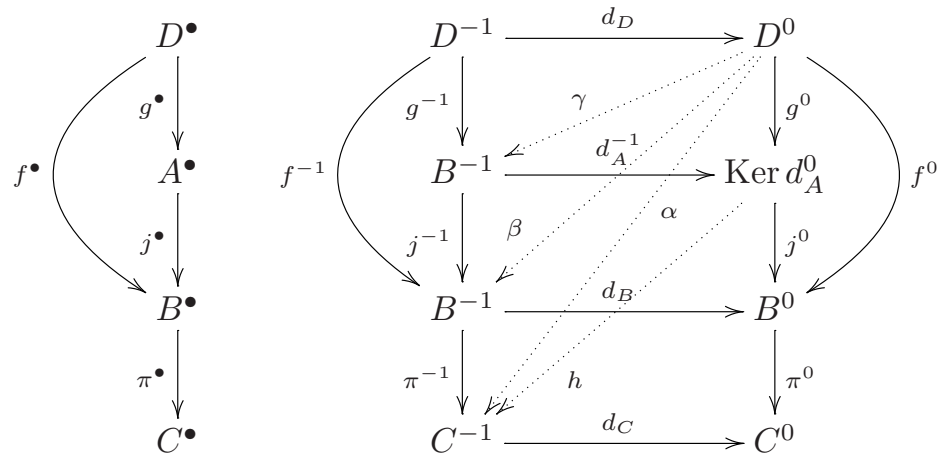

Let us first prove (ii). Since $j^{-1}=$ id we only have to check that $\gamma:=\beta^{\prime}-\beta$ is indeed a homotopy from $g^{\bullet}$ to $g^{\bullet}$, i.e. that it satisfies $\gamma d_{D}=g^{\prime-1}-g^{-1}$ and $d_{A}^{-1} \gamma=g^{\prime 0}-g^{0}$. The first of these relations follows from $\beta d_{D}=j^{-1} g^{-1}-f^{-1}$ and $\beta^{\prime} d_{D}=j^{-1} g^{\prime-1}-f^{-1}$. The second one follows from the equalities

$$
\begin{gathered}
j^{0} d_{A}^{-1} \gamma=d_{B} j^{-1} \gamma=d_{B}\left(\beta^{\prime}-\beta\right)=\left(j^{0} g^{\prime 0}-f^{0}\right)-\left(j^{0} g^{0}-f^{0}\right)=j^{0}\left(g^{\prime 0}-g^{0}\right), \text { and } \\
h d_{A}^{-1} \gamma=-\pi^{-1} j^{-1} \gamma=\pi^{-1}\left(\beta-\beta^{\prime}\right)=\left(\alpha-h g^{0}\right)-\left(\alpha-h g^{\prime 0}\right)=h\left(g^{\prime 0}-g^{0}\right) .
\end{gathered}
$$

Now let us prove (i). Let $\left(f^{\bullet}, \alpha\right)$ be a pair as in (i). The morphism $\left(f^{0}, \alpha\right): D^{0} \rightarrow B^{0} \oplus C^{-1}$ factorizes through $\operatorname{Ker} d_{A}^{0}$ because $d_{C} \alpha=-\pi^{0} f^{0}$ (since $\alpha$ is a homotopy from $\pi^{\bullet} f^{\bullet}$ to 0 ). Then the pair $\left(g^{\bullet}, \beta\right)$ defined by $g^{-1}=f^{-1}, g^{0}=\left(f^{0}, \alpha\right)$ and $\beta=0$ has the required properties. This finishes the proof of (1).

Conversely, assume (1). Let $C_{\pi}$ denote the mapping cone of $\pi^{b}: B^{b} \rightarrow C^{b}$. Then $H^{0}\left(C_{\pi}\right)$ is the cokernel of $H^{0}(B) \rightarrow H^{0}(C)$, which is zero since $\pi: B \rightarrow C$ is an epimorphism. Hence $C_{\pi}$ is concentrated in degrees $[-2,-1]$, so that $C_{\pi}[-1]$ belongs to $D^{[-1,0]}(S, \mathbb{Z})$ and we can consider its associated commutative group stack $\operatorname{ch}\left(C_{\pi}[-1]\right)$. Since we have an exact triangle

$$
C_{\pi}[-1] \rightarrow B \rightarrow C \rightarrow C_{\pi}
$$

by the above we know that $\operatorname{ch}\left(C_{\pi}[-1]\right)$ is the kernel of $\pi: B \rightarrow C$, hence it is isomorphic to $A$. The result follows. 
Definition 2.11. (1) A sequence of commutative group stacks $0 \rightarrow A \rightarrow B \rightarrow C \rightarrow 0$ is said to be exact if the equivalent conditions of 2.10 hold.

(2) The sequence is called super-exact if moreover the connecting homomorphism from $\mathrm{H}^{-1}(C)$ to $H^{0}(A)$ is zero, i.e. if both sequences of group sheaves

$$
0 \longrightarrow H^{i}(A) \longrightarrow H^{i}(B) \longrightarrow H^{i}(C) \longrightarrow 0
$$

are exact $(i=-1,0)$.

Remark 2.12. If $A, B, C$ are sheaves of groups, the definition coincides with the usual notion of an exact sequence. However in the general case, be careful that the morphism $A \rightarrow B$ does not need to be a monomorphism for the sequence to be exact. For instance if $G$ is any (nonzero) sheaf then the kernel of the trivial morphism $S \rightarrow B G$ is $G \rightarrow S$ and the sequence $G \rightarrow S \rightarrow B G$ is exact. Similarly the kernel of the identity $B G \rightarrow B G$ is the trivial morphism $S \rightarrow B G$, which is not a monomorphism either (in this latter example, the sequence $S \rightarrow B G \rightarrow B G$ is even super-exact). Note also that the exactness of both $H^{i}(\bullet)$ sequences does not imply the exactness of the sequence $0 \rightarrow A \rightarrow B \rightarrow C \rightarrow 0$. It does if you assume moreover that $H^{-1}(C)=0$, in which case the sequence is actually super-exact. This will often be the case in that paper.

Example 2.13. For any commutative group stack $G$ over $S$, there is a canonical short exact sequence:

$$
0 \longrightarrow B H^{-1}(G) \stackrel{j}{\longrightarrow} G \stackrel{\pi}{\longrightarrow} H^{0}(G) \longrightarrow 0,
$$

corresponding to the exact triangle $0 \rightarrow H^{-1}(G)[1] \rightarrow G^{b} \rightarrow H^{0}(G) \rightarrow 0$.

Proposition 2.14 (abelian stacks). Let $G$ be a commutative group stack over a base scheme $S$. The following are equivalent:

(1) $G$ is algebraic, proper, flat, and of finite presentation, with connected and reduced (geometric) fibers. Its inertia stack $I_{G}$ is finite, flat and of finite presentation over $G$.

(2) $H^{-1}(G)$ is a finite, flat and finitely presented group scheme. $H^{0}(G)$ is an abelian scheme.

Proof. By definition of $I_{G}$ and $H^{-1}(G)$, there is a commutative diagram with cartesian squares

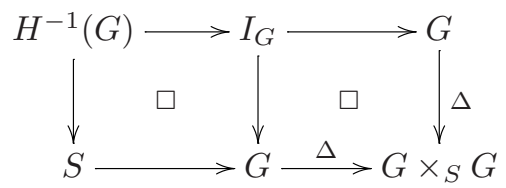

where the morphism $S \rightarrow G$ corresponds to some neutral element $e$ of $G$. This proves that the condition on $I_{G}$ in (1) implies the condition on $H^{-1}(G)$ in (2). Conversely, assume that $H^{-1}(G)$ is finite, flat and finitely presented. Then for any object $g$ of $G$, the "translation by

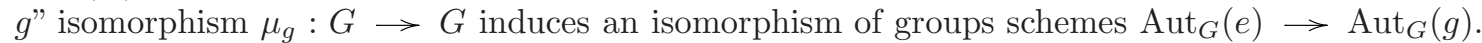
Since $\operatorname{Aut}_{G}(e)=H^{-1}(G)$, this proves that $I_{G}$ is finite, flat and of finite presentation over $G$. Hence the condition on $I_{G}$ in (1) is equivalent to the condition on $H^{-1}(G)$ in $(2)$.

Under this condition, by [39, 10.8], the coarse moduli sheaf $H^{0}(G)$ is an algebraic space and the morphism $\pi: G \rightarrow H^{0}(G)$ is faithfully flat and locally of finite presentation. So $G$ is proper, flat, and of finite presentation if and only if the same holds for $H^{0}(G)$. It remains to prove that the fibers of $H^{0}(G)$ are connected and reduced if and only if those of $G$ are. This is true for the connectedness since $\pi: G \rightarrow H^{0}(G)$ induces a homeomorphism of the underlying topological spaces. If the fibers of $G$ are reduced, by 32 EGA $I_{2} 2.1 .13$ so are those of $H^{0}(G)$. Conversely assume (2) and let us prove that the geometric fibers of $G$ are reduced. By 32 EGA $\operatorname{IV}_{2}$ 6.6.1, it suffices to prove that the geometric fibers of $\pi$ are reduced. This follows from the fact that, if $F$ is a finite commutative group scheme over a field, then the stack $B F$ is reduced. (It is algebraic by [39, 10.13.1]. Then use [32 EGA $\mathrm{IV}_{2}$ 2.1.13.)

Definition 2.15. An abelian stack is a commutative group stack that satisfies the equivalent conditions of 2.14 .

Proposition 2.16 (duabelian groups). Let $G$ be a sheaf of commutative groups over a base scheme $S$. The following are equivalent: 
(1) $G$ is a proper, flat, cohomologically flat and finitely presented algebraic space.

(2) $G$ is an extension of a finite, flat and finitely presented group scheme $F$ by an abelian scheme A. In particular it is a scheme.

Proof. Assume (1) and let us prove (2). Let $f: G \rightarrow S$ be the structural morphism. Then the $\mathscr{O}_{S}$-module $f_{*} \mathscr{O}_{G}$ is of finite type and flat (because $f$ is proper and cohomologically flat). Hence $G_{\mathrm{af}}:=\operatorname{Spec}\left(f_{*} \mathscr{O}_{G}\right)$ is finite and flat. By $\operatorname{SGA} 3$ [1, $\mathrm{VI}_{B}$ 11.3.1] (beware that we use the new edition of SGA 3), there is a group structure on $G_{\mathrm{af}}$ such that the canonical morphism $\rho: G \rightarrow G_{\text {af }}$ is a homomorphism. This latter morphism $\rho$ is finitely presented because $G$ is. It is moreover faithfully flat: this can be checked on the fibers (fiberwise flatness criterion) and over a field it follows from SGA 3 [1, $\mathrm{VI}_{B}$ 12.2]. We use here the fact that, since $f$ is cohomologically flat, the formation of $G_{\mathrm{af}}$ commutes with any base change. Let $N$ denote the kernel of $\rho$. Since $G_{\mathrm{af}}$ is separated the inclusion $N \rightarrow G$ is a closed immersion so $N$ is proper. It is faithfully flat and finitely presented because $\rho$ is, and it has smooth and connected fibers by SGA 3 [1, $\mathrm{VI}_{B} 12.2$ ]. Hence $N$ is an abelian algebraic space. It remains to prove that $G$ is a scheme. By a theorem of Raynaud, we already know that $N$ is a scheme (see [29, I, Theorem 1.9]). Since the question is Zariski-local on $S$, we may assume that $G_{\text {af }}$ is free of rank $n$. It is then killed by $n$, and the $n$-power map $n: G \rightarrow G$ yields an fppf epimorphism $G \rightarrow N$ whose kernel $F$ is finite. In particular $G \rightarrow N$ is finite, hence $G$ is a scheme and this finishes the proof of (2).

Conversely assume (2). Let $\pi: G \rightarrow F$ be the given fppf epimorphism with kernel $A$. Then $G$ is an $A$-torsor over $F$, so by descent it is a proper, flat and finitely presented algebraic space (over $F$, hence also over $S$ ). Since $F$ is cohomologically flat (because it is affine) and $\mathscr{O}_{F} \rightarrow \pi_{*} \mathscr{O}_{G}$ is universally an isomorphism (by descent, and because an abelian scheme has this property) it follows that $G$ is cohomologically flat over $S$, whence (1).

Definition 2.17. A duabelian group is a sheaf of commutative groups that satisfies the equivalent conditions of 2.16 .

We will see later (3.17) that duabelian groups are precisely the duals of abelian stacks.

Proposition 2.18. Let $G$ be a duabelian group scheme over a base scheme $S$. Then $G^{D}$ is finite, flat and finitely presented and $\mathscr{E} x t^{1}\left(G, \mathbb{G}_{\mathrm{m}}\right)$ is an abelian scheme.

Proof. There is an exact sequence $0 \rightarrow A \stackrel{i}{\rightarrow} G \rightarrow F \rightarrow 0$ where $A$ is an abelian scheme and $F$ is a finite and flat group scheme. The statement is local so we may assume that $F$ is free of constant rank $n$. Since $A^{D}=0$ this sequence induces an isomorphism $F^{D} \simeq G^{D}$ hence $G^{D}$ is a finite and flat group scheme. Moreover, since $E^{1}(F)=0$ by 11.1, the morphism $E^{1}(i): E^{1}(G) \rightarrow E^{1}(A)$ is a monomorphism. On the other hand, since $F$ is killed by $n$ by [50, §1], the $n$-power map $[n]_{G}: G \rightarrow G$ induces a morphism $\pi: G \rightarrow A$. The composition $\pi \circ i$ is the fppf epimorphism $[n]_{A}: A \rightarrow A$. Its kernel ${ }_{n} A$ is a finite and flat group scheme over $S$ hence $E^{1}\left({ }_{n} A\right)=0$ and $E^{1}\left([n]_{A}\right): E^{1}(A) \rightarrow E^{1}(A)$ is an epimorphism. Since $E^{1}\left([n]_{A}\right)=E^{1}(i) \circ E^{1}(\pi)$ this proves that $E^{1}(i)$ is an epimorphism. Now $E^{1}(i)$ is both a monomorphism and an epimorphism of fppf sheaves, hence it is an isomorphism and this proves that $E^{1}(G)$ is an abelian scheme.

\section{Dual of a Commutative Group stack}

Definition 3.1. Let $G$ be a commutative group stack over a base scheme $S$. We define its dual to be the stack of homomorphisms of commutative group stacks (2.8) from $G$ to $B \mathbb{G}_{\mathrm{m}}$.

$$
D(G)=\mathscr{H} \text { om }\left(G, B \mathbb{G}_{\mathrm{m}}\right) .
$$

Remark 3.2. If $\varphi: G \rightarrow H$ is a homomorphism of commutative group stacks, the composition with $\varphi$ defines a homomorphism $D(\varphi): D(H) \rightarrow D(G)$. If $\varphi^{\prime}$ is another such homomorphism, any 2-isomorphism $\alpha: \varphi \Rightarrow \varphi^{\prime}$ induces a 2-isomorphism $D(\alpha): D(\varphi) \Rightarrow D\left(\varphi^{\prime}\right)$. This makes $D($. a strict 2-functor from the 2-category of commutative group stacks to itself. Note that $D($.$) is$ additive on maps in the following sense. If $\varphi_{1}, \varphi_{2}$ are homomorphisms from $G$ to $H$, then there is a functorial isomorphism $D\left(\varphi_{1}+\varphi_{2}\right) \simeq D\left(\varphi_{1}\right)+D\left(\varphi_{2}\right)$ of additive homomorphisms from $D(H)$ to $D(G)$ in $\operatorname{Hom}_{c g s}(D(H), D(G))$. 
Remark 3.3. Forming the dual of a commutative group stack commutes with base change: for $G$ as above and for any morphism of schemes $S^{\prime} \rightarrow S$, there is a canonical isomorphism $D\left(G \times{ }_{S}\right.$ $\left.S^{\prime}\right) \simeq D(G) \times{ }_{S} S^{\prime}$.

The next lemma gives a description of the group sheaves $H^{-1}(D(G))$ and $H^{0}(D(G))$ attached to the dual $D(G)$ in terms of those attached to $G$.

Lemma 3.4. Let $G$ be a commutative group stack. Then:

a) $H^{-1}(D(G)) \simeq H^{0}(G)^{D}$;

b) There is an exact sequence:

$$
0 \rightarrow E^{1}\left(H^{0}(G)\right) \rightarrow H^{0}(D(G)) \rightarrow H^{-1}(G)^{D} \rightarrow E^{2}\left(H^{0}(G)\right) .
$$

(Recall that $G^{D}$ denotes the sheaf $\operatorname{Hom}\left(G, \mathbb{G}_{\mathrm{m}}\right)$ of group homomorphisms and $E^{i}(G)$ denotes the fppf sheaf $\mathscr{E} x t^{i}\left(G, \mathbb{G}_{\mathrm{m}}\right)$.)

Proof. By 2.8. $D(G)^{b} \simeq \tau_{\leq 0} \operatorname{RHom}\left(G^{b}, \mathbb{G}_{\mathrm{m}}[1]\right)$. Hence for $i=-1$ or $i=0, H^{i}(D(G)) \simeq$ $\mathscr{E} x t^{i+1}\left(G^{b}, \mathbb{G}_{\mathrm{m}}\right)$. For $i=-1$ this yields $H^{-1}(D(G)) \simeq \operatorname{Hom}\left(H^{0}(G), \mathbb{G}_{\mathrm{m}}\right)$, whence a). For $i=0$ we get $H^{0}(D(G)) \simeq \mathscr{E} x t^{1}\left(G^{b}, \mathbb{G}_{\mathrm{m}}\right)$. There is a spectral sequence (see e.g. [7, Tag 07A9]):

$$
E_{2}^{p, q}=E^{p}\left(H^{-q}\left(G^{b}\right)\right) \Rightarrow \mathscr{E} x t^{p+q}\left(G^{b}, \mathbb{G}_{\mathrm{m}}\right) .
$$

The low degree exact sequence associated to this spectral sequence yields b).

Corollary 3.5. Let $G$ be a sheaf of commutative groups on $S$. Then there is a canonical isomorphism of commutative group stacks

$$
D(B G) \stackrel{\sim}{\longrightarrow} G^{D}
$$

that maps a point $\varphi: B G \rightarrow B \mathbb{G}_{\mathrm{m}}$ of $D(B G)$ to the point $H^{-1}(\varphi): G \rightarrow \mathbb{G}_{\mathrm{m}}$ of $G^{D}$ (using the canonical isomorphisms $H^{-1}(B G) \simeq G$ and $\left.H^{-1}\left(B \mathbb{G}_{\mathrm{m}}\right) \simeq \mathbb{G}_{\mathrm{m}}\right)$.

Proof. We have $H^{0}(B G)=0$ and $H^{-1}(B G)=G$ hence, by the lemma, the exact sequence 2.13 for $D(B G)$ reduces to the desired isomorphism.

Corollary 3.6. Let $G$ be a sheaf of commutative groups on $S$ and regard it as a commutative group stack. Then $H^{-1}(D(G)) \simeq G^{D}$ and $H^{0}(D(G)) \simeq E^{1}(G)$. In particular there is a canonical homomorphism

$$
B G^{D} \longrightarrow D(G) .
$$

This is an isomorphism if and only if the sheaf $E^{1}(G)$ is zero. Note that this morphism can be described as follows: if $T$ is a $G^{D}$-torsor, its image in $D(G)$ is the morphism $G \rightarrow B \mathbb{G}_{\mathrm{m}}$ that maps a point $g$ of $G$ to the $\mathbb{G}_{\mathrm{m}}$-torsor $c(g)_{*} T$ where $c(g): G^{D} \rightarrow \mathbb{G}_{\mathrm{m}}$ is the evaluation at $g$.

Proof. This is a consequence of 3.4. The canonical morphism is the morphism $j$ of 2.13 .

Example 3.7. Recall that if $G$ is a (commutative) constant group scheme, we say that it is finitely generated if the ordinary group that defines the constant group scheme is a finitely generated abelian group. This is not equivalent to $G$ being of finite type. We say that a sheaf of abelian groups $G$ is a finitely generated twisted constant group if, fppf-locally on $S$, it is a finitely generated constant group scheme. Let $G$ be a sheaf of abelian groups that, fppf-locally on $S$, is built up by successive extensions from diagonalizable groups of finite type, finitely generated commutative constant groups, and finite locally free commutative group schemes. Such a group will be called a Cartier group scheme (or we will say that $G$ is Cartier). Recall that groups of multiplicative type and of finite type, and finitely generated twisted constant groups, are actually étale-locally trivial (SGA 3 [2, X, 4.5 and 5.9]). By induction on the number of extensions, we deduce from 11.1 and 11.6 that $E^{1}(G)=0$ and that the Cartier dual $G^{D}$ is still Cartier. (See [2, X, $\S 5$ ] for the duality theory of groups of multiplicative type and of twisted constant groups, and see [49, §4] for Cartier duality of finite locally free group schemes.) In particular, by 3.6 we see that $D(G) \simeq B G^{D}$. On the other hand, if $A$ is an abelian scheme over $S$, then $D(A) \simeq A^{t}$, the classical dual of $A$ as an abelian scheme (use 11.4). 
Example 3.8. If $G$ is a 1-motive, that is, if $G^{\text {b }}$ is quasi-isomorphic to a complex of the form $\left[G^{-1} \rightarrow G^{0}\right]$ where $G^{-1}$ is a twisted lattice and $G^{0}$ is a semi-abelian variety, then $D(G)$ is the classical dual of $G$ as a 1-motive, as described in [25. More generally, if $G^{-1}$ is a sheaf such that $E^{1}\left(G^{-1}\right)=0$, and $G^{0}$ fits into an exact sequence $0 \rightarrow F \rightarrow G^{0} \rightarrow A \rightarrow 0$ where $A$ is an abelian scheme and $E^{1}(F)=0$, then $D(G)^{b}$ is quasi-isomorphic to a complex $\left[F^{D} \rightarrow \widetilde{G^{0}}\right]$ where $\widetilde{G^{0}}$ fits into an exact sequence $0 \rightarrow G^{-1^{D}} \rightarrow \widetilde{G^{0}} \rightarrow A^{t} \rightarrow 0$. We will not need this fact in the sequel, except in the particular case where $G^{-1}=0$. For the convenience of the reader we include a short proof in this case below.

Lemma 3.9. Let $G$ be a sheaf of abelian groups over a base scheme $S$. Assume that there is an exact sequence:

$$
0 \longrightarrow F \longrightarrow G \longrightarrow A \longrightarrow 0
$$

where $A$ is an abelian scheme over $S$ and $E^{1}(F)=0$. Let $\delta: F^{D} \rightarrow A^{t}$ be the natural map given by the $\operatorname{Hom}\left(., \mathbb{G}_{\mathrm{m}}\right)$ sequence. Then the stack $D(G)$ is naturally isomorphic to the quotient stack $\left[A^{t} / F^{D}\right]$ where $F^{D}$ acts on $A^{t}$ via $\delta$.

Proof. By 2.5 it suffices to prove that $D(G)^{b} \simeq\left[F^{D} \rightarrow A^{t}\right]$ in the derived category $D^{[-1,0]}(S, \mathbb{Z})$. By [3, XVIII 1.4.18], $D(G)^{b} \simeq \tau_{\leq 0} \operatorname{RHom}\left(G, \mathbb{G}_{\mathrm{m}}[1]\right)$. Viewing the given exact sequence as a triangle in $D^{[-1,0]}(S, \mathbb{Z})$ and applying the functor $\operatorname{RHom}\left(., \mathbb{G}_{\mathrm{m}}[1]\right)$, we get a triangle:

$$
\operatorname{RHom}\left(A, \mathbb{G}_{\mathrm{m}}[1]\right) \rightarrow \operatorname{RHom}\left(G, \mathbb{G}_{\mathrm{m}}[1]\right) \rightarrow \operatorname{RHom}\left(F, \mathbb{G}_{\mathrm{m}}[1]\right) \rightarrow \operatorname{RHom}\left(A, \mathbb{G}_{\mathrm{m}}[2]\right) .
$$

Let $C=\operatorname{RHom}\left(F, \mathbb{G}_{\mathrm{m}}[1]\right)$. Since, by [3.6, $H^{0}(C)=E^{1}(F)=0$, we can truncate the above triangle in degrees $\leq 0$ using the Lemma 3.10 below. Since moreover the truncations of $\operatorname{RHom}\left(A, \mathbb{G}_{\mathrm{m}}[1]\right)$ and $\operatorname{RHom}\left(F, \mathbb{G}_{\mathrm{m}}[1]\right)$ are $A^{t}$ and $F^{D}[1]$ we get a triangle:

$$
F^{D} \stackrel{\delta}{\longrightarrow} A^{t} \longrightarrow \tau_{\leq 0} \operatorname{RHom}\left(G, \mathbb{G}_{\mathrm{m}}[1]\right) \longrightarrow F^{D}[1]
$$

This proves that $\tau_{\leq 0} \operatorname{RHom}\left(G, \mathbb{G}_{\mathrm{m}}[1]\right)$ is isomorphic to the cone of $\delta$, which is precisely the complex $\left[F^{D} \rightarrow A^{t}\right]$.

Lemma 3.10. Let $\mathscr{A}$ be an abelian category and let $A \stackrel{u}{\longrightarrow} B \stackrel{v}{\longrightarrow} C \longrightarrow A[1]$ be a triangle in $D(\mathscr{A})$. Then $H^{n}(B) \rightarrow H^{n}(C)$ is surjective (i.e. its cokernel is zero) if and only if there exists a morphism $w: \tau_{\leq n} C \rightarrow\left(\tau_{\leq n} A\right)[1]$ such that

$$
\tau_{\leq n} A \longrightarrow \tau_{\leq n} B \longrightarrow \tau_{\leq n} C \longrightarrow\left(\tau_{\leq n} A\right)[1]
$$

is an exact triangle.

Proof. If we have an exact triangle as above, the associated long exact sequence proves that $H^{n}(B) \rightarrow H^{n}(C)$ is surjective. Conversely, assume that $H^{n}(B) \rightarrow H^{n}(C)$ is surjective and let us construct the desired $w$. For this we may assume that $C$ is the mapping cone of $u$, i.e. $C^{i}=B^{i} \oplus A^{i+1}$ for all $i$. Let $C_{\tau_{\leq n} u}$ denote the mapping cone of $\tau_{\leq n} u$. There is a natural morphism $\alpha: C_{\tau_{\leq n} u} \rightarrow \tau_{\leq n} C$ given in degree $n-1$ (resp. $n$ ) by the inclusion $B^{n-1} \oplus \operatorname{Ker}\left(A^{n} \rightarrow A^{n+1}\right) \hookrightarrow$ $B^{n-1} \oplus A^{n}$ (resp. $\left.\operatorname{Ker}\left(B^{n} \rightarrow B^{n+1}\right) \hookrightarrow \operatorname{Ker}\left(C^{n} \rightarrow C^{n+1}\right)\right)$. Then $H^{i}(\alpha)$ is an isomorphism for all $i \neq n$, and $H^{n}(\alpha)$ identifies with the morphism Coker $\left(H^{n}(A) \rightarrow H^{n}(B)\right) \hookrightarrow H^{n}(C)$. Since $H^{n}(B) \rightarrow H^{n}(C)$ is surjective, $H^{n}(\alpha)$ is an isomorphism hence $\alpha$ is a quasi-isomorphism and this concludes the proof.

Proposition 3.11 (Raynaud). Let $S$ be the spectrum of an Artin local ring with algebraically closed residue field $k$, and let $G$ be a proper and flat commutative group scheme over $S$. Then there is an exact sequence

$$
0 \longrightarrow F \longrightarrow G \longrightarrow 0
$$

where $A$ is an abelian scheme and $F$ is a finite flat group scheme.

Proof. We first assume that the field $k$ has characteristic $p>0$. Let us denote by $S_{0}$ the spectrum of $k$ and by $G_{0}$ the reduction of $G$ to $S_{0}$. Since $k$ is algebraically closed, by SGA 3 [1, VI $\mathrm{VI}_{A} 5.5 .1$ and 5.6.1], $G_{0}$ is the extension of a finite $k$-group $M_{0}$ by an abelian scheme $A_{0}$. Let $n$ be an integer that kills $M_{0}$. Then the $n$-power map $[n]: G_{0} \rightarrow G_{0}$ factorizes through $A_{0}$ and yields 
a morphism $u_{0}: G_{0} \rightarrow A_{0}$. Moreover the composition $A_{0} \rightarrow G_{0} \rightarrow A_{0}$ is the isogeny $[n]$ of $A_{0}$, hence it is finite, flat and surjective. This proves that $u_{0}$ is an fppf epimorphism. Let $F_{0}$ be its kernel. Let $K$ be the kernel of $[n]: A_{0} \rightarrow A_{0}$. Then $K$ is also the kernel of the composition $F_{0} \rightarrow G_{0} \rightarrow M_{0}$, which is an fppf epimorphism. Hence $F_{0}$ is an extension of $M_{0}$ by $K$ and is thus finite. So $G_{0}$ is extension of the abelian scheme $A_{0}$ by a finite group scheme.

$$
0 \longrightarrow F_{0} \longrightarrow G_{0} \longrightarrow A_{0} \longrightarrow 0
$$

By a theorem of Grothendieck (see [33] for the statement without proof, a detailed proof is given in [30, 8.5.23]), there exists an abelian scheme $A$ over $S$ lifting $A_{0}$. Now, the morphism $u_{0}$ does not necessarily lift to a morphism of schemes $G \rightarrow A$, but it does if we increase the integer $n$ used above. Indeed, let $S_{0} \rightarrow S_{1} \rightarrow \ldots \rightarrow S_{m}=S$ be a factorization of the morphism $S_{0} \rightarrow S$, where each morphism $S_{i} \rightarrow S_{i+1}$ is an extension of spectra of Artin local rings defined by a square-zero ideal $I_{i+1}$. We denote by a subscript $i$ the objects above $S_{i}$ obtained after the base change $S_{i} \rightarrow S$ from those above $S$. Assume that there exists a morphism of group schemes $u_{i}$ : $G_{i} \rightarrow A_{i}$ lifting $u_{0}$. Then by Illusie [36, VII 3.3.1.1], there is a class $\omega \in H^{1}\left(G_{i}, u_{i}^{*} \Omega_{A_{i} / S_{i}}^{1} \otimes I_{i+1}\right)$ that vanishes if and only if $u_{i}$ lifts to a morphism of schemes $u_{i+1}$. The obstruction to lift $u_{i} \circ\left[p^{\ell}\right]$ is $p^{\ell} . \omega$ hence vanishes for $\ell$ large enough (because the group $H^{1}$ above is actually a $\Gamma\left(S_{i}, \mathscr{O}_{S_{i}}\right)$ module). The resulting morphism of schemes $u_{i+1}: G_{i+1} \rightarrow A_{i+1}$ is not necessarily a group morphism, but once again, for $n$ large enough it is so. (We can also use SGA 3 [1, III 2.1] instead of Illusie.) By induction we get a homomorphism $u: G \rightarrow A$ lifting $u_{0}$. By SGA 1 [5, IV 5.9] $u$ is automatically flat. It is surjective and finite because so is $u_{0}$. This yields the desired exact sequence, with $F$ the kernel of $u$.

If the field $k$ has characteristic zero, this is much easier: let $G^{0}$ denote the connected component of 0 in $G$. Since $G$ is of finite type, $G^{0}$ is an open and closed subscheme of $G$ hence it is proper and flat over $S$. Since the residue field has characteristic zero, the special fiber of $G$ is smooth hence $G$ itself is smooth because it is flat with smooth fibers. This proves that $G^{0}$ is an abelian scheme over $S$. By SGA 3 [1, $\mathrm{VI}_{A}$ 5.5.1], the quotient $G / G^{0}$ is finite and étale, so that $G$ is the extension of a finite étale group by an abelian scheme. Using the $n$-power map $[n]: G \rightarrow G$ as in the beginning of the proof, we directly get the desired exact sequence over $S$.

Corollary 3.12. Let $S$ be the spectrum of an Artin ring $R$ and let $G$ be a proper and flat commutative group scheme over $S$. Then the dual $D(G)$ is proper and flat.

Proof. Since an Artin ring is a product of Artin local rings, we may assume that $R$ is local. Let us prove that there exists a faithfully flat morphism $S^{\prime} \rightarrow S$ where $S^{\prime}$ is the spectrum of an Artin local ring with algebraically closed residue field. We can write $R$ as a quotient of $W\left[X_{1}, \ldots, X_{n}\right]$ for some integer $n$ and some discrete valuation ring $W$ (use e.g. Cohen's theory). By [34, I, 5.5.3] there exists a discrete valuation ring $W^{\prime}$ that dominates $W$ with algebraically closed residue field. Then $R \otimes_{W} W^{\prime}$ is an Artin local ring with algebraically closed residue field and is faithfully flat over $R$. Now by faithfully flat descent, we may assume that the residue field of $R$ is algebraically closed. By 3.11, 11.1 and 3.9, $D(G)$ is isomorphic to a quotient stack $[A / F]$ where $A$ is an abelian scheme acted on by a finite and flat commutative group scheme $F$. By [39, 10.13.1] the stack $[A / F]$ is algebraic. Moreover the canonical morphism $A \rightarrow[A / F]$ is finite and faithfully flat. It follows that $D(G)$ is proper and flat.

Let $G$ be a commutative group stack over a base scheme $S$. If we identify the category of morphisms of stacks from $G$ to $B \mathbb{G}_{\mathrm{m}}$ with the category of invertible sheaves on $G$, we see that there is a natural forgetful morphism

$$
\omega: D(G) \longrightarrow \mathscr{P} i c(G / S) .
$$

We can translate Definition 2.4 in terms of invertible sheaves to give an alternative and useful description of the stack $D(G)$. It will be helpful to study the representability of the stack $D(G)$, see 3.14. Let us first fix some notation. Let Sym : $G \times_{S} G \rightarrow G \times{ }_{S} G$ be the isomorphism that exchanges the two factors, and let $\mu_{G}: G \times{ }_{S} G \rightarrow G$ be the group law in $G$. Let $p_{i}$ (resp. $q_{i}$ ) be the projection of $G \times{ }_{S} G$ (resp. $G \times{ }_{S} G \times{ }_{S} G$ ) onto the $i$-th factor. By definition of $G$, there is a 
2-isomorphism $\tau: \mu_{G} \Rightarrow \mu_{G} \circ$ Sym (commutativity) and a 2-isomorphism of associativity

$$
\lambda: \mu_{G} \circ\left(\mu_{G} \times \operatorname{id}_{G}\right) \Rightarrow \mu_{G} \circ\left(\operatorname{id}_{G} \times \mu_{G}\right) .
$$

If $\mathscr{L}$ is an invertible sheaf on $G$, then $\tau$ and $\lambda$ induce isomorphisms of invertible sheaves on $G \times{ }_{S} G$ (resp. on $G \times{ }_{S} G \times{ }_{S} G$ )

$$
\begin{aligned}
& \tau(\mathscr{L}): \quad \mu_{G}^{*} \mathscr{L} \longrightarrow \operatorname{Sym}^{*} \mu_{G}^{*} \mathscr{L} \\
& \lambda(\mathscr{L}):\left(\mu_{G} \times \operatorname{id}_{G}\right)^{*} \mu_{G}^{*} \mathscr{L} \longrightarrow\left(\operatorname{id}_{G} \times \mu_{G}\right)^{*} \mu_{G}^{*} \mathscr{L} .
\end{aligned}
$$

Now the description of $D(G)$ is as follows. For any $S$-scheme $U$, an object of the fiber category $D(G)(U)$ is a couple $(\mathscr{L}, \alpha)$ where $\mathscr{L}$ is an invertible sheaf on $G \times{ }_{S} U$ and $\alpha$ is an isomorphism

$$
\alpha: \mu_{G}^{*} \mathscr{L} \longrightarrow p_{1}^{*} \mathscr{L} \otimes p_{2}^{*} \mathscr{L}
$$

such that the two following diagrams commute

$$
\begin{aligned}
& \begin{aligned}
& \mu_{G}^{*} \mathscr{L} \longrightarrow p_{1}^{*} \mathscr{L} \otimes p_{2}^{*} \mathscr{L} \\
& \tau(\mathscr{L}) \downarrow \quad{ }_{\downarrow}(A) \quad \downarrow \text { can. }
\end{aligned} \\
& \operatorname{Sym}^{*} \mu_{G}^{*} \mathscr{L} \underset{\operatorname{Sym}^{*} \alpha}{\longrightarrow} \operatorname{Sym}^{*}\left(p_{1}^{*} \mathscr{L} \otimes p_{2}^{*} \mathscr{L}\right) \\
& \left(\mu_{G} \times \operatorname{id}_{G}\right)^{*} \mu_{G}^{*} \mathscr{L} \longrightarrow\left(\operatorname{id}_{G} \times \mu_{G}\right)^{*} \mu_{G}^{*} \mathscr{L} \\
& \left(\mu_{G} \times \operatorname{id}_{G}\right)^{*} \alpha \downarrow \\
& \left(\mu_{G} \times \operatorname{id}_{G}\right)^{*}\left(p_{1}^{*} \mathscr{L} \otimes p_{2}^{*} \mathscr{L}\right) \\
& \text { can. } \downarrow^{2} \\
& \left(\left(q_{1} \times q_{2}\right)^{*} \mu_{G}^{*} \mathscr{L}\right) \otimes q_{3}^{*} \mathscr{L} \\
& \left(\left(q_{1} \times q_{2}\right)^{*} \alpha\right) \otimes \mathrm{id}_{q_{3}^{*} \mathscr{L} \downarrow} \\
& \left(q_{1} \times q_{2}\right)^{*}\left(p_{1}^{*} \mathscr{L} \otimes p_{2}^{*} \mathscr{L}\right) \otimes q_{3}^{*} \mathscr{L} \\
& \downarrow\left(\operatorname{id}_{G} \times \mu_{G}\right)^{*} \alpha \\
& \left(\operatorname{id}_{G} \times \mu_{G}\right)^{*}\left(p_{1}^{*} \mathscr{L} \otimes p_{2}^{*} \mathscr{L}\right) \\
& \text { i } \downarrow \text { can. } \\
& q_{1}^{*} \mathscr{L} \otimes\left(q_{2} \times q_{3}\right)^{*} \mu_{G}^{*} \mathscr{L} \\
& \downarrow \operatorname{id}_{q_{1}^{*} \mathscr{L}} \otimes\left(q_{2} \times q_{3}\right)^{*} \alpha \\
& { }_{q_{1}^{*} \mathscr{L}}^{*} \otimes q_{2}^{*} \mathscr{L} \otimes q_{3}^{*} \mathscr{L}^{\text {can. }}
\end{aligned}
$$

If $x=(\mathscr{L}, \alpha)$ and $x^{\prime}=\left(\mathscr{L}^{\prime}, \alpha^{\prime}\right)$ are two such objects, a morphism $x \rightarrow x^{\prime}$ is an isomorphism $\beta: \mathscr{L} \rightarrow \mathscr{L}^{\prime}$ such that the diagram

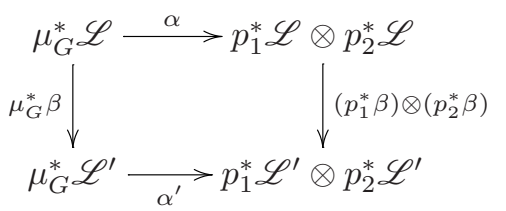

commutes.

Remark 3.13. This description also shows that $D(G)$ is the stack of extensions of $G$ by $\mathbb{G}_{\mathrm{m}}$. To see this, use e.g. [41, I, 2.3.10]. In the language of [41, an isomorphism $\alpha: \mu_{G}^{*} \mathscr{L} \rightarrow p_{1}^{*} \mathscr{L} \otimes p_{2}^{*} \mathscr{L}$ corresponds to a section $\sigma$ of the $\mathbb{G}_{\mathrm{m}}$-torsor $\theta_{2}(\mathscr{L})$ above $G \times{ }_{S} G$. Then the commutativity of the diagram (A) (resp. (B)) is equivalent to the condition I, 2.3 .8 (resp. I, 2.3.9) of [41.

Theorem 3.14. Let $S$ be a base scheme. Let $G$ be an algebraic commutative group stack which is proper, flat and finitely presented over $S$. Then:

(1) The morphism $\omega$ is affine and of finite presentation.

(2) The stack $D(G)$ is algebraic and of finite presentation, with affine diagonal. Its fibers are proper.

(3) If $G$ is an algebraic space (i.e. $H^{-1}(G)=0$ ), then $D(G)$ is flat.

Proof. Let us prove (1). Since $G$ is of finite presentation, by standard limit arguments we can assume that $S$ is noetherian. Let $U$ be an $S$-scheme and $U \rightarrow \mathscr{P} i c(G / S)$ a morphism, corresponding to an invertible sheaf $\mathscr{L}$ on $G \times_{S} U$. By [18, 2.1.1] and 35, Thm D] the sheaf $I=\mathscr{I} \operatorname{som}\left(\mu_{G}^{*} \mathscr{L}, p_{1}^{*} \mathscr{L} \otimes p_{2}^{*} \mathscr{L}\right)$ is an affine scheme of finite presentation over $U$. By the above description, we see that the fiber product $D(G) \times \mathscr{P}_{i c(G / S)} U$ identifies with the closed subspace 
of $I$ defined by the conditions (A) and (B), hence it is also an affine scheme of finite presentation over $U$.

Let us prove (2). By [17, [18, 2.1.1] and [35, Thm D], the stack $\mathscr{P} i c(G / S)$ is algebraic and locally of finite presentation, with affine diagonal. Hence so is $D(G)$ by (1). Let us now prove that $D(G)$ has proper fibers. For this we may assume that $S$ is the spectrum of an algebraically closed field $k$. If $G$ is a group scheme the assertion follows from 3.12. Now, let $G$ be a proper commutative group stack over $k$. Then $H^{-1}(G)$ and $H^{0}(G)$ are duabelian $k$-group schemes (note that a proper group scheme over the spectrum of a field is obviously flat and finitely presented, but also cohomologically flat because the formation of direct images commutes with flat base change). In particular, by 2.18 $H^{-1}\left(D(G)\right.$ ) (which is isomorphic to $H^{0}(G)^{D}$ by 3.4) and $H^{-1}(G)^{D}$ are finite and $E^{1}\left(H^{0}(G)\right)$ is an abelian variety. By Artin [39, 10.8] the coarse moduli sheaf $H^{0}(D(G))$ is an algebraic space locally of finite presentation (hence actually a group scheme) and the projection $\pi: D(G) \rightarrow H^{0}(D(G))$ is faithfully flat and locally of finite presentation. By 3.4 there is an exact sequence

$$
0 \longrightarrow E^{1}\left(H^{0}(G)\right) \stackrel{\delta}{\longrightarrow} H^{0}(D(G)) \longrightarrow H^{-1}(G)^{D} \longrightarrow E^{2}\left(H^{0}(G)\right) .
$$

By SGA 3 [1, $\mathrm{VI}_{A} 3.2$ ] the cokernel $K$ of $\delta$ is a group scheme. But it has a monomorphism to the finite group scheme $H^{-1}(G)^{D}$. Thus $K$ is finite and this proves that $H^{0}(D(G))$ is proper. This implies that $D(G)$ itself is proper, because it is a gerbe over $H^{0}(D(G))$, banded by $H^{-1}(D(G))$ which is finite.

To prove (2) it only remains to prove that $D(G)$ is quasi-compact for a general base $S$. To this end, we use the notion of quasicompactness introduced in [18 for non necessarily representable stacks (or morphisms of stacks). The fibers of $D(G)$ are proper by the above. In particular the morphism $D(G) \rightarrow \operatorname{Pic}_{G / S}$ factorizes through $\mathrm{Pic}_{G / S}^{\tau}$. By [18, 3.3.3], we know that $\mathrm{Pic}_{G / S}^{\tau}$ is quasi-compact over $S$. Moreover the morphism $\mathscr{P}_{i c^{\tau}}(G / S) \rightarrow \operatorname{Pic}_{G / S}^{\tau}$ is an fppf gerbe hence quasi-compact. To conclude, it suffices to prove that the morphism from $D(G)$ to $\mathscr{P} i c^{\tau}(G / S)$ is quasi-compact. By [18, 3.3.3] the inclusion $\mathscr{P}_{i c^{\tau}}(G / S) \rightarrow \mathscr{P}_{i c}(G / S)$ is an open immersion hence its diagonal is quasi-compact. Since $\omega$ is quasi-compact, by [18, 3.1.3 (vii)] we see that $D(G)$ is quasi-compact over $S$ and this finishes the proof of (2).

Since the flatness of a locally noetherian stack can be checked on Artin rings (SGA 1 [5] IV 5.6]), the assertion (3) follows from 3.12

Theorem 3.15. Let $G$ be an algebraic commutative group stack which is proper, flat and finitely presented over a base scheme $S$. Assume that $H^{-1}(G)$ is flat. Then $H^{-1}(D(G))$ is a finite group scheme.

Proof. Since $H^{-1}(G)$ is flat, $H^{0}(G)$ is a proper, flat and finitely presented algebraic space. Since $H^{-1}(D(G))=H^{0}(G)^{D}$ we may assume that $G$ is an algebraic space and we have to prove that $G^{D}$ is finite. By 3.14, we already know that $G^{D}$ is affine and finitely presented, with finite fibers. We now prove that it is proper. By [39, A.2.1] this will imply that it is finite. For this question we can assume that $S$ is the spectrum of a discrete valuation $\operatorname{ring} R$ with fraction field $K$. Let $G_{\text {af }}$ be the spectrum of $f_{*} \mathscr{O}_{G}$. By [4, VII 3.2] and SGA 3 [1, VI $\mathrm{VI}_{B}$ 11.3.1] it is a finite flat group scheme, the canonical morphism $\rho: G \rightarrow G_{\text {af }}$ is a homomorphism, and it is universal for homomorphisms to affine $R$-groups. In particular it induces a bijection $G_{\mathrm{af}}^{D}(R) \rightarrow G^{D}(R)$. Since forming $G_{\mathrm{af}}$ commutes to any flat base change, the natural map $G_{\mathrm{af}}^{D}(K) \rightarrow G^{D}(K)$ is also bijective. Lastly, $G_{\mathrm{af}}^{D}$ is finite hence satisfies the valuative criterion thus $G^{D}$ is finite.

Corollary 3.16. Let $G$ be a proper, flat and finitely presented commutative group algebraic space over a base scheme $S$. Then $G^{D}$ is a finite group scheme.

Proof. This is the particular case of 3.15 where $H^{-1}(G)=0$.

Theorem 3.17. Let $S$ be a base scheme and let $G$ be a commutative group stack over $S$.

(1) If $G$ is a duabelian group, then $D(G)$ is an abelian stack.

(2) If $G$ is an abelian stack, then $D(G)$ is a duabelian group.

(3) Assume that 2 is invertible in $S$. If $H^{0}(G)$ is duabelian and $H^{-1}(G)$ is finite and flat, then $H^{0}(D(G))$ is duabelian and $H^{-1}(D(G))$ is finite and flat. 
Proof. (1) is an immediate consequence of 2.18 and 3.4. Let us prove (2) and (3). There is an exact sequence:

$$
0 \longrightarrow E^{1}\left(H^{0}(G)\right) \longrightarrow H^{0}(D(G)) \longrightarrow H^{-1}(G)^{D} \longrightarrow E^{2}\left(H^{0}(G)\right) .
$$

The group $H^{-1}(G)^{D}$ is finite and flat. By 2.18, $E^{1}\left(H^{0}(G)\right)$ is an abelian scheme. Moreover by 11.5 in both cases the morphism from $H^{-1}(G)^{D}$ to $E^{2}\left(H^{0}(G)\right)$ is zero. Hence $H^{0}(D(G))$ is duabelian and this proves (3). If $G$ is an abelian stack then $H^{0}(G)$ is an abelian scheme. By 11.4 its Cartier dual vanishes hence $D(G)=H^{0}(D(G))$ and it is a duabelian group.

It is a natural question to ask whether the above duality operation preserves short exact sequences. In general this is not the case. The following proposition gives a positive answer with suitable assumptions.

Proposition 3.18. Let $(*): 0 \longrightarrow A \stackrel{j}{\longrightarrow} B \stackrel{\pi}{\longrightarrow} C \longrightarrow 0$ be a short exact sequence of commutative group stacks.

(a) The following are equivalent:

(i) The dual sequence $D(*)$ is exact.

(ii) The morphism of stacks $D(j): D(B) \rightarrow D(A)$ is an epimorphism.

(iii) The morphism of sheaves $H^{0}(D(B)) \rightarrow H^{0}(D(A))$ is an epimorphism.

(b) If $E^{2}\left(H^{0}(C)\right)=E^{1}\left(H^{-1}(C)\right)=0$, then $D(*)$ is exact.

(c) Assume that $(*)$ is super-exact. If $E^{2}\left(H^{0}(C)\right)=0$ and if the morphism from $H^{-1}(A)^{D}$ to $E^{1}\left(H^{-1}(C)\right)$ vanishes, then $D(*)$ is exact.

(d) Assume that $(*)$ is super-exact and that $D(*)$ is exact. Then $D(*)$ is super-exact if and only if the morphism $H^{0}(A)^{D} \rightarrow E^{1}\left(H^{0}(C)\right)$ is zero.

Proof. (a) The implications $(i) \Rightarrow($ ii $) \Rightarrow($ iii $)$ are obvious, and $(i i i) \Rightarrow(i)$ follows from 3.10 .

(b) The exact triangle

$$
\operatorname{RHom}\left(C^{b}, \mathbb{G}_{\mathrm{m}}[1]\right) \rightarrow \operatorname{RHom}\left(B^{b}, \mathbb{G}_{\mathrm{m}}[1]\right) \rightarrow \operatorname{RHom}\left(A^{b}, \mathbb{G}_{\mathrm{m}}[1]\right) \rightarrow \operatorname{RHom}\left(C^{b}, \mathbb{G}_{\mathrm{m}}[2]\right)
$$

induces an exact sequence $H^{0}(D(B)) \rightarrow H^{0}(D(A)) \rightarrow \operatorname{Ext}^{2}\left(C^{b}, \mathbb{G}_{\mathrm{m}}\right)$. By [7, Tag 07A9]) there is a spectral sequence:

$$
E_{2}^{p, q}=E^{p}\left(H^{-q}\left(C^{b}\right)\right) \Rightarrow \operatorname{Ext}^{p+q}\left(C^{b}, \mathbb{G}_{\mathrm{m}}\right) .
$$

Since $C^{b}$ is concentrated in degrees $-1,0$, we have $E_{2}^{p, q}=0$ for $q \geq 2$ and the spectral sequence yields a long exact sequence :

$$
\ldots \rightarrow E^{2}\left(H^{0}(C)\right) \rightarrow \operatorname{Ext}^{2}\left(C^{b}, \mathbb{G}_{\mathrm{m}}\right) \rightarrow E^{1}\left(H^{-1}(C)\right) \rightarrow \ldots
$$

so that $\operatorname{Ext}^{2}\left(C^{b}, \mathbb{G}_{\mathrm{m}}\right)=0$.

The assertions (c) and (d) are proven by chasing through the commutative diagram

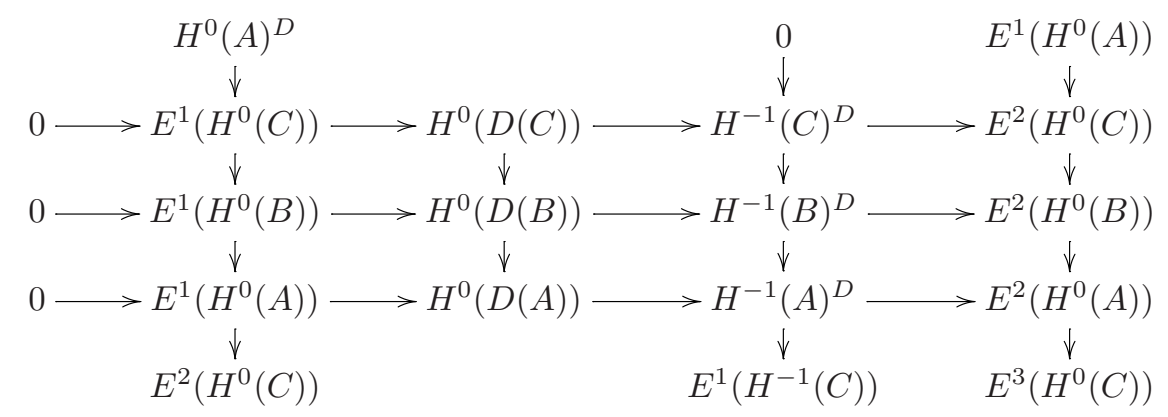

in which the rows (obtained from Lemma 3.4 ), and the first, third and fourth columns are exact.

Remark 3.19. Let $G$ be an arbitrary commutative group stack. Apply 3.18 to the canonical short exact sequence 2.13 i.e. with $A, B$ and $C$ respectively equal to $B H^{-1}(G), G$ and $H^{0}(G)$. Then $H^{0}(A)=0$ and $H^{-1}(C)=0$. By 3.18 a) the dual sequence $D(2.13$ is exact if and only 
if $H^{0}(D(B)) \rightarrow H^{0}(D(A))$ is an epimorphism. Using the commutative diagram in the proof of 3.18 we can identify this latter morphism with $H^{0}(D(B)) \rightarrow H^{-1}(B)^{D}$, hence the sequence

$$
0 \longrightarrow D\left(H^{0}(G)\right) \longrightarrow D(G) \longrightarrow D\left(B H^{-1}(G)\right) \longrightarrow 0
$$

is (super-)exact if and only if the natural morphism $H^{-1}(G)^{D} \rightarrow E^{2}\left(H^{0}(G)\right)$ is trivial.

We now give three lemmas that will be used to compare the dual $D(G)$ of an abelian stack with the torsion component $\mathrm{Pic}_{G / S}^{\tau}$ of the Picard functor (see 3.23).

Lemma 3.20. Let $u: G \rightarrow H$ be a morphism of commutative group algebraic spaces over a base scheme $S$. Assume that:

(i) For any geometric point $s:$ Speck $\rightarrow S$ of $S$, the induced morphism $u_{s}$ is an isomorphism.

(ii) $G$ is flat and of finite presentation over $S$.

(iii) $H$ is locally of finite type over $S$.

Then $u$ is an isomorphism.

Proof. Note that SGA $3[1] \mathrm{VI}_{B} 2.10$ and 2.11 also hold for algebraic spaces. Then by $\mathrm{VI}_{B} 2.11 u$ is a monomorphism. Let $Q$ denote the fppf quotient sheaf. By Artin [39, 10.4] it is an algebraic space locally of finite type. Then by [1, $\left.\mathrm{VI}_{B} 2.10\right]$, the group $Q$ is trivial.

Lemma 3.21. Let $S$ be a scheme and let $X$ be an algebraic stack over $S$ such that $X(S)$ is nonempty and $\mathscr{O}_{S} \rightarrow f_{*} \mathscr{O}_{X}$ is an isomorphism (where $f$ is the structural morphism of $X$ ). Let $Y \rightarrow S$ be an affine morphism of schemes. Then any morphism $g: X \rightarrow Y$ is constant, i.e. it factorizes through $f$.

Proof. Let $x \in X(S)$. It suffices to prove that the maps $g$ and $g \circ x \circ f$ are equal. We can assume that $S$ and $Y$ are affine. Now the set $\operatorname{Hom}(X, Y)$ can be identified with $\operatorname{Hom}\left(\Gamma\left(Y, \mathscr{O}_{Y}\right), \Gamma\left(X, \mathscr{O}_{X}\right)\right)$ and the result follows.

Lemma 3.22. Let $G$ be an algebraic commutative group stack. Assume that $\mathscr{O}_{S} \rightarrow f_{*} \mathscr{O}_{G}$ is universally an isomorphism. Then $H^{-1}(D(G))=0$, and the natural morphism from $D(G)$ to $\mathrm{Pic}_{G / S}$ is a monomorphism.

Proof. Let $\varphi: H^{0}(G) \rightarrow \mathbb{G}_{\mathrm{m}}$ be a morphism of group sheaves. By 3.21 the induced morphism from $G$ to $\mathbb{G}_{\mathrm{m}}$ is trivial and this implies that $\varphi$ is trivial. Since the assumptions are stable under base change, it follows that $H^{0}(G)^{D}=0$, hence $H^{-1}(D(G))=0$.

To prove that $D(G) \rightarrow \mathrm{Pic}_{G / S}$ is a monomorphism, it suffices to prove that the induced map from $D(G)(S)$ to $\operatorname{Pic}_{G / S}(S)$ is injective (again because the assumptions are stable under base change). Let $\sigma: G \rightarrow B \mathbb{G}_{\mathrm{m}}$ be an $S$-point of $D(G)$, such that the corresponding invertible sheaf is mapped to 0 in $\operatorname{Pic}_{G / S}(S)$. By [17, 2.2.6] it is mapped to 0 in $\operatorname{Pic}(G) / \operatorname{Pic}(S)$, which means that the morphism of stacks underlying $\sigma$ factorizes through $S$. This in turn implies that the morphism of commutative group stacks $\sigma$ is trivial.

Proposition 3.23. Let $G$ be an abelian stack over a base scheme $S$. Then the forgetful morphism from $D(G)$ to $\mathscr{P} i c(G / S)$ induces a functorial isomorphism

$$
D(G) \stackrel{\sim}{\longrightarrow} \operatorname{Pic}_{G / S}^{\tau} .
$$

Proof. By standard limit arguments we may assume that $S$ is noetherian. The structural morphism $f: G \rightarrow S$ is proper, flat, finitely presented, and with geometrically connected and geometrically reduced fibers (2.14). In particular, $\mathscr{O}_{S} \rightarrow f_{*} \mathscr{O}_{G}$ is universally an isomorphism. Then the Picard functor $\mathrm{Pic}_{G / S}$ is representable by a quasiseparated algebraic space [18, Theorem 2.1.1 (2)] and the subfunctor $\operatorname{Pic}_{G / S}^{\tau}$ is representable by an open subscheme, which is of finite presentation over $S$ [18, Theorem 3.3.3]. On the other hand, by 3.17 $D(G)$ is a proper and flat algebraic space over $S$. The natural morphism $\omega: D(G) \rightarrow \mathrm{Pic}_{G / S}$ is a monomorphism by 3.22 Since $D(G)$ is proper it factorizes through $\operatorname{Pic}_{G / S}^{\tau}$. We still denote by $\omega$ the resulting monomorphism $D(G) \rightarrow \mathrm{Pic}_{G / S}^{\tau}$. To prove that it is an isomorphism, by 3.20 we may assume that $S$ is the spectrum of an algebraically closed field. 
Let us first consider the case where $G$ is an abelian variety. Then, by 3.6, we know that $D(G)$ is isomorphic to the abelian variety $\operatorname{Pic}_{G / S}^{\tau}$ hence $\omega$ is necessarily an isomorphism since it is injective.

Now let us consider the case where $H^{0}(G)=0$. Then $\operatorname{Pic}_{G / S} \simeq H^{-1}(G)^{D}$ (see for instance [17, 5.3.7] or 3.24 below) and the whole Picard functor is torsion. On the other hand, the commutative group stack $D(G)$ is also isomorphic to $H^{-1}(G)^{D}$ by 3.5. The morphism $\omega$ is a proper monomorphism, hence a closed immersion, and since both sides are finite and isomorphic it must be an isomorphism.

In the general case, the canonical exact sequence 2.13 induces a commutative diagram

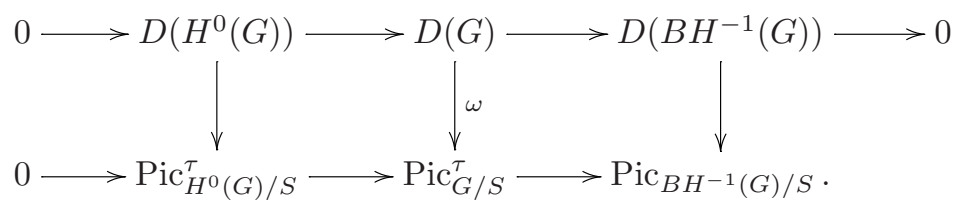

The morphism $H^{-1}(G)^{D} \rightarrow E^{2}\left(H^{0}(G)\right)$ is trivial (11.5), hence the first row is exact by 3.19. The second row is exact too 3.24 and [18, 3.3.2]). The left and right vertical maps are isomorphisms by the above particular cases, hence by the 5-lemma (which holds in any abelian category and in particular in the category of sheaves, see e.g. [40, VIII, $\$ 4$, Lemma4]; note by the way that in loc. cit. the assumptions on $f_{1}$ and $f_{5}$ are too strong: it suffices to have $f_{1}$ epic and $f_{5}$ monic) the middle one is also an isomorphism.

\section{Proposition 3.24.}

(1) Let $F$ be a sheaf of commutative groups over a base scheme $S$. There is a canonical isomorphism $\mathrm{Pic}_{B F / S} \stackrel{\sim}{\longrightarrow} F^{D}$.

(2) Let $F$ be a separated, flat and finitely presented commutative group algebraic space over $S$, and let $\mathscr{G}$ be an $F$-gerbe (see 5.9) over an $S$-scheme $X$. There is an exact sequence:

$$
0 \longrightarrow \operatorname{Pic}_{X / S} \longrightarrow \operatorname{Pic}_{\mathscr{G} / S} \longrightarrow F^{D} \text {. }
$$

Proof. (1) is proved in [17, 5.3.7] (the sheaf $F$ was supposed to be a scheme in loc. cit. but this assumption was useless). The isomorphism maps an invertible sheaf $L$ on $B F$ to the unique character $\chi_{L}: F \rightarrow \mathbb{G}_{\mathrm{m}}$ such that the natural action of $F$ on $L$ is induced through $\chi_{L}$ by that of $\mathbb{G}_{\mathrm{m}}$. The inverse of this isomorphism maps a character $\chi$ to the class of the invertible sheaf $\mathscr{L}(\chi)$ corresponding to the induced map $B F \rightarrow B \mathbb{G}_{\mathrm{m}}$.

(2) By [17, 5.3.6] the sequence $\operatorname{Pic}(X) \rightarrow \operatorname{Pic}(\mathscr{G}) \rightarrow F^{D}(S)$ is exact. Let $\pi: \mathscr{G} \rightarrow X$ denote the structural morphism of $\mathscr{G}$. The Leray spectral sequence of $\pi$ yields an injection $H^{1}\left(X, \pi_{*} \mathbb{G}_{\mathrm{m}}\right) \hookrightarrow H^{1}\left(\mathscr{G}, \mathbb{G}_{\mathrm{m}}\right)$. But the canonical map $\mathscr{O}_{X} \rightarrow \pi_{*} \mathscr{O}_{\mathscr{G}}$ is universally an isomorphism, hence $\pi_{*} \mathbb{G}_{\mathrm{m}}=\mathbb{G}_{\mathrm{m}}$ and we see that $\pi^{*}: \operatorname{Pic}(X) \rightarrow \operatorname{Pic}(\mathscr{G})$ is injective. Sheafifying, we get the exactness of the sequence $0 \rightarrow \operatorname{Pic}_{X / S} \rightarrow \operatorname{Pic}_{\mathscr{G} / S} \rightarrow F^{D}$.

Remark 3.25. We let the reader check that the isomorphisms 3.5 and 3.24 (1) are compatible with the forgetful morphism $\omega: D(B G) \rightarrow \mathrm{Pic}_{B G / S}$ in the sense that the following diagram commutes.

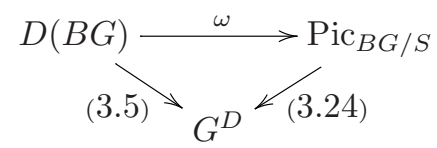

Corollary 3.26. Let $G$ be a sheaf of commutative groups over a base scheme $S$. Then the forgetful morphism from $D(B G)$ to $\mathscr{P} i c(B G / S)$ induces an isomorphism

$$
\omega: D(B G) \stackrel{\sim}{\longrightarrow} \operatorname{Pic}_{B G / S} .
$$

\section{Dualizability}

Definition 4.1. Let $G$ be a commutative group stack over a base scheme $S$. There is a natural evaluation homomorphism

$$
e_{G}: G \longrightarrow D D(G)
$$


that maps a point $g$ of $G$ to the homomorphism $e_{G}(g): D(G) \rightarrow B \mathbb{G}_{\mathrm{m}}$ defined by $e_{G}(g)(\varphi)=\varphi(g)$ for any object $\varphi: G \rightarrow B \mathbb{G}_{\mathrm{m}}$ of $D(G)$. We say that $G$ is dualizable if $e_{G}$ is an isomorphism.

\section{Proposition 4.2.}

(a) The evaluation map $e_{G}$ is functorial in the following sense. If $f: G \rightarrow H$ is a morphism of commutative group stacks, then the square

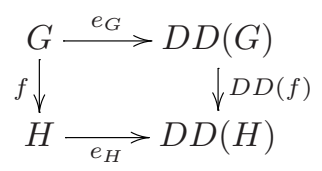

is strictly commutative.

(b) The composition $D\left(e_{G}\right) \circ e_{D(G)}$

$$
D(G) \stackrel{e_{D(G)}}{\longrightarrow} D D D(G) \stackrel{D\left(e_{G}\right)}{\longrightarrow} D(G)
$$

is equal to the identity of $D(G)$.

(c) Let $G$ be a dualizable commutative group stack. Then $D(G)$ is dualizable.

(d) Forming the evaluation morphism commutes with base change.

(e) For commutative group stacks, the property of being dualizable is stable under base change.

Proof. (b) and (d) are straightforward verifications and (c), (e) are immediate consequences. To prove (a), we just observe that both morphisms $e_{H} \circ f$ and $D D(f) \circ e_{G}$ map an object $x$ of $G$ to the morphism of commutative group stacks

$$
\begin{aligned}
\mathscr{H} \text { om }\left(H, B \mathbb{G}_{\mathrm{m}}\right) & \longrightarrow B \mathbb{G}_{\mathrm{m}} \\
\varphi & \longmapsto \varphi(f(x))
\end{aligned}
$$

Proposition 4.3. The 2-functor $D($.$) induces a 2-antiequivalence from the 2-category of dualizable$ commutative group stacks to itself.

Proof. If $G$ is dualizable, then so is $D(G)$, and $G$ is by definition isomorphic to $D(D(G))$, hence $D($.$) is 2-essentially surjective. It remains to prove that for G$ and $H$ dualizable, the functor

$$
D(.): \operatorname{Hom}(G, H) \longrightarrow \operatorname{Hom}(D(H), D(G))
$$

is an equivalence of categories. Using $4.2(\mathrm{a})$, we observe that the functors $D D(.) \circ e_{G}$ and $\left(e_{H} \circ\right.$.) from $\operatorname{Hom}(G, H)$ to $\operatorname{Hom}(G, D D(H))$ are equal. Since $\left(. \circ e_{G}\right)$ and $\left(e_{H} \circ.\right)$ are equivalences, we deduce that $D D($.$) is an equivalence. The result then follows from the lemma below, whose proof$ is straightforward.

Lemma 4.4. Let $F: A \rightarrow B$ and $G: B \rightarrow C$ be functors. Assume that $G \circ F$ is an equivalence. Then:

(i) $F$ is faithful and $G$ is essentially surjective.

(ii) If $F$ is essentially surjective (resp. if $G$ is faithful) then $G$ (resp. $F$ ) is full.

(iii) $F$ is an equivalence if and only if $G$ is an equivalence.

Proposition 4.5. Let $G$ be a commutative group stack over a base scheme $S$. Let $S^{\prime} \rightarrow S$ be an fppf cover. The following are equivalent:

(i) $G$ is dualizable.

(i') The morphisms $H^{i}\left(e_{G}\right): H^{i}(G) \rightarrow H^{i}(D D(G))$ are isomorphisms $(i=-1,0)$.

(ii) $G \times{ }_{S} S^{\prime}$ is dualizable.

(ii') The morphisms $H^{i}\left(e_{G \times S_{S} S^{\prime}}\right)$ are isomorphisms $(i=-1,0)$.

Proof. The equivalences $(i) \Leftrightarrow\left(i^{\prime}\right)$ and $(i i) \Leftrightarrow\left(i i^{\prime}\right)$ follow from Deligne's equivalence between the category of commutative group stacks and the derived category $D^{[-1,0]}(S, \mathbb{Z})$ of length 1 complexes of $f p p f$ sheaves of commutative groups. The equivalence $\left(i^{\prime}\right) \Leftrightarrow\left(i i^{\prime}\right)$ follows from the fact that, for a morphism of fppf sheaves, being an isomorphism is local in the fppf topology. 
Lemma 4.6. Let $G$ be a sheaf of abelian groups. Then the diagrams

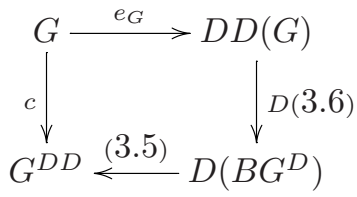

and

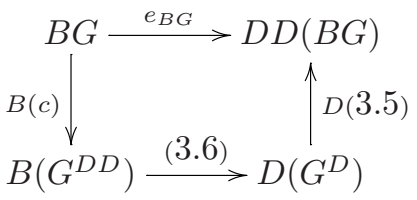

where $c$ stands for the evaluation map of Cartier duality, commute.

Proof. Let us prove that the first diagram commutes. It suffices to do it on $S$-points, so let $g \in G(S)$. On the one hand, $c(g): G^{D} \rightarrow \mathbb{G}_{\mathrm{m}}$ is the morphism that maps an element $\varphi \in G^{D}(S)$ to $\varphi(g) \in \mathbb{G}_{\mathrm{m}}(S)$. The other side is a little bit more complicated to describe: it is the morphism that we get by applying $H^{-1}$ to the morphism $e_{G}(g) \circ$ (3.6). The morphism $H^{-1}$ (3.6) identifies with the canonical isomorphism $G^{D} \simeq H^{-1}(D(G))$, in other words it maps an element $\varphi \in G^{D}(S)$ to the automorphism of the neutral element $e: G \rightarrow B \mathbb{G}_{\mathrm{m}}$ of $D(G)$ that maps a point $h$ of $G$ to the automorphism of the trivial $\mathbb{G}_{\mathrm{m}}$-torsor that corresponds to $\varphi(h)$. It remains to describe $e_{G}(g): D(G) \rightarrow B \mathbb{G}_{\mathrm{m}}$ on automorphisms. Let $\psi: G \rightarrow B \mathbb{G}_{\mathrm{m}}$ be an object of $D(G)$ (actually we are only interested in the neutral object). Then $e_{G}(g)$ maps an automorphism $u$ of $\psi$ (that is, the data, for each element $h$ of $G$ of an automorphism $u(h)$ of $\left.\psi(h) \in B \mathbb{G}_{\mathrm{m}}\right)$ to the automorphism $u(g)$ of $\psi(g)$. In the end, the morphism $G^{D} \rightarrow \mathbb{G}_{\mathrm{m}}$ that we get is indeed equal to the morphism that maps an element $\varphi \in G^{D}$ to $\varphi(g)$. The commutativity of the second diagram is a similar definition-chasing.

Proposition 4.7. Let $G$ be a sheaf of commutative groups over $S$.

(1) The stack $B G$ is dualizable if and only if the natural map $G \rightarrow G^{D D}$ is an isomorphism (in other words, $G$ is dualizable in the sense of Cartier duality) and $E^{1}\left(G^{D}\right)=0$.

(2) If $E^{i}\left(E^{1}(G)\right)=0$ for $i=0,1,2$ and $G \rightarrow G^{D D}$ is an isomorphism, or if $G$ is an abelian scheme, then $G$ is dualizable as a commutative group stack.

Proof. (1) is a consequence of 3.5, 3.6, and 4.6 .

Let us prove (2). Assume first that $E^{i}\left(E^{1}(G)\right)=0$ for $i=0,1,2$ and $G \rightarrow G^{D D}$ is an isomorphism. By 3.18 the dual of the sequence $0 \rightarrow B G^{D} \rightarrow D(G) \rightarrow E^{1}(G) \rightarrow 0$ is exact. Since $E^{i}\left(E^{1}(G)\right)=0$ for $i=0,1$ we see that $D\left(E^{1}(G)\right)=0$. Hence the canonical morphism $D(3.6): D D(G) \rightarrow D\left(B G^{D}\right)$ is an isomorphism and by 4.6 this proves that $e_{G}$ is an isomorphism.

Now let $A$ be an abelian scheme. By 3.23 we know that $D(A)$ and $D D(A)$ are abelian schemes. We let the reader check that the following diagram commutes, which proves that $e_{A}$ is an isomorphism.

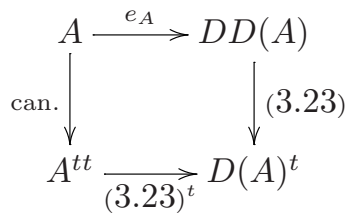

Example 4.8. If $G$ is a Cartier group (see 3.7), then $E^{1}(G)=E^{1}\left(G^{D}\right)=0$ and $G \rightarrow G^{D D}$ is an isomorphism. Hence $G$ and $B G$ are dualizable.

Let $G$ be a commutative group stack over a base scheme $S$. Let us denote $A:=B H^{-1}(G)$ and $C:=H^{0}(G)$. By 2.13 there is a canonical exact sequence $0 \rightarrow A \rightarrow G \rightarrow C \rightarrow 0$. Assume that the morphism $H^{-1}(G)^{D} \rightarrow E^{2}\left(H^{0}(G)\right)$ is trivial. Then by 3.19] the sequence $0 \rightarrow D(C) \rightarrow D(G) \rightarrow D(A) \rightarrow 0$ is super-exact. Applying $\operatorname{Hom}\left(., \mathbb{G}_{\mathrm{m}}\right)$ to the $H^{0}$ sequence we get a morphism $H^{0}(D(C))^{D} \rightarrow E^{1}(D(A))$. But by 3.5. $D(A) \simeq H^{-1}(G)^{D}$ and by 3.4. $H^{0}(D(C)) \simeq E^{1}\left(H^{0}(G)\right)$, so that we get a morphism $E^{1}\left(H^{0}(G)\right)^{D} \rightarrow E^{1}\left(H^{-1}(G)^{D}\right)$.

Proposition 4.9. Let $G$ be a commutative group stack over a base scheme $S$. Assume that the morphism $H^{-1}(G)^{D} \rightarrow E^{2}\left(H^{0}(G)\right)$ is trivial and that the induced morphism (described above) $E^{1}\left(H^{0}(G)\right)^{D} \rightarrow E^{1}\left(H^{-1}(G)^{D}\right)$ is trivial as well. If both $B H^{-1}(G)$ and $H^{0}(G)$ are dualizable, then so is $G$. 
Proof. With the above notations, using the diagram in the proof of 3.18 we see that the sequences $(i=-1,0)$

$$
0 \longrightarrow H^{i}(D D(A)) \longrightarrow H^{i}(D D(G)) \longrightarrow H^{i}(D D(C))
$$

are exact. Hence, for $i=-1,0$ we have a commutative diagram with exact lines:

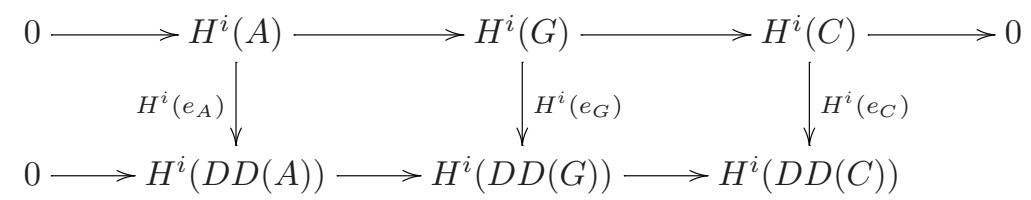

Since the left and right vertical arrows are isomorphisms, so is the middle one.

Example 4.10. Let $G$ be an abelian stack over $S$. By 4.7 $B H^{-1}(G)$ and $H^{0}(G)$ are dualizable. The morphism $H^{-1}(G)^{D} \rightarrow E^{2}\left(H^{0}(G)\right)$ is trivial by 11.5, and $E^{1}\left(H^{0}(G)\right)^{D}=0$ by 11.4 Hence $G$ is dualizable.

Theorem 4.11. Let $S$ be a regular base scheme in which 2 is invertible. Let $G$ be a commutative group stack over $S$. Assume that étale-locally on $S$ :

(i) $H^{0}(G)$ fits in an exact sequence

$$
0 \longrightarrow A \longrightarrow H^{0}(G) \longrightarrow F \longrightarrow 0
$$

where $A$ is an abelian scheme over $S$, and $F$ is built up, by successive extensions, from finite locally free group schemes and constant free group schemes of finite rank, and

(ii) $H^{-1}(G)$ is built up by successive extensions from finite locally free group schemes and split tori.

Then $G$ is dualizable, and $D(G)$ satisfies the same assumptions as $G$. More precisely, as soon as (i) and (ii) hold for $G$, then $H^{-1}(D(G)) \simeq F^{D}$, and $H^{0}(D(G))$ fits in an exact sequence

$$
0 \longrightarrow A^{t} \longrightarrow H^{0}(D(G)) \longrightarrow H^{-1}(G)^{D} \longrightarrow 0 \text {. }
$$

Proof. We can assume that (i) and (ii) hold. By 4.8 since $H^{-1}(G)$ is Cartier, we know that $E^{1}\left(H^{-1}(G)^{D}\right)=0$ and that $B H^{-1}(G)$ is dualizable. By 4.9, to prove that $G$ is dualizable it suffices to prove that $E^{2}\left(H^{0}(G)\right)=0$ and $H^{0}(G)$ is dualizable. The exact sequence given in (i) induces a long exact sequence:

$$
0 \rightarrow F^{D} \rightarrow H^{0}(G)^{D} \rightarrow A^{D} \rightarrow E^{1}(F) \rightarrow E^{1}\left(H^{0}(G)\right) \rightarrow E^{1}(A) \rightarrow E^{2}(F) \ldots
$$

Since the sheaves $A^{D}, E^{1}(F), E^{2}(F)$ and $E^{2}(A)$ all vanish (section 11), we see that $E^{2}\left(H^{0}(G)\right.$ ) is zero and we get isomorphisms $F^{D} \simeq H^{0}(G)^{D}$ and $E^{1}\left(H^{0}(G)\right) \simeq A^{t}$. Using the description of $D\left(H^{0}(G)\right)$ from 3.6 and applying 3.18 twice, we see that the sequence

$$
0 \longrightarrow D D(A) \longrightarrow D D\left(H^{0}(G)\right) \longrightarrow D D(F) \longrightarrow 0
$$

is super-exact. Hence the dualizability of $H^{0}(G)$ follows from that of $A$ and $F$. This proves that $G$ is dualizable. Moreover, by 3.4 we have an isomorphism $H^{-1}(D(G)) \simeq H^{0}(G)^{D}$ and an exact sequence

$$
0 \rightarrow E^{1}\left(H^{0}(G)\right) \rightarrow H^{0}(D(G)) \rightarrow H^{-1}(G)^{D} \rightarrow E^{2}\left(H^{0}(G)\right)=0,
$$

whence the assertions about $D(G)$.

Example 4.12. Assume that the base scheme is the spectrum of an algebraically closed field $k$ of characteristic different from 2 . Let $G$ be an algebraic commutative $k$-group stack locally of finite type. Assume that $\left(H^{-1}(G)\right)_{\text {red }}^{0}$ is a torus and that $\left(H^{0}(G)\right)_{\text {red }}^{0}$ is an abelian variety. Assume moreover that the groups of connected components of $H^{0}(G)$ and $H^{-1}(G)$ are of finite type as ordinary abelian groups. Then by SGA 3 [1, $\mathrm{VI}_{A}$ 5.5.1 and 5.6.1], $G$ satisfies the assumptions of 4.11 hence it is dualizable.

The assumptions on $S$ in 4.11 might be superfluous. We can drop them if we restrict the class of commutative group stacks, using the results of Section 3

Theorem 4.13. Let $G$ be a commutative group stack over a base scheme $S$. Assume that one of the following holds: 
(i) $G$ is a duabelian group.

(ii) $H^{-1}(G)$ is a finite flat group scheme, $H^{0}(G)$ is a duabelian group, and $2 \in \mathscr{O}_{S}^{\times}$. Then $G$ is dualizable.

Proof. Assume (i). By 2.16, the group $G$ fits in an extension $0 \rightarrow A \stackrel{i}{\rightarrow} G \rightarrow F \rightarrow 0$ where $A$ is an abelian scheme and $F$ is a finite flat group scheme. We have seen in the proof of 2.18 that $E^{1}(i): E^{1}(G) \rightarrow E^{1}(A)$ is an isomorphism. This implies that $H^{0}(D(G)) \rightarrow H^{0}(D(A))$ is an isomorphism (3.4). By 3.18 a) this shows that the sequence

$$
0 \longrightarrow D(F) \longrightarrow D(G) \longrightarrow D(A) \longrightarrow 0
$$

is exact. Recall that $D(F) \simeq B F^{D}$ and $D(A) \simeq A^{t}$. By 11.5 any morphism $F \rightarrow E^{2}\left(A^{t}\right)$ is trivial. Hence by 3.19 the sequence

$$
0 \longrightarrow D D(A) \longrightarrow D D(G) \longrightarrow D D(F) \longrightarrow 0
$$

is super-exact. Since $A$ and $F$ are dualizable (4.7), it follows that $G$ is dualizable as well.

Now assume (ii). By 4.5, to prove that $e_{G}$ is an isomorphism it suffices to prove that $H^{i}\left(e_{G}\right)$ is an isomorphism for $i=-1,0$. Applying 3.17 twice, we see that the stacks $D(G)$ and $D D(G)$ are algebraic and satisfy the same assumptions as $G$. Hence we can apply 3.20 and we may assume that $S$ is the spectrum of an algebraically closed field. But in this case, 4.11 applies hence $e_{G}$ is an isomorphism.

We summarize in the following table some classes of stacks which are known to be dualizable so far. For each line of this table, the 2 -functor $D($.$) induces a 2$-antiequivalence between the class on the left and the class on the right. In the last line we assume that $2 \in \mathscr{O}_{S}^{\times}$.

\begin{tabular}{|l|l|}
\hline \multicolumn{2}{|c|}{$\mathrm{D}()}$. \\
\hline Cartier group schemes (see 3.7) & classifying stacks of Cartier group schemes \\
\hline abelian schemes & abelian schemes \\
\hline 1-motives & 1-motives \\
\hline abelian stacks (see 2.15) & duabelian group schemes (see 2.17) \\
\hline $\begin{array}{l}\text { commutative group stacks } G \text { with } H^{-1}(G) \\
\text { finite flat and } H^{0}(G) \text { duabelian }\end{array}$ & $\begin{array}{l}\text { commutative group stacks } G \text { with } H^{-1}(G) \\
\text { finite flat and } H^{0}(G) \text { duabelian }\end{array}$ \\
\hline
\end{tabular}

Remark 4.14. Assume that 1.1 (1) is true. Then 1.1 (2) also holds. (Note however that $H^{-1}(D(G))$ is not flat in general.) Indeed, let $G$ be a proper, flat and finitely presented commutative group stack, with $H^{-1}(G)$ finite and flat. By 3.14 $D(G)$ is algebraic and of finite presentation. By our assumption it is even proper and flat. Hence applying 3.14 again $D D(G)$ is algebraic and of finite presentation. Since $H^{-1}(G)$ is flat, and since the result is known over an algebraically closed field (4.11), by 3.20 the morphism $H^{-1}\left(e_{G}\right)$ is an isomorphism. In particular $H^{-1}(D D(G))$ is flat. Then by Artin's theorem [39, 10.8] the coarse moduli sheaves $H^{0}(G)$ and $H^{0}(D D(G))$ are algebraic spaces. Moreover $H^{0}(G)$ is flat (because $G$ is flat, flatness is local at the source [32, IV, 2.2.11], and $G \rightarrow H^{0}(G)$ is an fppf epimorphism by [39, 10.8]) and by [3.20 again $H^{0}\left(e_{G}\right)$ is an isomorphism. Hence $e_{G}$ is an isomorphism by 4.5 .

\section{TORSORS UNDER A COMMUTATIVE GROUP STACK}

In this whole section, $G$ is a commutative group stack over a base scheme $S$. We denote by $e: S \rightarrow G$ the neutral section. The definition of a torsor under $G$ was given by Breen in 15. Note that we switched to a multiplicative notation for the "addition" of the group stack. 
Definition 5.1. (i) An action of $G$ on an $S$-stack $T$ is a pair $(\mu, \alpha)$ where $\mu: G \times_{S} T \rightarrow T$ is a morphism of $S$-stacks, and $\alpha$ is a 2-isomorphism making the following diagram 2-commutative.

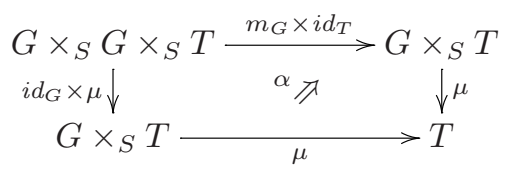

In other words, there is a functorial collection of isomorphisms

$$
\alpha_{g, h}^{x}: g \cdot(h \cdot x) \longrightarrow(g h) \cdot x
$$

for all objects $x$ of $T$ and $g, h$ of $G$, where a notation like $h . x$ stands for $\mu(h, x)$. Moreover, we require the following two conditions:

a) For all objects $x$ of $T$ and $g, h, k$ of $G$, we have a commutative diagram of 2-isomorphisms:

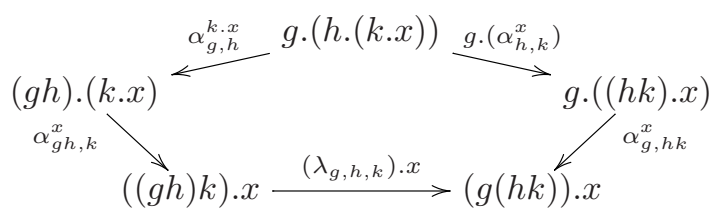

b) For any $g \in G(S)$, the translation $\mu_{g}: T \rightarrow T$ defined by $\mu_{g}(t)=g$.t is an equivalence of categories.

(ii) Let $f_{0}: G \rightarrow G^{\prime}$ be a homomorphism from $G$ to another commutative group stack $G^{\prime}$ and let $\left(T^{\prime}, \mu^{\prime}, \alpha^{\prime}\right)$ be an $S$-stack with an action of $G^{\prime}$. An $f_{0}$-equivariant morphism 1 from $T$ to $T^{\prime}$ is a pair $\left(f_{1}, \sigma\right)$ where $f_{1}: T \rightarrow T^{\prime}$ is a morphism of $S$-stacks and $\sigma$ is a 2-isomorphism making the following diagram commutative.

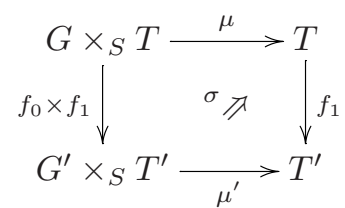

In other words, $\sigma$ is a functorial collection of isomorphisms:

$$
\sigma_{g}^{x}: f_{0}(g) \cdot f_{1}(x) \longrightarrow f_{1}(g \cdot x) .
$$

We moreover require that these isomorphisms satisfy a compatibility condition with the other data, i.e. for all objects $g, h$ in $G$ and $x$ in $T$, the following diagram of 2-isomorphisms is commutative.

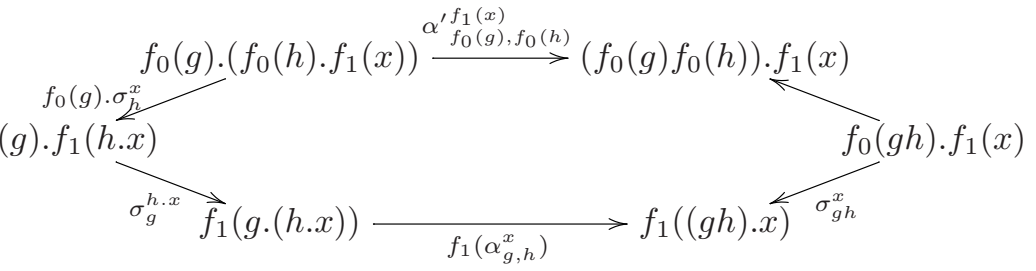

(iii) If $\left(f_{1}, \sigma\right)$ and $\left(f_{1}^{\prime}, \sigma^{\prime}\right)$ are two $f_{0}$-equivariant morphisms as in (ii), a 2-isomorphism from $\left(f_{1}, \sigma\right)$ to $\left(f_{1}^{\prime}, \sigma^{\prime}\right)$ is a 2-isomorphism $\tau: f_{1} \Rightarrow f_{1}^{\prime}$ that is compatible with the $\sigma$ 's, i.e. such that for any objects $x$ of $T$ and $g$ of $G, \tau^{g \cdot x} \circ \sigma_{g}^{x}=\sigma_{g}^{\prime x} \circ\left(f_{0}(g) \cdot \tau^{x}\right)$.

Remark 5.2. Given an $S$-stack $T$ and a pair $(\mu, \alpha)$ satisfying the pentagon condition (i) a) of 5.1 . the following requirements are equivalent (but not automatic, e.g. if $\mu$ is a constant morphism then it satisfies (i) a) but not (i) b)):

(i) b) For any $g \in G(S)$, the translation $\mu_{g}$ is an equivalence of categories.

(i) b') For some $g \in G(S)$, the translation $\mu_{g}$ is an equivalence of categories.

\footnotetext{
${ }^{1}$ If $G=G^{\prime}$ and $f_{0}=\operatorname{id}_{G}$ we will talk about a $G$-equivariant morphism.
} 
(i) c) For some neutral object $(e, \varepsilon)$, there is a (automatically unique) 2-isomorphism

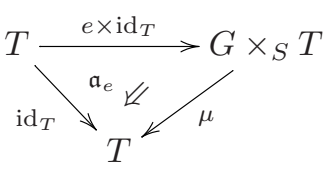

in other words a functorial collection of isomorphisms $\mathfrak{a}_{e}^{x}: e . x \rightarrow x$, and for all objects $g$ of $G$ and $x$ of $T$ the following diagrams of 2-isomorphisms commute:
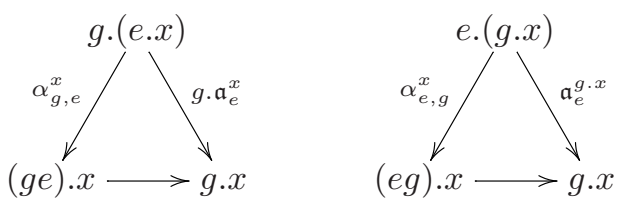

where the bottom maps are uniquely determined by $\varepsilon$.

(i) c') For any neutral object $(e, \varepsilon)$ there is a (unique) 2-isomorphism $\mathfrak{a}_{e}$ as in (i) c).

Remark 5.3. Let $\left(f_{1}, \sigma\right)$ be an $f_{0}$-equivariant morphism as in (ii). Let $(e, \varepsilon)$ be a neutral object of $G$. Its image $e^{\prime}=f_{0}(e)$ is a neutral object of $G^{\prime}$. Let $\mathfrak{a}_{e}$ and $\mathfrak{b}_{e^{\prime}}$ be the associated 2-isomorphisms as in (i) c) above. Then for any $x$ in $T$, the following diagram commutes:

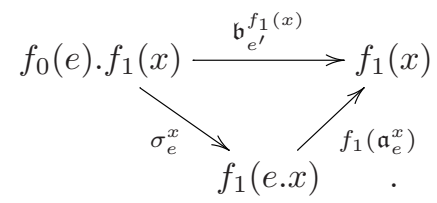

The following lemma is straightforward.

Lemma 5.4. Let $T$ be an $S$-stack, with an action $(\mu, \alpha)$ of $G$. The following are equivalent:

(i) Fppf-locally on $S$, there is a G-equivariant isomorphism $f_{1}: G \rightarrow T$.

(ii) The natural morphism $\left(\mu, p_{2}\right): G \times{ }_{S} T \rightarrow T \times{ }_{S} T,(g, t) \mapsto(g . t, t)$ is an equivalence, and the morphism $T \rightarrow S$ is an fppf epimorphism, i.e. there is an fppf covering $S^{\prime} \rightarrow S$ such that $T\left(S^{\prime}\right)$ is nonempty.

Definition 5.5. A $G$-torsor is an $S$-stack $T$ with an action of $G$ satisfying the conditions of 5.4 .

For any $G$-torsor $T$, and any stack $P$ with an action of $G$, Breen defines in [15] a contracted product $T \wedge^{G} P$ that inherits a natural action of $G$. Let us recall some properties of this construction.

Proposition 5.6. a) If $T_{1}$ and $T_{2}$ are two $G$-torsors, then $T_{1} \wedge^{G} T_{2}$ is again a G-torsor. This defines a group law on the set $H^{1}(S, G)$ of isomorphism classes of $G$-torsors, where the neutral element is the class of the trivial torsor $G$.

b) Let $T$ be a G-torsor and $\varphi: G \rightarrow H$ a morphism of commutative group stacks. This induces a natural $G$-action on $H$. Then $H$ naturally acts on the stack $T \wedge^{G} H$ and makes it an $H$-torsor (which we denote by $T \wedge^{G, \varphi} H$ if there is an ambiguity on the morphism $\varphi)$. This defines a group morphism $H^{1}(\varphi): H^{1}(S, G) \rightarrow H^{1}(S, H)$.

Proposition 5.7. Let $G$ and $H$ be two commutative group stacks over a base scheme $S$ and let $\varphi_{1}$ and $\varphi_{2}$ be two homomorphisms of commutative group stacks from $G$ to $H$. Let $\psi$ denote their product, defined functorially by $\psi(g)=\varphi_{1}(g) \varphi_{2}(g)$. Then for a $G$-torsor $T$, there is a functorial isomorphism

$$
T \wedge^{G, \psi} H \simeq\left(T \wedge^{G, \varphi_{1}} H\right) \wedge^{H}\left(T \wedge^{G, \varphi_{2}} H\right) .
$$

We can also describe $G$-torsors in terms of extensions of $\mathbb{Z}$ by $G$. We define a 2-category $\operatorname{Ext}^{1}(\mathbb{Z}, G)$ as follows.

(1) An object is an exact sequence of commutative group stacks $0 \rightarrow G \stackrel{j}{\rightarrow} E \stackrel{\pi}{\rightarrow} \mathbb{Z} \rightarrow 0$.

(2) A morphism between two such objects $\left(E_{1}, j_{1}, \pi_{1}\right)$ and $\left(E_{2}, j_{2}, \pi_{2}\right)$ is a pair $(\varphi, \beta)$ where $\varphi: E_{1} \rightarrow E_{2}$ is a homomorphism of commutative group stacks such that $\pi_{2} \circ \varphi=\pi_{1}$ and $\beta: \varphi \circ j_{1} \Rightarrow j_{2}$ is a 2-isomorphism of additive morphisms (see 2.4). 
(3) A 2 -isomorphism from $(\varphi, \beta)$ to $\left(\varphi^{\prime}, \beta^{\prime}\right)$ is a 2 -isomorphism $\delta: \varphi \Rightarrow \varphi^{\prime}$ of additive morphisms (see 2.4), that is compatible with $\beta$ and $\beta^{\prime}$.

If $0 \rightarrow G \stackrel{j}{\rightarrow} E \stackrel{\pi}{\rightarrow} \mathbb{Z} \rightarrow 0$ is an object of $E x t^{1}(\mathbb{Z}, G)$, then $\pi^{-1}(1)$ is naturally a $G$-torsor. This construction extends to a 2 -functor $t$ from $\operatorname{Ext}^{1}(\mathbb{Z}, G)$ to $(G-$ Tors $)$. We leave to the reader the proof of the following fact.

Proposition 5.8. The 2-functort from $\operatorname{Ext}^{1}(\mathbb{Z}, G)$ to $(G$-Tors $)$ is a 2-equivalence of 2-categories.

To conclude this section, we recall a few facts about $F$-gerbes for a fixed sheaf of commutative groups $F$, in particular the equivalence between the notions of an $F$-gerbe and a $B F$-torsor.

Definition 5.9. $\quad$ (i) Let $f: X \rightarrow S$ be a stack over an algebraic space $S$. We say that $f$ is a gerbe (or that $X$ is a gerbe over $S$ ) if $f$ and its diagonal are fppf epimorphisms.

(ii) Let $F$ be a sheaf of commutative groups over $S$. An F-gerbe, or a gerbe banded by $F$, is a gerbe $f: X \rightarrow S$ together with an isomorphism of sheaves of groups

$$
c_{x}: F_{U} \longrightarrow \operatorname{Aut}_{X}(x)
$$

for every $S$-scheme $U$ and every object $x \in X(U)$, such that the following conditions hold: (G1) For any $S$-scheme $U$ and any isomorphism $\varphi: x \rightarrow x^{\prime}$ in $X(U)$, we have $c_{\varphi} \circ c_{x}=c_{x^{\prime}}$ where $c_{\varphi}: \operatorname{Aut}_{X}(x) \rightarrow \operatorname{Aut}_{X}\left(x^{\prime}\right)$ is the isomorphism induced by $\varphi$.

(G2) For any $S$-morphism $V \rightarrow U$ and any object $x \in X(U)$, the pullback of $c_{x}$ along $V \rightarrow U$ is equal to $c_{\left(x_{\mid}\right)}$.

(iii) A morphism of F-gerbes from $\left(X^{\prime},\left\{c_{x^{\prime}}^{\prime}\right\}\right)$ to $\left(X,\left\{c_{x}\right\}\right)$ is a morphism of stacks $g: X^{\prime} \rightarrow X$ such that for every object $x^{\prime}$ of $X^{\prime}$ the diagram

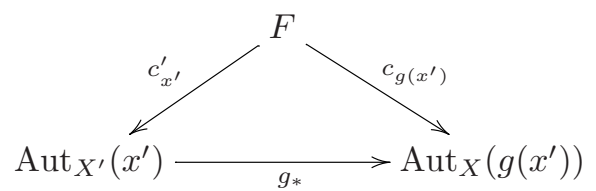

commutes.

(iv) If $g$ and $h$ are two morphisms of F-gerbes, a 2-isomorphism from $g$ to $h$ is a 2-isomorphism $g \Rightarrow h$ of morphisms of stacks.

Remark 5.10. Equivalently, an $F$-gerbe is a gerbe $X \rightarrow S$ together with an isomorphism of sheaves of groups over $X$ :

$$
c: F_{X} \longrightarrow I_{X / S}
$$

where $F_{X}=F \times_{S} X$ and $I_{X / S}$ is the inertia stack of $X$ over $S$.

We refer to 31 or 43 for some elementary facts about gerbes banded by a sheaf of commutative groups $F$. In particular there is a natural bijection ([31, IV, 3.4.2] or [43, 12.2.4])

$$
\gamma_{F}: H^{2}(S, F) \longrightarrow\{\text { isomorphism classes of } F-\text { gerbes }\}
$$

where the group on the left is the fppf derived functor cohomology group. The result below is folklore, but we were unable to find a suitable reference.

Proposition 5.11. (1) There is a natural 2-equivalence of 2-categories between the 2-category of BF-torsors and the 2-category of F-gerbes.

(2) If the set of isomorphism classes of F-gerbes is equipped via this equivalence with the group law induced by the contracted product of BF-torsors from 5.6, then the above bijection $\gamma_{F}$ is a group isomorphism.

Proof. Since we did not even describe $\gamma_{F}$, we only give the proof of (1). Let us construct a 2equivalence $\Phi$ from $B F$-torsors to $F$-gerbes. Let $T$ be a $B F$-torsor. By definition $T \rightarrow S$ is an fppf epimorphism. Moreover since $T$ is locally isomorphic to $B F$, and since two $F$-torsors are locally isomorphic (because they are both locally trivial), the diagonal of $T \rightarrow S$ is also an fppf epimorphism. Hence $T \rightarrow S$ is a gerbe. Let $t$ be an object of $T(U)$ for some $S$-scheme $U$. Then the morphism $\mu_{t}: g \mapsto g . t$ is an isomorphism of stacks $(B F)_{U} \rightarrow T_{U}$, which induces an isomorphism of group sheaves $\operatorname{Aut}_{(B F)_{U}}(e) \simeq \operatorname{Aut}_{T_{U}}(t)$ where $e$ denotes the neutral object of $B F$, 
i.e. the trivial $F$-torsor. Composing with the canonical isomorphism $\operatorname{Aut}_{B F}(e) \simeq F$ we get an isomorphism of group sheaves over $U$

$$
c_{t}: F_{U} \longrightarrow \operatorname{Aut}_{T}(t) .
$$

Now the stack $T$ together with the collection of all the $c_{t}$ 's is an $F$-gerbe. This defines $\Phi$ on objects. The definition of $\Phi$ on 1-morphisms and on 2-morphisms is straightforward: if $\left(f: T_{1} \rightarrow T_{2}, \sigma\right)$ is a morphism of $B F$-torsors then $\Phi(f, \sigma)$ is just $f$. Using the functoriality of $\sigma$ and Remark 5.3 we check that the diagrams of 5.9 (iii) commute. If $\tau: f \Rightarrow f^{\prime}$ is a 2-isomorphism from $(f, \sigma)$ to $\left(f^{\prime}, \sigma^{\prime}\right)$ we define $\Phi(\tau)=\tau$.

It remains to prove that $\Phi$ is indeed a 2 -equivalence of categories, i.e. it is 2-essentially surjective, and for any two $B F$-torsors $T_{1}$ and $T_{2}$, the functor $\Phi_{T_{1}, T_{2}}: \operatorname{Hom}\left(T_{1}, T_{2}\right) \rightarrow \operatorname{Hom}\left(\Phi\left(T_{1}\right), \Phi\left(T_{2}\right)\right)$ is an equivalence. Let us first prove that $\Phi_{T_{1}, T_{2}}$ is fully faithfull. This amounts to say that if $(f, \sigma)$ and $\left(f^{\prime}, \sigma^{\prime}\right)$ are two morphisms of $B F$-torsors, then any 2-isomorphism $\tau: f \Rightarrow f^{\prime}$ is compatible with $\sigma$ and $\sigma^{\prime}$ in the sense of 5.1 (iii), i.e. for any objects $g$ of $B F$ and $x$ of $T_{1}$, we have $\tau^{g \cdot x} \circ \sigma_{g}^{x}=\sigma_{g}^{\prime x} \circ\left(g \cdot \tau^{x}\right)$. Using the fact that we have a functorial identification of the automorphism groups of the objects of $T_{2}$ with $F$, we can define a morphism of stacks $B F \times{ }_{S} T_{1} \rightarrow F$ that maps a pair $(g, x)$ to the section of $F$ that corresponds to $\tau^{g \cdot x} \circ \sigma_{g}^{x} \circ\left(\sigma_{g}^{\prime x} \circ\left(g \cdot \tau^{x}\right)\right)^{-1}$. Now the key fact is that the coarse moduli space of $B F \times{ }_{S} T_{1}$ is $S$, so that this morphism factorizes through $S$, by the universal property of the coarse moduli space. It then suffices to check the required equality when $g=e$ (the neutral object of $B F$ ), in which case this boils down to the functoriality of $\tau$ and Remark 5.3 ,

Let us prove that $\Phi_{T_{1}, T_{2}}$ is essentially surjective. Let $f: T_{1} \rightarrow T_{2}$ be a morphism of stacks that satisfies the condition 5.9 (iii). We have to prove that there exists a 2-isomorphism $\sigma$ such that $(f, \sigma)$ is a morphism of $B F$-torsors. For this, we recall the fact that if $T$ is an $F$-gerbe, then for any object $x$ of $T$, we have an isomorphism of $F$-gerbes:

$$
\operatorname{Triv}_{x}: T \longrightarrow B F
$$

that maps an object $y$ of $T$ to the sheaf $\operatorname{Isom}(y, x)$. Moreover, if $T$ is a $B F$-torsor and $x$ is an object of $T$, then $\operatorname{Triv}_{x}: T \rightarrow B F$ is a quasi-inverse to the equivalence $\mu_{x}: B F \rightarrow T$. [This amounts to say that any $F$-torsor $g$ is isomorphic to $\operatorname{Triv}_{x}\left(\mu_{x}(g)\right)$, i.e. to $\operatorname{Isom}(g \cdot x, x)$. To see this, notice that a section of $g$ corresponds to a trivialization $g \simeq e$ where $e$ is the trivial $F$-torsor, hence to an isomorphism $g . x \simeq x$, via $\mu_{x}$ and the canonical isomorphism $e . x \simeq x$.] For a fixed object $x$ of $T_{1}$, the following diagram is 2 -commutative

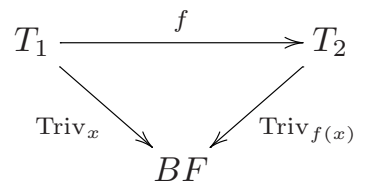

where the 2-isomorphism is given by the collection of the isomorphisms of $F$-torsors

$$
\operatorname{Isom}(y, x) \rightarrow \operatorname{Isom}(f(y), f(x))
$$

induced by $f$. It follows that the diagram

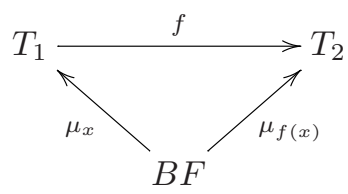

is 2-commutative as well. Now the 2-isomorphism $\mu_{f(x)} \Rightarrow f \circ \mu_{x}$ yields the desired functorial collection of isomorphisms $\sigma_{g}^{x}: g . f(x) \rightarrow f(g \cdot x)$.

Finally let us prove that $\Phi$ is 2-essentially surjective. Let $\left(T,\left\{c_{t}\right\}\right)$ be an $F$-gerbe. We have to construct a morphism $\mu: B F \times{ }_{S} T \rightarrow T$ together with a 2-isomorphism $\alpha$ that satisfies the conditions of 5.1 such that $\Phi(T, \mu, \alpha) \simeq\left(T,\left\{c_{t}\right\}\right)$. Let $(g, t)$ be a point of $B F \times_{S} T$. Recall that $\operatorname{Triv}_{t}: T \rightarrow B F$ is an equivalence and choose a quasi-inverse $\mu_{t}: B F \rightarrow T$. We define $\mu(g, t)$ to be $\mu_{t}(g)$. The remaining details (constructing $\alpha$ and checking that $\left.\Phi(T, \mu, \alpha) \simeq\left(T,\left\{c_{t}\right\}\right)\right)$ are left to the reader. 


\section{A THEOREM OF THE SQUARE}

For an abelian variety $A$ over a field $k$, the classical "theorem of the square" asserts that for all $x, y \in A(k)$, and for any line bundle $L$ on $A$, there is an isomorphism $\left(\mu_{x+y}^{*} L\right) \otimes L \simeq\left(\mu_{x}^{*} L\right) \otimes\left(\mu_{y}^{*} L\right)$, where for a point $x$ of $A$, the morphism $\mu_{x}: A \rightarrow A$ is the translation $a \mapsto a+x$. We will need similar facts for some commutative group stacks: abelian stacks on the one hand, and classifying stacks on the other hand. This section is a short interlude devoted to the proof of these facts.

Definition 6.1. Let $G$ be a commutative group stack over a base scheme $S$. Let $L$ be an invertible sheaf on $G$. We denote by $\Lambda(L)$ the so-called "Mumford bundle" on $G \times{ }_{S} G$

$$
\Lambda(L)=\left(\mu^{*} L\right) \otimes\left(p_{1}^{*} L\right)^{-1} \otimes\left(p_{2}^{*} L\right)^{-1}
$$

where $\mu$ is the product map and $p_{1}, p_{2}$ are the projections from $G \times{ }_{S} G$ to $G$. We denote by $\varphi_{L}$ the induced morphism of stacks:

$$
\varphi_{L}: G \longrightarrow \mathrm{Pic}_{G / S} \cdot
$$

Functorially, $\varphi_{L}$ maps a point $g \in G(S)$ to the class $\left[\mu_{g}^{*} L \otimes L^{-1}\right]$.

Theorem 6.2. Let $G$ be a commutative group stack over a base scheme $S$ and let $L$ be an invertible sheaf on $G$. Assume that one of the following holds:

(a) $G$ is an abelian stack and $[L] \in \operatorname{Pic}_{G / S}^{\tau}(S)$.

(b) $G$ is the classifying stack $B F$ of a commutative group scheme $F$. Then $\varphi_{L}=0$.

Proof. By the universal property of the coarse moduli space [39, (3.19)], $\varphi_{L}$ factorizes through the coarse moduli space of $G$. In the case (b), this moduli space is trivial, hence $\varphi_{L}$ is constant, and equal to 0 since $\varphi_{L}(e)=0$.

In the case (a), let us denote by $A=H^{0}(G)$ and $F=H^{-1}(G)$. Then there is an exact sequence of commutative group stacks:

$$
0 \longrightarrow \text { BF } \stackrel{i}{\longrightarrow} G \stackrel{\pi}{\longrightarrow} A \longrightarrow 0 .
$$

By assumption, the group $F$ is finite and flat over $S$ and $A$ is an abelian scheme over $S$. We may assume that $F$ is locally free of rank $n$, so that $n F^{D}=0$. By 3.24 there is an exact sequence of group schemes:

$$
0 \longrightarrow \operatorname{Pic}_{A / S} \stackrel{\pi^{*}}{\longrightarrow} \operatorname{Pic}_{G / S} \stackrel{\chi}{\longrightarrow} F^{D} .
$$

Let us first assume that there is an invertible sheaf $M$ on $A$ such that $L \simeq \pi^{*} M$. Since $[L] \in$ $\operatorname{Pic}_{G / S}^{\tau}$ there exists an integer $m>0$ such that $[L]^{m} \in \operatorname{Pic}_{G / S}^{0}$. The morphism $x \mapsto x^{n}$ from Pic ${ }_{G / S}^{0}$ to itself factorizes through $\mathrm{Pic}_{A / S}$ because of the above exact sequence, hence through $\mathrm{Pic}_{A / S}^{0}$ because $\operatorname{Pic}_{G / S}^{0}$ has connected fibers. This proves that $[M]^{n m} \in \operatorname{Pic}_{A / S}^{0}$ hence $[M] \in \operatorname{Pic}_{A / S}^{\tau}$. Using the theorem for the abelian scheme $A$ (see [42, chap. 6 §2]) we see that for any object $g$ of $G$, the class of $\mu_{\pi(g)}^{*} M \otimes M^{-1}$ is trivial in $\operatorname{Pic}_{A / S}$, hence its pullback $\left[\mu_{g}^{*} L \otimes L^{-1}\right]$ is trivial in $\mathrm{Pic}_{G / S}$ and this proves the theorem in this case.

In the general case, by 3.21, the composition $\chi \circ \varphi_{L}$ from $G$ to $F^{D}$ must be constant, hence trivial since $\varphi_{L}(e)=0$. This proves that $\varphi_{L}$ factorizes through $\mathrm{Pic}_{A / S}$, and even through $A^{t}=$ $\operatorname{Pic}_{A / S}^{0}$ since $G$ has geometrically connected fibers. Let us still denote by $\varphi_{L}$ the induced morphism $G \rightarrow A^{t}$. Then $\chi\left(L^{n}\right)=0$ in $F^{D}(S)$ and it follows that $L^{n}$ comes from $A$. By the previous case we deduce that $\varphi_{L^{n}}=0$. But $\varphi_{L^{n}}$ is equal to $\left(\varphi_{L}\right)^{n}$ so $\varphi_{L}$ factorizes through the kernel $A_{n}^{t}$ of the isogeny $[n]: A^{t} \rightarrow A^{t}$. Since $A_{n}^{t}$ is finite, using 3.21 again we deduce that $\varphi_{L}$ is constant equal to 0 .

Remark 6.3. In the case (b), we can give a more precise statement. Let $L$ be an invertible sheaf on $G$ and let $x \in G(S)$. Let us denote by $\chi_{L}: F \rightarrow \mathbb{G}_{\mathrm{m}}$ the character of $L$ and by $T_{x}$ the $F$-torsor corresponding to the point $x: S \rightarrow B F$. Then we can prove that the line bundle $\mu_{x}^{*} L \otimes L^{-1}$ is isomorphic to $\pi^{*} \mathscr{L}\left(\chi_{L}, T_{x}\right)$ where $\pi: G \rightarrow S$ is the structural morphism of $G$ and $\mathscr{L}\left(\chi_{L}, T_{x}\right)$ is the line bundle on $S$ corresponding to the $\mathbb{G}_{\mathrm{m}}$-torsor $T_{x} \wedge^{F, \chi_{L}} \mathbb{G}_{\mathrm{m}}$. 
Corollary 6.4. Let $G$ be an abelian stack (resp. the classifying stack BF of a commutative group scheme $F$ ).

(1) For any $x \in G(S)$, the translation $\mu_{x}: G \rightarrow G$ induces the identity on Pic $_{G / S}^{\tau}$ (resp. on $\left.\mathrm{Pic}_{G / S}\right)$.

(2) Let $x, y \in G(S)$. There is a functorial collection of isomorphisms

$$
\delta_{L}:\left(\mu_{x+y}^{*} L\right) \otimes L \stackrel{\sim}{\longrightarrow}\left(\mu_{x}^{*} L\right) \otimes\left(\mu_{y}^{*} L\right)
$$

for all line bundles $L$ on $G$ such that $[L] \in \operatorname{Pic}_{G / S}^{\tau}(S)$ (resp. for all line bundles $L$ on $G$ ).

Proof. (1) is a reformulation of 6.2 Let us prove (2). Let $x, y \in G(S)$ and let $L$ be a line bundle on $G$ such that $\varphi_{L}=0$. Then $\left[\mu_{x}^{*} L \otimes L^{-1}\right]=0$ hence there is a line bundle $N$ on $S$ with an isomorphism $\psi: \mu_{x}^{*} L \otimes L^{-1} \rightarrow \pi^{*} N$, where $\pi: G \rightarrow S$ is the structural morphism. Since $\pi \mu_{y}=\pi$, there is a canonical isomorphism

$$
c(N): \mu_{y}^{*} \pi^{*} N \longrightarrow \pi^{*} N .
$$

We deduce an isomorphism $\gamma(L):=\psi^{-1} c(N) \mu_{y}^{*}(\psi)$ from $\mu_{y}^{*}\left(\mu_{x}^{*} L \otimes L^{-1}\right)$ to $\mu_{x}^{*} L \otimes L^{-1}$. This isomorphism does not depend on the choice of $N$ or $\psi$ since $c(N)$ is functorial. Moreover it is functorial in L. Via the canonical isomorphism $\mu_{y}^{*} \mu_{x}^{*} \simeq \mu_{x+y}^{*}$, it induces the desired $\delta_{L}$.

\section{The Albanese torsor}

Let $X$ be an algebraic stack over a base scheme $S$ and denote by $f: X \rightarrow S$ its structural morphism. Assume that $\mathscr{O}_{S} \rightarrow f_{*} \mathscr{O}_{X}$ is universally an isomorphism, i.e. for any morphism $T \rightarrow S$, the morphism $\mathscr{O}_{T} \rightarrow\left(f_{T}\right)_{*} \mathscr{O}_{X_{T}}$ is an isomorphism, where $f_{T}: X_{T} \rightarrow T$ is the morphism obtained from $f$ after the base change $T \rightarrow S$. Assume also that $f$ locally has sections in the fppf topology. Then we have an exact sequence of group stacks (see [17, 2.3]):

$$
0 \longrightarrow B \mathbb{G}_{\mathrm{m}} \longrightarrow \operatorname{P} i c(X / S) \longrightarrow \operatorname{Pic}_{X / S} \longrightarrow 0 \text {. }
$$

Since $f$ locally has sections, so does $D\left(f^{*}\right): D(\mathscr{P} i c(X / S)) \rightarrow D\left(B \mathbb{G}_{\mathrm{m}}\right)$, hence $D\left(f^{*}\right)$ is an fppf epimorphism. By $3.18 \mathrm{a})$, the dual sequence is exact:

$$
0 \longrightarrow D\left(\operatorname{Pic}_{X / S}\right) \stackrel{j}{\longrightarrow} D(\mathscr{P} i c(X / S)) \stackrel{\pi}{\longrightarrow} \mathbb{Z} \longrightarrow 0 \text {. }
$$

By 5.8 this sequence corresponds to a $D\left(\operatorname{Pic}_{X / S}\right)$-torsor over $S$.

Definition 7.1. $\quad$ (i) The Albanese stack of $X$ is the commutative group stack

$$
A^{0}(X):=D\left(\operatorname{Pic}_{X / S}\right) .
$$

(ii) The Albanese torsor of $X$ is the $A^{0}(X)$-torsor corresponding to the above sequence via Proposition 5.8. It is denoted by $A^{1}(X)$.

If $x$ is an object of $X(U)$ for some $S$-scheme $U$, we still denote by $x$ the induced section $U \rightarrow X_{U}:=X \times_{S} U$. Then the pullback $x^{*}$ defines a morphism of commutative group stacks from $\mathscr{P}_{i c}\left(X_{U} / U\right)$ to $\mathscr{P} i c(U / U) \simeq\left(B \mathbb{G}_{\mathrm{m}}\right)_{U}$. This is functorial, hence this defines a natural morphism of stacks $\varphi: X \rightarrow D(\mathscr{P} i c(X / S))$. Let us compute the composition $\pi \circ \varphi: X \rightarrow \mathbb{Z}$. Let $x: S \rightarrow X$ be an $S$-point of $X$. If we identify $\mathscr{P} i c(X / S)$ with the stack $\operatorname{Mor}\left(X, B \mathbb{G}_{\mathrm{m}}\right)$ of morphisms from $X$ to $B \mathbb{G}_{\mathrm{m}}$ (and $B \mathbb{G}_{\mathrm{m}}$ with $\operatorname{Mor}\left(S, B \mathbb{G}_{\mathrm{m}}\right)$ ), then $\varphi(x)$ is the "evaluation at $x "$ morphism $e v_{x}: \operatorname{Mor}\left(X, B \mathbb{G}_{\mathrm{m}}\right) \rightarrow B \mathbb{G}_{\mathrm{m}}$ defined functorially by $\tau \mapsto \tau \circ x$. Under the identification of $\operatorname{Hom}\left(B \mathbb{G}_{\mathrm{m}}, B \mathbb{G}_{\mathrm{m}}\right)$ with $\mathbb{Z}$, by definition $\pi$ is the dual of the canonical morphism $f^{*}: B \mathbb{G}_{\mathrm{m}} \rightarrow \mathscr{P} i c(X / S)$, hence $\pi(\varphi(x))$ is the section of $\mathbb{Z}$ that corresponds to the morphism $e v_{x} \circ f^{*}: B \mathbb{G}_{\mathrm{m}} \rightarrow B \mathbb{G}_{\mathrm{m}}$. The latter morphism maps an $S$-point $t: S \rightarrow B \mathbb{G}_{\mathrm{m}}$ of $B \mathbb{G}_{\mathrm{m}}$ to the composite $t \circ f \circ x$. But $f \circ x=\mathrm{id}_{S}$, so that $e v_{x} \circ f^{*}$ is the identity of $B \mathbb{G}_{\mathrm{m}}$, which corresponds to the section $1 \in \mathbb{Z}$. Hence $\pi \circ \varphi$ is constant equal to 1 . This means that $\varphi$ factorizes through the open and closed substack $A^{1}(X)$ of $D(\mathscr{P} i c(X / S))$.

Definition 7.2. The induced morphism from $X$ to $A^{1}(X)$ is called the Albanese morphism of $X$ and is denoted by

$$
a_{X}: X \longrightarrow A^{1}(X) \text {. }
$$


Remark 7.3. Note that the Albanese morphism is functorial. Let $g: X \rightarrow Y$ be a morphism between algebraic stacks satisfying the above assumptions. Let us denote by $A^{0}(g)$ the dual of $g^{*}: \operatorname{Pic}_{Y / S} \rightarrow \operatorname{Pic}_{X / S}$. Then the morphism $D\left(g^{*}\right)$ from $D(\mathscr{P} i c(X / S))$ to $D(\mathscr{P} i c(Y / S))$ induces an $A^{0}(g)$-equivariant morphism of torsors $A^{1}(g): A^{1}(X) \rightarrow A^{1}(Y)$. Moreover, there is a canonical 2-isomorphism making the natural square

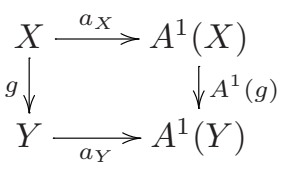

2-commutative.

Remark 7.4. Note that, for any commutative group stack $G$, the diagram

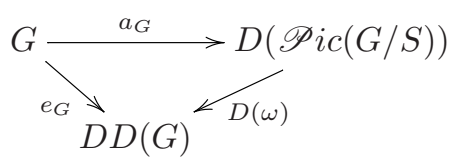

is 2-commutative. Indeed, if $g$ is an $S$-point of $G$, then $D(\omega)\left(a_{G}(g)\right)=a_{G}(g) \circ \omega=g^{*} \circ \omega$ maps an element $\psi \in D(G)$ to the $S$-point $g^{*}(\psi): S \stackrel{g}{\rightarrow} G \stackrel{\psi}{\rightarrow} \mathrm{B} \mathbb{G}_{\mathrm{m}}$ of $\mathrm{B} \mathbb{G}_{\mathrm{m}}$. The latter is equal to $\psi(g)$, hence $D(\omega) \circ a_{G}=e_{G}$. In the above diagram, be careful that $a_{G}$ is not a homomorphism of commutative group stacks (it does not even map 0 to 0 ).

Remark 7.5. For any morphism of commutative group stacks $A \rightarrow \mathrm{Pic}_{X / S}$, we can extend the scalars along the dual morphism $A^{0}(X) \rightarrow D(A)$ and get a morphism $X \rightarrow A^{1}(X) \wedge^{A^{0}(X)} D(A)$ from $X$ to a $D(A)$-torsor. In particular, we will denote as follows the duals of the neutral and torsion components:

$$
A_{0}^{0}(X):=D\left(\operatorname{Pic}_{X / S}^{0}\right), A_{\tau}^{0}(X):=D\left(\operatorname{Pic}_{X / S}^{\tau}\right)
$$

and the resulting torsors will be denoted by $A_{0}^{1}(X)$ and $A_{\tau}^{1}(X)$ and will also be called Albanese torsor if no confusion can arise. We have natural morphisms

$$
X \longrightarrow A^{1}(X) \longrightarrow A_{\tau}^{1}(X) \longrightarrow A_{0}^{1}(X) .
$$

Note that the torsor $A_{0}^{1}(X)$ (and similarly for $A_{\tau}^{1}(X)$ ) corresponds via 5.8 to the exact sequence

$$
0 \longrightarrow D\left(\operatorname{Pic}_{X / S}^{0}\right) \longrightarrow D\left(\mathscr{P} i c^{0}(X / S)\right) \longrightarrow \mathbb{Z} \longrightarrow 0 .
$$

We prove below (17.7) that if $G$ is an abelian stack, or the classifying stack of a multiplicative group, then any $G$-torsor is the Albanese torsor of some stack (actually, it is the Albanese torsor of itself).

Proposition 7.6. Let $G$ be a commutative group stack and let $T$ be a $G$-torsor.

(a) If $G$ is an abelian stack, then there is a canonical isomorphism

$$
\operatorname{Pic}_{T / S}^{\tau} \stackrel{\sim}{\longrightarrow} \operatorname{Pic}_{G / S}^{\tau} \text {. }
$$

(b) If $G$ is the classifying stack of a commutative group scheme, then there is a canonical isomorphism

$$
\mathrm{Pic}_{T / S} \stackrel{\sim}{\longrightarrow} \operatorname{Pic}_{G / S} .
$$

(c) The isomorphisms of (a) and (b) are functorial in the following sense. Let $c_{0}: G \rightarrow G^{\prime}$ be a morphism of abelian stacks and let $c_{1}: T \rightarrow T^{\prime}$ be a c co-equivariant morphism of torsors. Then the diagram

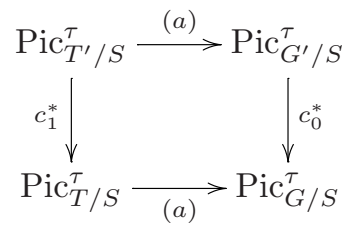


commutes (and analogue statement for (b)).

Proof. Let $t_{0} \in T\left(S^{\prime}\right)$ be an $S^{\prime}$-point of $T$ where $S^{\prime} \rightarrow S$ is an fppf cover. Such a point gives rise to an isomorphism of stacks $\varphi_{t_{0}}: G_{S^{\prime}} \rightarrow T_{S^{\prime}}$ mapping $g$ to $g . t_{0}$, which in turn induces an isomorphism $\varphi_{t_{0}}^{*}: \operatorname{Pic}_{T_{S^{\prime}} / S^{\prime}}^{\tau} \rightarrow \operatorname{Pic}_{G_{S^{\prime}} / S^{\prime}}^{\tau}$. By Corollary 6.4 the translation $\mu_{x}: G \rightarrow G$ by an $S$-point $x$ of $G$ induces the identity on $\operatorname{Pic}_{G / S}^{\tau}$. This proves that $\varphi_{t_{0}}^{*}$ does not depend on the choice of $t_{0}$. By descent this yields the isomorphism (a). Similarly we get (b). Let us prove (c). The statement is fppf-local on $S$, so we may assume that $T$ has an $S$-point $t_{0}$. Then we have to prove that $c_{0}^{*} \circ\left(\varphi_{c_{1}\left(t_{0}\right)}^{*}\right)=\varphi_{t_{0}}^{*} \circ c_{1}^{*}$. But $c_{1}$ is equivariant hence $c_{1} \circ \varphi_{t_{0}}=\varphi_{c_{1}\left(t_{0}\right)} \circ c_{0}$ and the result follows.

Proposition 7.7. Let $G$ be a commutative group stack over $S$ and let $T$ be a $G$-torsor.

(a) Assume that $G$ is an abelian stack. Let us denote by $f_{0}$ the following composition of isomorphisms:

$$
f_{0}: G \stackrel{e_{G}}{\longrightarrow} D D(G) \stackrel{D \stackrel{D .23}{\longrightarrow}}{\longrightarrow} D\left(\operatorname{Pic}_{G / S}^{\tau}\right) \stackrel{D}{\longrightarrow} D\left(\operatorname{Pic}_{T / S}^{\tau}\right)=A_{\tau}^{0}(T) .
$$

Then the canonical morphism

$$
a_{T}: T \longrightarrow A_{\tau}^{1}(T)
$$

is an $f_{0}$-equivariant isomorphism of torsors.

(b) Assume that $G$ is the classifying stack of a Cartier group scheme. Let us denote by $f_{0}$ the following composition of isomorphisms:

$$
f_{0}: G \stackrel{e_{G}}{\longrightarrow} D D(G) \stackrel{D \stackrel{13.26}{\longrightarrow}}{\longrightarrow} D\left(\mathrm{Pic}_{G / S}\right) \stackrel{D \underline{7.6}}{\longrightarrow} D\left(\mathrm{Pic}_{T / S}\right)=A^{0}(T) .
$$

Then the canonical morphism

$$
a_{T}: T \longrightarrow A^{1}(T)
$$

is an $f_{0}$-equivariant isomorphism of torsors.

Remark 7.8. In the case $T=G$, it follows from 7.4 that the isomorphism $f_{0}$ in (a) (resp. (b)) coincides with the composition $G \stackrel{a_{G}}{\longrightarrow} A_{\tau}^{1}(G) \stackrel{t_{-e^{*}}}{\longrightarrow} A_{\tau}^{0}(G)$ (resp. with $G \stackrel{a_{G}}{\longrightarrow} A^{1}(G) \stackrel{t_{-e^{*}}}{\longrightarrow} A^{0}(G)$ ) where $e$ is a neutral object of $G$ and $t_{-e^{*}}$ maps a point $\lambda$ to $\lambda-e^{*}$.

Proof. We prove (a) only, (b) is very similar. Since a morphism of torsors is always an isomorphism, it suffices to prove that $a_{T}$ is $f_{0}$-equivariant. Let us first do it locally on $S$. Then we can assume that $T$ is the group $G$ with its action by translations. We have to find a 2 -isomorphism $\alpha$ making the diagram

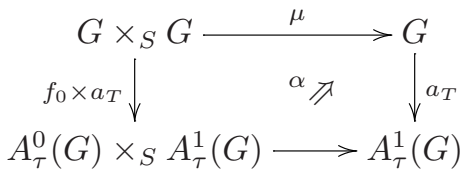

2-commutative. For any two objects $x, y$ of $G(S)$, we need a functorial isomorphism between $a_{T}(x+y)$ and $f_{0}(x) \cdot a_{T}(y)$. Both are objects of $\mathscr{H}$ om $\left(\mathscr{P} i c^{\tau}(G / S), \mathrm{B} \mathbb{G}_{\mathrm{m}}\right)$. Let us describe them. Identifying $B \mathbb{G}_{\mathrm{m}}$ with the Picard stack of $S$ over itself, we recall that, by definition, $a_{T}(y)$ is the pullback functor $y^{*}$, that is, the morphism from $\mathscr{P} i c^{\tau}(G / S)$ to $\mathrm{B} \mathbb{G}_{\mathrm{m}}$ that maps a line bundle $\mathscr{L}$ to $y^{*} \mathscr{L}$. Using 7.8, we see that the morphism $f_{0}(x) \cdot a_{T}(y)$ is 2-isomorphic to the morphism that maps a line bundle $\mathscr{L}$ to $\left(x^{*} \mathscr{L}\right) \otimes\left(e^{*} \mathscr{L}\right)^{-1} \otimes\left(y^{*} \mathscr{L}\right)$. Hence, to get the expected $\alpha$, we need a functorial isomorphism between $(x+y)^{*} \mathscr{L}$ and $\left(x^{*} \mathscr{L}\right) \otimes\left(e^{*} \mathscr{L}\right)^{-1} \otimes\left(y^{*} \mathscr{L}\right)$, for any line bundle $\mathscr{L}$ in $\mathscr{P}_{i c^{\tau}}(G / S)$. This is provided by corollary 6.4 (2). Since the local 2-isomorphisms $\alpha$ are canonical, they glue together and yield a global $\alpha$ over $S$.

For further use, we record here a lemma that ensures the compatibility of different isomorphisms introduced so far.

Lemma 7.9. Let $X$ be an algebraic stack over a base scheme $S$. Assume that $f: X \rightarrow S$ locally has sections in the fppf topology and that $\mathscr{O}_{S} \rightarrow f_{*} \mathscr{O}_{X}$ is universally an isomorphism. 
(a) Assume that $P:=\operatorname{Pic}_{X / S}^{\tau}$ is a duabelian group. Then the composite morphism

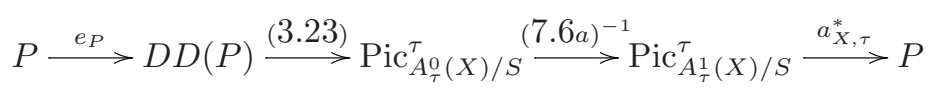

is the identity of $P$. In particular $a_{X, \tau}^{*}$ is an isomorphism.

(b) Assume that $P:=\mathrm{Pic}_{X / S}$ is Cartier (hence $A^{0}(X)=B P^{D}$ ). Then the morphism

$$
P \stackrel{e_{P}}{\longrightarrow} D D(P) \stackrel{(3.26)}{\longrightarrow} \operatorname{Pic}_{\left.A^{0}(X) / S \stackrel{(7.6)}{\longrightarrow}\right)^{-1}}^{\longrightarrow} \operatorname{Pic}_{A^{1}(X) / S} \stackrel{a_{X}^{*}}{\longrightarrow} P
$$

is the identity of $P$. In particular $a_{X}^{*}$ is an isomorphism.

Proof. Let us prove (b). It suffices to prove the statement for $S$-points of $P$ (base change). Let $\lambda \in P(S)$. Since the statement is fppf local, we can assume that $X$ has an $S$-point $x_{0}$ and that $\lambda$ is induced by an invertible sheaf $\mathscr{L}$ on $X$. Then $a_{X}^{*} \circ(7.6 b)^{-1}$ is induced by the pullback of invertible sheaves along the morphism $\varphi_{x_{0}^{*}}^{-1} \circ a_{X}: X \rightarrow A^{0}(X) \subset D(\mathscr{P} i c(X / S))$ that maps a point $x \in X(U)$ to $x^{*}-x_{0}^{*}$. Hence $a_{X}^{*} \circ(\overline{7.6} b)^{-1}\left(\omega\left(e_{P}(\lambda)\right)\right)$ is the class in $P(S)$ of the invertible sheaf corresponding to the morphism

$$
X \stackrel{\varphi_{x_{0}^{*}}^{-1} \circ a_{X}}{\longrightarrow} A^{0}(X) \stackrel{e_{P}(\lambda)}{\longrightarrow} \mathrm{B} \mathbb{G}_{\mathrm{m}} .
$$

The latter morphism maps a point $x \in X(U)$ to $e_{P}(\lambda)\left(x^{*}-x_{0}^{*}\right)=\left(x^{*}-x_{0}^{*}\right)(\lambda)=\left(x^{*} \mathscr{L}\right) \otimes\left(x_{0}^{*} \mathscr{L}\right)^{-1}$. Hence it corresponds to the invertible sheaf $\mathscr{L} \otimes\left(f^{*} x_{0}^{*} \mathscr{L}\right)^{-1}$ on $X$, whose class in $P(S)$ is equal to $\lambda$. The last assertion is obvious since $e_{P}$ and (3.26) are isomorphisms. The proof of (a) is very similar and left to the reader. Note by the way that both (a) and (b) are related to the following fact. Let us denote by $a_{X}$ the canonical morphism $X \rightarrow D(\mathscr{P})$ where $\mathscr{P}=\mathscr{P} i c(X / S)$. Then the composition

$$
\mathscr{P} \stackrel{e_{P}}{\longrightarrow} D D(\mathscr{P}) \stackrel{\omega}{\longrightarrow} \mathscr{P} i c(D(\mathscr{P})) \stackrel{a_{X}^{*}}{\longrightarrow} \mathscr{P}
$$

is the identity of $\mathscr{P}$ (without any assumption on $f$ ).

\section{UNIVERSAL PROPERTIES}

The following theorem generalizes FGA VI, théorème 3.3 (iii) [33, exp. 236]. Note that an explicit comparison is made in Corollary 8.4 .

Theorem 8.1. Let $X$ be an algebraic stack over a base scheme S. Assume that the structural morphism $f: X \rightarrow S$ locally has sections in the fppf topology, that $\mathscr{O}_{S} \rightarrow f_{*} \mathscr{O}_{X}$ is universally an isomorphism, and that the Picard functor $\mathrm{Pic}_{X / S}^{\tau}$ is a duabelian group (see 2.17). Then the Albanese morphism

$$
a_{X}: X \longrightarrow A_{\tau}^{1}(X)
$$

is initial among maps to torsors under abelian stacks (2.15), in the following sense. For any triple $(B, T, b)$ where $B$ is an abelian stack, $T$ is a $B$-torsor and $b: X \rightarrow T$ is a morphism of algebraic stacks, there is a triple $\left(c_{0}, c_{1}, \gamma\right)$ where $c_{0}: A_{\tau}^{0}(X) \rightarrow B$ is a homomorphism of commutative group stacks, $c_{1}: A_{\tau}^{1}(X) \rightarrow T$ is a $c_{0}$-equivariant morphism, and $\gamma$ is a 2-isomorphism $c_{1} \circ a_{X} \Rightarrow b$. Such a triple $\left(c_{0}, c_{1}, \gamma\right)$ is unique up to a unique isomorphism.

The proof of this theorem occupies most of this section. Actually we will prove a slightly more precise statement (Theorem 8.3 below). Keeping the assumptions of 8.1. let us first define the 2-category $\mathscr{T}$ of maps from $X$ to torsors under abelian stacks. An object of $\mathscr{T}$ is a triple $(B, T, b)$ as in 8.1. A morphism from $(B, T, b)$ to another object $\left(B^{\prime}, T^{\prime}, b^{\prime}\right)$ is a triple $\left(c_{0}, c_{1}, \gamma\right)$ where $c_{0}: B \rightarrow B^{\prime}$ is a homomorphism of abelian stacks, $c_{1}: T \rightarrow T^{\prime}$ is a $c_{0}$-equivariant morphism, and $\gamma$ is a 2-isomorphism $c_{1} \circ b \Rightarrow b^{\prime}$. A 2-morphism from $\left(c_{0}, c_{1}, \gamma\right)$ to $\left(d_{0}, d_{1}, \delta\right)$ is a pair $\left(\varepsilon_{0}, \varepsilon_{1}\right)$ where $\varepsilon_{0}: c_{0} \Rightarrow d_{0}$ is a 2-isomorphism of additive morphisms, and $\varepsilon_{1}: c_{1} \Rightarrow d_{1}$ is a 2-isomorphism of equivariant morphisms, i.e. we require that $\varepsilon_{1}$ is compatible to the 2 -isomorphisms that make $c_{1}$ and $d_{1}$ equivariant. We also require $\varepsilon_{1}$ to be compatible with $\gamma$ and $\delta$, i.e. for any object $x$ of $X$, $\varepsilon_{1}^{b(x)}=\delta_{x}^{-1} \circ \gamma_{x}$. 
Lemma 8.2. The automorphism groups of 1-morphisms in $\mathscr{T}$ are trivial. Hence, we may regard the 2-category $\mathscr{T}$ as an ordinary category (that we still denote by $\mathscr{T}$ ).

Proof. Let $(B, T, b)$ and $\left(B^{\prime}, T^{\prime}, b^{\prime}\right)$ be two objects of $\mathscr{T}$ and let $\left(c_{0}, c_{1}, \gamma\right)$ be a morphism from the one to the other. We have to prove that any automorphism $\left(\varepsilon_{0}, \varepsilon_{1}\right)$ of $\left(c_{0}, c_{1}, \gamma\right)$ is trivial. Since $D(B)$ and $D\left(B^{\prime}\right)$ are sheaves (3.23), any automorphism of $D\left(c_{0}\right)$ is trivial and since $D($. is a 2-antiequivalence, $\varepsilon_{0}=\mathrm{id}_{c_{0}}$. Now let us prove that for an object $t$ of $T$, $\varepsilon_{1}^{t}$ is the identity of $c_{1}(t)$. The question is local on $S$ so we may assume that $X(S)$ is nonempty. Let $x \in X(S)$. Since $T$ is a $B$-torsor, there exist an object $g$ of $B$ and an isomorphism $\varphi: g \cdot b(x) \rightarrow t$. Now using the various compatibility conditions on $\varepsilon_{1}$ and its functoriality, we check successively that $\varepsilon_{1}^{b(x)}, \varepsilon_{1}^{g . b(x)}$ and $\varepsilon_{1}^{t}$ are trivial.

Now let $\mathscr{G}$ be the category of pairs $(A, a)$ where $A$ is a duabelian group and $a: A \rightarrow \operatorname{Pic}_{X / S}^{\tau}$ is a morphism of group algebraic spaces. A morphism in $\mathscr{G}$ from $(A, a)$ to $\left(A^{\prime}, a^{\prime}\right)$ is a homomorphism $d: A \rightarrow A^{\prime}$ of group schemes such that $a^{\prime} d=a$. There is a natural functor $\Phi: \mathscr{T} \rightarrow \mathscr{G}$ defined as follows. For an object $(B, T, b)$ of $\mathscr{T}, \Phi(B, T, b)$ is the pair $(A, a)$ where $A=D(B)$ and the homomorphism $a$ is the composition:

$$
a: D(B) \stackrel{3.23}{\longrightarrow} \mathrm{Pic}_{B / S}^{\tau} \stackrel{\sqrt{7.6})^{-1}}{\longrightarrow} \mathrm{Pic}_{T / S}^{\tau} \stackrel{b^{*}}{\longrightarrow} \mathrm{Pic}_{X / S}^{\tau}
$$

The functor $\Phi$ maps a morphism $\left(c_{0}, c_{1}, \gamma\right)$ in $\mathscr{T}$ to $D\left(c_{0}\right)$. This is indeed a morphism in $\mathscr{G}$ using the functoriality of the isomorphisms 3.23 and 7.6 .

Theorem 8.1 exactly means that the object $\left(A_{\tau}^{0}(X), A_{\tau}^{1}(X), a_{X}\right)$ is initial in the category $\mathscr{T}$. Using 7.9 (a), we see that $\Phi$ maps this object to $\left(D D\left(\operatorname{Pic}_{X / S}^{\tau}\right), e_{\operatorname{Pic}_{X / S}^{\tau}}^{-1}\right)$. Since the latter is final in $\mathscr{G}$, Theorem 8.1 is a consequence of 8.3 below.

Theorem 8.3. The functor $\Phi: \mathscr{T} \rightarrow \mathscr{G}$ is an anti-equivalence of categories.

Proof. Let us first prove that $\Phi$ is essentially surjective. Let $(A, a)$ be an object of $\mathscr{G}$. Let $B:=D(A)$. The dual of $a$ is a homomorphism from $D\left(\operatorname{Pic}_{X / S}^{\tau}\right)$ to $B$. Let $T$ be the $B$-torsor $A_{\tau}^{1}(X) \wedge^{A_{\tau}^{0}(X), D(a)} B$ obtained from $A_{\tau}^{1}(X)$ by extension of scalars along $D(a)$ (see 5.6). There is a natural $D(a)$-equivariant morphism $c_{1}: A_{\tau}^{1}(X) \rightarrow T$. Let $b=c_{1} \circ a_{X}$. The triple $(B, T, b)$ is an object of $\mathscr{T}$. Let us denote by $(D D(A), \alpha)$ its image by $\Phi$. Using various functorialities 3.23 . 7.6 and 4.2) and 7.9, we check that the diagram

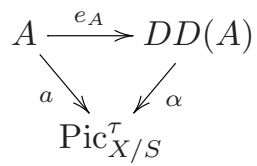

commutes. Hence $e_{A}$ is an isomorphism in $\mathscr{G}$ from $(A, a)$ to $\Phi(B, T, b)$.

Now let us prove that $\Phi$ is fully faithful. Let $\beta=(B, T, b)$ and $\beta^{\prime}=\left(B^{\prime}, T^{\prime}, b^{\prime}\right)$ be two objects of $\mathscr{T}$. For any $S$-scheme $U$, let $X_{U}$ denote $X \times{ }_{S} U$ and let $\mathscr{T}_{U}$ (resp. $\mathscr{G}_{U}$ ) denote the analog of $\mathscr{T}$ (resp. $\mathscr{G}$ ) over the base scheme $U$. In other words $\mathscr{T}_{U}$ is the category of maps from $X_{U}$ to torsors under abelian stacks (over $U$ ), and similarly for $\mathscr{G}_{U}$. Let $H_{\mathscr{T}}$ and $H_{\mathscr{G}}$ be the presheaves defined by $H_{\mathscr{T}}(U)=\operatorname{Hom}_{\mathscr{T}_{U}}\left(\beta_{U}, \beta_{U}^{\prime}\right)$ and $H_{\mathscr{G}}(U)=\operatorname{Hom}_{\mathscr{G}_{U}}\left(\Phi\left(\beta_{U}^{\prime}\right), \Phi\left(\beta_{U}\right)\right)$. The functor $\Phi$ extends to a morphism of presheaves $H_{\Phi}: H_{\mathscr{T}} \rightarrow H_{\mathscr{G}}$ and saying that $\Phi$ is fully faithful precisely means that $H_{\Phi}(S)$ is bijective. We will prove that $H_{\Phi}$ is an isomorphism. Since $U \mapsto \operatorname{Hom}\left(D\left(B^{\prime}\right)_{U}, D(B)_{U}\right)$ is a sheaf (resp. $U \mapsto \operatorname{Hom}\left(B_{U}, B_{U}^{\prime}\right)$ is a stack), the presheaf $H_{\mathscr{G}}$ (resp. $H_{\mathscr{T}}$ ) is actually an fppf sheaf, so the question is local on $S$ in the fppf topology. We can then assume that $X(S)$ is nonempty and we are back to prove that $\Phi$ is fully faithful. Let us fix an object $x_{0} \in X(S)$. We can now asssume that $T=B$ and that the image $e$ of $x_{0}$ by $b$ is a neutral element of $B$.

For the faithfulness, let $\left(c_{0}, c_{1}, \gamma\right)$ and $\left(d_{0}, d_{1}, \delta\right)$ be two morphisms from $\beta$ to $\beta^{\prime}$ such that $D\left(c_{0}\right)=D\left(d_{0}\right)$. We want to find a 2-isomorphism $\left(\varepsilon_{0}, \varepsilon_{1}\right)$ from $\left(c_{0}, c_{1}, \gamma\right)$ to $\left(d_{0}, d_{1}, \delta\right)$. Since $D($. is a 2-antiequivalence, we already know that there is a unique 2-isomorphism of additive morphisms $\varepsilon_{0}: c_{0} \Rightarrow d_{0}$ such that $D\left(\varepsilon_{0}\right)$ is the identity of $D\left(c_{0}\right)$. It remains to construct $\varepsilon_{1}: c_{1} \Rightarrow d_{1}$. Let $g$ 
be an object of $B$. We choose an isomorphism $\varphi: g . e \rightarrow g$ and we define $\varepsilon_{1}^{g}$ by the commutative diagram:

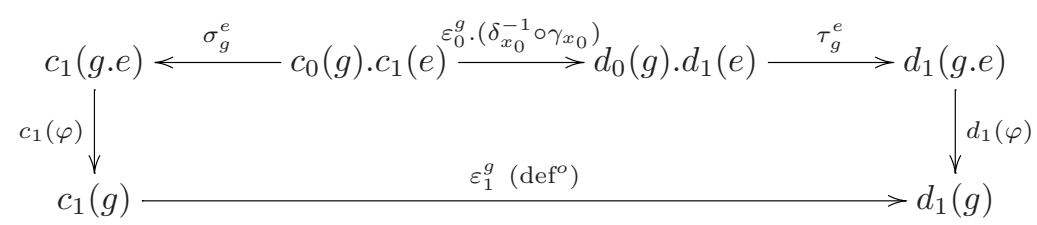

where $\sigma$ and $\tau$ are the 2-isomorphisms that make $c_{1}$ and $d_{1}$ equivariant (see 5.1 (ii)). We claim that $\varepsilon_{1}^{g}$ does not depend on the choice of $\varphi$. To see this, it suffices to prove that for any automorphism $\psi$ of g.e the following diagram of solid arrows commutes:

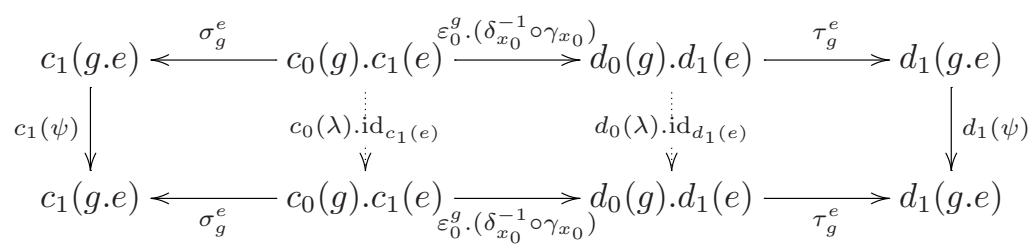

There is a unique automorphism $\lambda$ of $g$ such that $\lambda \cdot \operatorname{id}_{e}=\psi$. Then the commutativity of the above diagram follows from the functoriality of $\sigma, \varepsilon_{0}$ and $\tau$. Since $\varepsilon_{1}^{g}$ does not depend on the choice of $\varphi$, we see that the collection of the $\varepsilon_{1}^{g}$ for all objects $g$ of $B$ is functorial, i.e. it defines a 2-isomorphism $\varepsilon_{1}: c_{1} \Rightarrow d_{1}$. Moreover, for all objects $t$ and $g$ of $B$, the following diagram commutes:

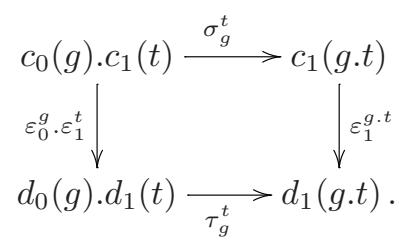

(Choose an isomorphism $\varphi: t . e \rightarrow t$ to define $\varepsilon_{1}^{t}$ with the diagram $\left(^{*}\right)$ above. Then choose the isomorphism $(g . t) . e \stackrel{\text { can. }}{\rightarrow}$ g. (t.e) $\stackrel{\mathrm{id}_{g} \cdot \varphi}{\rightarrow}$ g.t to define $\varepsilon_{1}^{g . t}$. To see that the resulting diagram is commutative, use among other facts the functoriality of $\sigma, \tau, \varepsilon_{0}$ and the hexagon condition 5.1 (ii).) This means that $\varepsilon_{1}$ is a 2-isomorphism of equivariant morphisms. It remains to prove that for any object $x$ of $X, \varepsilon_{1}^{b(x)}=\delta_{x}^{-1} \circ \gamma_{x}$. Using the above diagram with $g=t=e$, we see that $\varepsilon_{0}^{e} .\left(\delta_{x_{0}}^{-1} \circ \gamma_{x_{0}}\right)=\varepsilon_{0}^{e} . \varepsilon_{1}^{e}$ from which we deduce that $\varepsilon_{1}^{b\left(x_{0}\right)}=\delta_{x_{0}}^{-1} \circ \gamma_{x_{0}}$. Now, the map $x \mapsto \delta_{x} \circ \varepsilon_{1}^{b(x)} \circ \gamma_{x}^{-1}$ is functorial and defines a morphism of algebraic stacks from $X$ to $H^{-1}\left(B^{\prime}\right)$. By Lemma 3.21] this morphism must be constant and this proves the desired equality for all $x$. This finishes the proof of the fact that $\Phi$ is faithful.

To prove that $\Phi$ is full, we keep the same notations for the objects $\beta$ and $\beta^{\prime}$. Let $(D(B), a)$ and $\left(D\left(B^{\prime}\right), a^{\prime}\right)$ be their images in $\mathscr{G}$, and let $d: D\left(B^{\prime}\right) \rightarrow D(B)$ be a morphism in $\mathscr{G}$. In particular $a d=a^{\prime}$. Since $D($.$) is an antiequivalence, we know that there is a homomorphism c_{0}: B \rightarrow B^{\prime}$ such that $D\left(c_{0}\right)=d$. We take $c_{1}=c_{0}: B \rightarrow B^{\prime}$ which is clearly $c_{0}$-equivariant, and to conclude the proof it suffices to check that the morphisms $b^{\prime}$ and $c_{0} \circ b$ from $X$ to $B^{\prime}$ are isomorphic. By 7.3 there is a commutative diagram

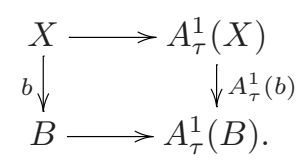

With the notations of 7.7 the bottom horizontal arrow is an $f_{0}$-equivariant isomorphism of torsors. Hence we deduce a morphism of torsors $A_{\tau}^{1}(X) \rightarrow B$ which is equivariant under the homomorphism

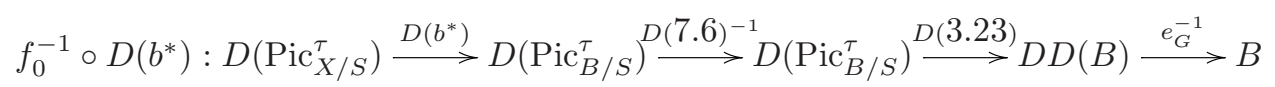


and through which $b$ factorizes (here, since $T=B$, the isomorphism (7.6) is actually the identity). Choosing a trivialization of the torsor $A_{\tau}^{1}(X)$, we find a morphism $u: X \rightarrow D\left(\operatorname{Pic}_{X / S}^{\tau}\right)$ such that $b$ factorizes as $f_{0}^{-1} \circ D\left(b^{*}\right) \circ u$. Similarly $b^{\prime}$ factorizes through the same $u$ as $f_{0}^{\prime-1} \circ D\left(b^{\prime *}\right) \circ u$. It now suffices to prove that the homomorphisms of commutative group stacks $c_{0} f_{0}^{-1} D\left(b^{*}\right)$ and $f_{0}^{\prime-1} D\left(b^{\prime *}\right)$ are isomorphic. Applying $D($.$) , this is equivalent to$

$$
D\left(f_{0}^{-1} D\left(b^{*}\right)\right) \circ d=D\left(f_{0}^{\prime-1} \circ D\left(b^{*}\right)\right) .
$$

Using 4.2, we see that $D\left(f_{0}^{-1} D\left(b^{*}\right)\right)$ is equal to $e_{\mathrm{Pic}_{X / S}^{\tau}} \circ a$ and $D\left(f_{0}^{\prime-1} D\left(b^{*}\right)\right)$ to $e_{\mathrm{Pic}_{X / S}^{\tau}} \circ a^{\prime}$. Hence the desired equality follows from the assumption $a d=a^{\prime}$.

Corollary 8.4. Under the assumptions of 8.1, assume that $\mathrm{Pic}_{X / S}$ has an abelian subscheme $A$, the underlying subset of which is $\mathrm{Pic}_{X / S}^{0}$. Let us denote by $\operatorname{Alb}^{1}(X)$ the torsor obtained from $A^{1}(X)$ by extension of scalars along $D\left(\mathrm{Pic}_{X / S}\right) \rightarrow D(A)$, and by $u$ the composed morphism:

$$
u: X \stackrel{a}{\longrightarrow} A^{1}(X) \longrightarrow \operatorname{Alb}^{1}(X) .
$$

Then:

(i) $u$ is initial among morphisms from $X$ to torsors under abelian schemes. In particular this proves that $u$ coincides with the classical Albanese morphism of FGA VI, théorème 3.3 (iii) [33. exp. 236].

(ii) Via the canonical morphism $A_{\tau}^{1}(X) \rightarrow \operatorname{Alb}^{1}(X)$, the classical Albanese torsor $\operatorname{Alb}^{1}(X)$ is the coarse moduli space of the Albanese stack $A_{\tau}^{1}(X)$. If $\mathrm{Pic}_{X / S} / \mathrm{Pic}_{X / S}^{\tau}$ is a twisted lattice, then $\operatorname{Alb}^{1}(X)$ is also the coarse moduli space of $A^{1}(X)$.

Proof. Let $Q$ denote the quotient sheaf $\mathrm{Pic}_{X / S}^{\tau} / A$. By Artin's representability theorem [39, 10.4] it is an algebraic space. It is proper and flat because so is $\operatorname{Pic}_{X / S}^{\tau}$. We first prove that any morphism $B \rightarrow \mathrm{Pic}_{X / S}^{\tau}$ where $B$ is an abelian scheme factorizes through $A$. If $S$ is the spectrum of a field then $A$ is equal to $\left(\mathrm{Pic}_{X / S}^{0}\right)_{\text {réd }}$ and the claim follows from the universal property of the reduced subscheme. In the general case it suffices to prove that the composition $\lambda: B \rightarrow Q$ is zero. But this morphism is constant on the fibers (by the case where $S$ is a field) hence it is constant by [42, 6.1]. Since it maps 0 to 0 it is then the trivial morphism.

Let us now prove that $Q$ is finite. It only remains to prove that it is quasi-finite, so for this question we may assume that $S$ is the spectrum of a field. By assumption there is an exact sequence $0 \rightarrow B \rightarrow \mathrm{Pic}_{X / S}^{\tau} \rightarrow F \rightarrow 0$ where $F$ is a finite flat commutative group scheme and $B$ is an abelian scheme. By the above $B \rightarrow \operatorname{Pic}_{X / S}^{\tau}$ factorizes through $A$, and we get a morphism $F \rightarrow Q$. This morphism is proper, smooth and surjective because so are the morphisms $\mathrm{Pic}_{X / S}^{\tau} \rightarrow F$ and $\mathrm{Pic}_{X / S}^{\tau} \rightarrow Q$, whence the assertion.

Let us prove (i). Let $b: X \rightarrow T$ be a morphism from $X$ to a $B$-torsor, where $B$ is an abelian scheme. By 8.1. there exists a homomorphism $c_{0}: A_{\tau}^{0}(X) \rightarrow B$ and a $c_{0}$-equivariant morphism of torsors $c_{1}: A_{\tau}^{1}(X) \rightarrow T$ such that $c_{1} \circ a_{X, \tau}=b$. The dual $D\left(c_{0}\right): B^{t} \rightarrow \mathrm{Pic}_{X / S}^{\tau}$ factorizes (uniquely) through $A$, by the above. Dualizing, we get a morphism $\overline{c_{0}}: D(A) \rightarrow B$ through which $c_{0}$ factorizes. Then there is a unique $\overline{c_{0}}$-equivariant morphism $\overline{c_{1}}: \operatorname{Alb}^{1}(X) \rightarrow B$ such that $\overline{c_{1}} u=b$ and this concludes the proof.

Now let us prove (ii). The assertion is equivalent to saying that $A_{\tau}^{1}(X) \rightarrow \operatorname{Alb}^{1}(X)$ is an $f p p f$-gerbe. This question is fppf-local on $S$ so we may assume that $X$ has an $S$-point. Then the torsors are trivial and we have to prove that $A_{\tau}^{0}(X)$ is a gerbe over $A^{t}$. But, since $Q$ is finite and flat, the exact sequence $0 \rightarrow A \rightarrow \operatorname{Pic}_{X / S}^{\tau} \rightarrow Q \rightarrow 0$ induces by 3.18 an exact sequence $0 \rightarrow B Q^{D} \rightarrow A_{\tau}^{0}(X) \rightarrow A^{t} \rightarrow 0$. If $M:=\mathrm{Pic}_{X / S} / \mathrm{Pic}_{X / S}^{\tau}$ is a twisted lattice, then we see similarly that $A^{0}(X)$ is an $M^{D}$-gerbe over $A_{\tau}^{0}(X)$ and the last assertion follows.

Example 8.5. Let $k$ be a field and let $C$ be a geometrically integral smooth projective curve over $k$. Then $\mathrm{Pic}_{C / k}^{\tau}=\mathrm{Pic}_{C / k}^{0}$ and it is an abelian variety. Hence by 8.4 the Albanese stack $A_{\tau}^{1}(X)$ is isomorphic to the classical Albanese torsor $\operatorname{Alb}^{1}(C / k)$.

Example 8.6. Let $f: X \rightarrow S$ be a proper and flat morphism of schemes, with $f_{*} \mathscr{O}_{X}$ universally isomorphic to $\mathscr{O}_{S}$ and $S$ noetherian. Let $\mathscr{L}$ be an invertible sheaf on $X$ and $r$ a positive integer. Let 
us denote by $\mathscr{X}=\sqrt[r]{\mathscr{L}}$ the stack that classifies $r$-th roots of $\mathscr{L}$ (see [19, 2.2.6] or [17, 5.3] for the precise definition). Let us describe the Albanese morphism of $\mathscr{X}$. The stack $\mathscr{X}$ is a $\mu_{r}$-gerbe over $X$. By [17, 5.3], there is an exact sequence of sheaves $0 \rightarrow \operatorname{Pic}_{X / S} \rightarrow \operatorname{Pic}_{\mathscr{X} / S} \rightarrow \mathbb{Z} / r \mathbb{Z} \rightarrow 0$. By 3.18 the dual sequence

$$
0 \longrightarrow B \mu_{r} \longrightarrow A^{0}(\mathscr{X}) \longrightarrow A^{0}(X) \longrightarrow 0
$$

is exact hence $A^{0}(\mathscr{X})$ is a $\mu_{r}$-gerbe over $A^{0}(X)$. Let us now assume that $\mathrm{Pic}_{X / S}^{\tau}$ is an abelian scheme over $S$ (hence equal to $\operatorname{Pic}_{X / S}^{0}$ ). Let $Q$ be the image $f p p f$ sheaf of $\operatorname{Pic}_{\mathscr{X} / S}^{\tau}$ in $\mathbb{Z} / r \mathbb{Z}$. Assume that $Q$ is an open and closed group subscheme of $\mathbb{Z} / r \mathbb{Z}$. By [18, 3.3.2] there is an exact sequence $0 \rightarrow \operatorname{Pic}_{X / S}^{\tau} \rightarrow \operatorname{Pic}_{\mathscr{X} / S}^{\tau} \rightarrow Q \rightarrow 0$. Then by 3.18, the dual sequence

$$
0 \rightarrow D(Q) \rightarrow A_{\tau}^{0}(\mathscr{X}) \rightarrow A_{\tau}^{0}(X) \rightarrow 0
$$

is exact hence $A_{\tau}^{0}(\mathscr{X})$ is a $Q^{D}$-gerbe over $A_{\tau}^{0}(X)$. To describe the morphism $\mathscr{X} \rightarrow A_{\tau}^{1}(\mathscr{X})$, let us assume that $\mathscr{X}$ has an $S$-point $x_{0}$ (this is true fppf-locally on $S$ ). Then using the point $a_{\mathscr{X}}\left(x_{0}\right)=x_{0}^{*}$, the torsor trivializes and we will describe the resulting morphism $\mathscr{X} \rightarrow A_{\tau}^{0}(\mathscr{X})$. There is a commutative diagram

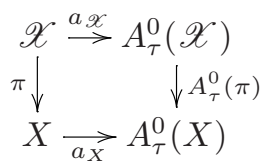

in which, by 8.4, the bottom map $a_{X}$ is the classical Albanese morphism, mapping $\pi\left(x_{0}\right)$ to 0 . Let $\mathscr{X}_{0}$ denote the fiber $\pi^{-1}\left(\pi\left(x_{0}\right)\right)$ and $\mathscr{A}_{0} \simeq D(Q)$ the kernel of $A_{\tau}^{0}(\pi)$, and let us compute the induced morphism $\mathscr{X}_{0} \rightarrow \mathscr{A}_{0}$. The commutative diagram

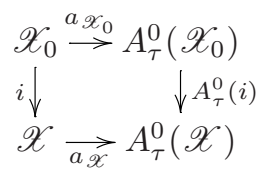

induces a factorization of the morphism $\mathscr{X}_{0} \rightarrow \mathscr{A}_{0}$ through $a_{\mathscr{X}_{0}}$. But the stack $\mathscr{X}_{0}$ is a trivial $\mu_{r}$-gerbe over $S$. Hence $a_{\mathscr{X}_{0}}$ is an isomorphism (see 7.4 and 7.8). In the end, through the abovementioned identifications, the morphism $\mathscr{X}_{0} \rightarrow \mathscr{A}_{0}$ is the dual of the inclusion of $Q$ into $\mathbb{Z} / r \mathbb{Z}$.

To conclude this example, let us discuss a little bit this assumption that $Q$ is an open and closed group subscheme of $\mathbb{Z} / r \mathbb{Z}$. If $[\mathscr{L}] \in \operatorname{Pic}_{X / S}^{\tau}$ or if $\mathscr{L}$ has an $r$-th root on $X$, then $Q=\mathbb{Z} / r \mathbb{Z}$ so the condition holds. If $X$ is smooth and projective over $S$, then $Q$ is automatically a closed subscheme of $\mathbb{Z} / r \mathbb{Z}$. However, even with that latter assumption, it seems that $Q$ does not need to be open in $\mathbb{Z} / r \mathbb{Z}$. Its openness is equivalent to the flatness of $\operatorname{Pic}_{\mathscr{X} / S}^{\tau}$ over $S$. (Indeed, since the morphism $Q \rightarrow \mathbb{Z} / r \mathbb{Z}$ is a monomorphism of finite type, it is an open immersion if and only if it is flat, i.e. if and only if $Q$ is flat over $S$. Then the claim follows from the fact that flatness is local on the source, and that $\operatorname{Pic}_{\mathscr{X} / S}^{\tau} \rightarrow Q$ is an fppf epimorphism.) In particular $Q$ is open if $\mathrm{Pic}_{X / S}$ is flat over $S$.

Example 8.7. Very similarly, let us consider the case of a smooth twisted curve $\mathscr{C} \rightarrow C$ as defined by Abramovich and Vistoli [6]. By Cadman [19, 2.2.4 and 4.1], $\mathscr{C}$ can be described as a root stack $\sqrt[r_{1}]{\left(\mathscr{L}_{1}, s_{1}\right)} \times_{S} \cdots \times_{S} \sqrt[r_{n}]{\left(\mathscr{L}_{n}, s_{n}\right)}$, where $\mathscr{L}_{i}$ is an invertible sheaf on $C$ and $s_{i}$ is a global section of $\mathscr{L}_{i}$. Then by [17, 5.4] $\mathrm{Pic} \mathscr{C}_{\mathscr{C} / S}$ is an extension of $\mathbb{Z} / r_{1} \mathbb{Z} \times_{S} \cdots \times_{S} \mathbb{Z} / r_{n} \mathbb{Z}$ by $\mathrm{Pic}_{C / S}$. Considering the dual sequence, which is exact by 3.18 , we see as above that $A^{0}(\mathscr{C})$ is a gerbe over $A^{0}(C)$, banded by $\mu_{r_{1}} \times \cdots \times \mu_{r_{n}}$. Let $Q$ denote the image of $\operatorname{Pic}_{\mathscr{C} / S}^{\tau}$ in $\prod \mathbb{Z} / r_{i} \mathbb{Z}$. It is an open and closed group subscheme (because $C$ is a smooth and projective family of curves). The group $\mathrm{Pic}_{C / S}^{\tau}$ is an abelian scheme, hence the exact sequence $0 \rightarrow \operatorname{Pic}_{C / S}^{\tau} \rightarrow \operatorname{Pic}_{\mathscr{C} / S}^{\tau} \rightarrow Q \rightarrow 0$ shows that $A_{\tau}^{0}(\mathscr{C})$ is an abelian stack (it is a $Q^{D}$-gerbe over the abelian scheme $\left.\left(\mathrm{Pic}_{C / S}^{0}\right)^{t}\right)$.

Theorem 8.8. Let $X$ be an algebraic stack over a base scheme $S$. Assume that the structural morphism $f: X \rightarrow S$ locally has sections in the fppf topology, that $\mathscr{O}_{S} \rightarrow f_{*} \mathscr{O}_{X}$ is universally an isomorphism, and that the Picard functor $\mathrm{Pic}_{X / S}$ is a Cartier group scheme (see 3.7). Then the Albanese morphism of $X$

$$
a_{X}: X \longrightarrow A^{1}(X)
$$


is initial among maps to torsors under classifying stacks of Cartier group schemes (that is, maps to gerbes banded by Cartier group schemes).

Proof. The proof of 8.1 holds verbatim, mutatis mutandis. More precisely, replace everywhere Pic ${ }^{\tau}$ with Pic, duabelian with Cartier, $A_{\tau}^{i}$ with $A^{i}$, "abelian stack" with "classiying stack of a Cartier group scheme", 3.23 with 3.26, 7.6 (a) with 7.6 (b), and 7.9 (a) with 7.9 (b). You also need a variant of Lemma 3.21 where the affine target $Y$ is replaced with a Cartier group scheme. To prove it, we proceed as follows. If $Y$ is a finite flat group scheme, or of multiplicative type, then 3.21 itself applies. If $Y$ is a twisted lattice then any $S$-morphism $X \rightarrow Y$ must be constant because the geometric fibers of $X$ are connected. For an arbitrary Cartier group scheme, the result follows by induction on the number of extensions. Notice in particular that the analogs in this context of 8.2 and 8.3 hold.

\section{Application to Rational Varieties}

The "universal torsors" and the so-called "elementary obstruction" were introduced by ColliotThélène and Sansuc in a series of Notes $([21,22,23])$ and in the foundational article [24]. These are the key ingredients of a general method which proved to be very useful in the study of rational points of some algebraic varieties. One of the main tools is the following fundamental exact sequence. Let $f: X \rightarrow S$ be a morphism of schemes. Throughout this section we assume that $\mathscr{O}_{S} \rightarrow f_{*} \mathscr{O}_{X}$ is universally an isomorphism and that $f$ locally has sections in the fppf topology.

Lemma 9.1 ([22, prop. 1], [24, 2.0]). Let $G$ be an $S$-group scheme of multiplicative type and of finite type. Then there is a functorial exact sequence:

$$
0 \longrightarrow H^{1}(S, G) \stackrel{i_{1}}{\longrightarrow} H^{1}(X, G) \stackrel{\chi}{\longrightarrow} \operatorname{Hom}_{S-g p}\left(G^{D}, \operatorname{Pic}_{X / S}\right) \stackrel{\partial}{\longrightarrow} H^{2}(S, G) \stackrel{i_{2}}{\longrightarrow} H^{2}(X, G)
$$

Remark 9.2. In this sequence, $i_{1}$ and $i_{2}$ are given by pullback along $f$, and the cohomology groups are computed with respect to the fppf topology. Note that if $G$ is smooth they coincide with the étale ones.

Assume moreover that $\operatorname{Pic}_{X / S}$ is representable by a twisted lattice2 (i.e. étale-locally $\mathrm{Pic}_{X / S}$ is the constant sheaf associated with an ordinary free abelian group of finite rank). Its Cartier dual $G_{0}:=\operatorname{Pic}_{X / S}^{D}$ is of multiplicative type and of finite type. According to [22, a universal torsor is by definition a $G_{0}$-torsor $T$ over $X$ (or, by a slight abuse of notation, its class in $H^{1}\left(X, G_{0}\right)$ ) such that $\chi(T)$ is the canonical isomorphism $\lambda_{0}: \operatorname{Pic}_{X / S}^{D D} \rightarrow \operatorname{Pic}_{X / S}$. For each $x \in X(S)$, the unique universal torsor $T_{x} \in H^{1}\left(X, G_{0}\right)$ whose pullback along $x$ is trivial is called the universal torsor associated with $x$. Note that, since $x^{*} T_{x}$ is trivial, the $S$-point $x$ is in the image of $T_{x}(S) \rightarrow X(S)$. The elementary obstruction is the class $\partial\left(\lambda_{0}\right)$ in the group $H^{2}\left(S, G_{0}\right)$. This is an obstruction to the existence of universal torsors. If the elementary obstruction vanishes, then there is a universal torsor, and some natural questions about the $S$-points of $X$ reduce to the same questions on $T$, which in general is arithmetically simpler than $X$.

In this section, we will relate these constructions to the Albanese torsor of section 7 giving by the way a geometric description of the elementary obstruction (in terms of a gerbe). Let $a: X \rightarrow A^{1}(X)$ be the canonical morphism of 7.2. Note that by [3.6, $A^{1}(X)$ is a $G_{0}$-gerbe.

Let $G$ be an $S$-group of multiplicative type and of finite type. We will define maps $\chi^{\prime}$ from $H^{1}(X, G)$ to $\operatorname{Hom}_{S-g p}\left(G^{D}, \operatorname{Pic}_{X / S}\right)$ and $\partial^{\prime}$ from $\operatorname{Hom}_{S-g p}\left(G^{D}, \operatorname{Pic}_{X / S}\right)$ to $H^{2}(S, G)$ and compare them to the $\chi$ and $\partial$ of 9.1 . Let $T$ be a $G$-torsor over $X$. Then $T$ corresponds to a morphism $\varphi_{T}: X \rightarrow B G$. By 8.8 there is up to isomorphism a unique triple $\left(c_{0}, c_{1}, \gamma\right)$ such that $c_{0}:$ $D\left(\mathrm{Pic}_{X / S}\right) \rightarrow B G$ is a morphism of commutative group stacks, $c_{1}: A^{1}(X) \rightarrow B G$ is a $c_{0^{-}}$ equivariant morphism, and $\gamma$ makes the diagram

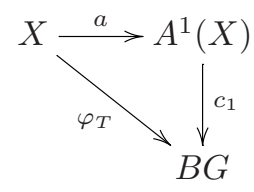

\footnotetext{
${ }^{2}$ This is the case if $S$ is the spectrum of a field $k$, and $X$ is proper and smooth over $k$, and $\bar{k}$-rational.
} 
2-commutative. We define $\chi^{\prime}(T)$ to be the composition of the dual $D\left(c_{0}\right)$ with the canonical isomorphisms as follows:

$$
\chi^{\prime}(T): G^{D} \stackrel{3.5)^{-1}}{\longrightarrow} D(B G) \stackrel{D\left(c_{0}\right)}{\longrightarrow} D D\left(\mathrm{Pic}_{X / S}\right) \stackrel{e_{\mathrm{Pic}_{X / S}}^{-1}}{\longrightarrow} \mathrm{Pic}_{X / S} .
$$

Note that since the commutative group stacks involved here are sheaves, $\chi^{\prime}(T)$ does not depend on the choice of $c_{0}$ into its isomorphism class. Now let $\lambda \in \operatorname{Hom}_{S-g p}\left(G^{D}, \operatorname{Pic}_{X / S}\right)$. We define $\partial^{\prime}(\lambda)$ to be the class in $H^{2}(S, G)$ of the $G$-gerbe (that is, the $B G$-torsor), obtained from the $D\left(\operatorname{Pic}_{X / S}\right)$-torsor $A^{1}(X)$ by extension of scalars (see $5.6 \mathrm{~b}$ )) along the composed morphism

$$
D\left(\operatorname{Pic}_{X / S}\right) \stackrel{D(\lambda)}{\longrightarrow} D\left(G^{D}\right) \stackrel{D \stackrel{D .5}{\longrightarrow}}{\longrightarrow} D D(B G) \stackrel{e_{B G}^{-1}}{\longrightarrow} B G .
$$

\section{Proposition 9.3.}

(i) $\chi^{\prime}$ and $\partial^{\prime}$ are homomorphisms.

(ii) $\chi^{\prime}=\chi$.

(iii) The sequence

$$
0 \longrightarrow H^{1}(S, G) \stackrel{i_{1}}{\longrightarrow} H^{1}(X, G) \stackrel{\chi^{\prime}}{\longrightarrow} \operatorname{Hom}_{S-g p}\left(G^{D}, \operatorname{Pic}_{X / S}\right) \stackrel{\partial^{\prime}}{\longrightarrow} H^{2}(S, G) \stackrel{i_{2}}{\longrightarrow} H^{2}(X, G)
$$

is exact.

(iv) The elementary obstruction $\partial\left(\lambda_{0}\right)$ vanishes if and only if the gerbe $A^{1}(X)$ is trivial (equivalently, if and only if $\left.\partial^{\prime}\left(\lambda_{0}\right)=0\right)$.

Proof. The map $\partial^{\prime}$ is a homomorphism due to 3.2 and 5.7. To prove that $\chi^{\prime}$ is a homomorphism, we can invoke (ii), or proceed directly as follows. Let $T$ and $T^{\prime}$ be two $G$-torsors over $X$ and $\left(c_{0}, c_{1}\right),\left(c_{0}^{\prime}, c_{1}^{\prime}\right)$ the associated pairs of morphisms as above. The product map $c_{1} \cdot c_{1}^{\prime}$ from $A^{1}(X)$ to $B G$ is equivariant under $c_{0} \cdot c_{0}^{\prime}$ and maps the object $a \in A^{1}(X)(X)$ to $T \wedge^{G} T^{\prime}$. Looking at the definition of $\chi^{\prime}$ and using 3.2, we deduce the expected relation $\chi^{\prime}\left(T \wedge^{G} T^{\prime}\right)=\chi^{\prime}(T) \cdot \chi^{\prime}\left(T^{\prime}\right)$.

Let us prove (ii). Let $T$ be a $G$-torsor over $X$. Let us prove that the morphisms $\chi(T)$ and $\chi^{\prime}(T)$ are equal. By [24, 1.5.2 (ii)], the morphism $\chi(T): G^{D} \rightarrow \operatorname{Pic}_{X / S}$ is the morphism that maps a character $\varphi: G \rightarrow \mathbb{G}_{\mathrm{m}}$ to the point of Pic $X / S$ corresponding to the $\mathbb{G}_{\mathrm{m}}$-torsor $T \wedge^{G, \varphi} \mathbb{G}_{\mathrm{m}}$. By construction, the morphism $c_{0}$ is such that $e_{\mathrm{Pic}_{X / S}}^{-1} \circ D\left(c_{0}\right)=\varphi_{T}^{*} \circ(7.6)^{-1} \circ(3.26)$ (see the proof of 8.8). Here (7.6) is the identity, hence $\chi^{\prime}(T)=\varphi_{T}^{*} \circ(3.26) \circ(3.5)^{-1}$ and it suffices to prove that the following diagram commutes:

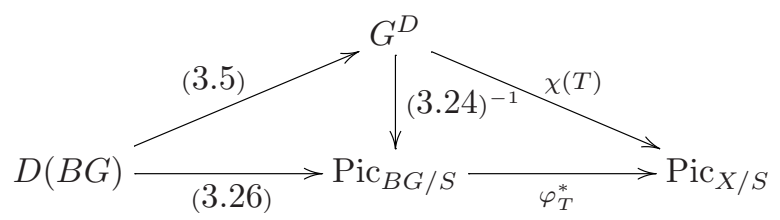

The left triangle commutes because of 3.25 It remains to prove that the right triangle commutes. Since all the constructions commute with base change it suffices to do it on $S$-points. Let $\varphi$ : $G \rightarrow \mathbb{G}_{\mathrm{m}}$ be a character of $G$. By construction of the morphism $(3.24)^{-1}$, it maps $\varphi$ to the class of an invertible sheaf $\mathscr{L}(\varphi)$ such that $\varphi_{T}^{*} \mathscr{L}(\varphi)$ is the class of the $\mathbb{G}_{\mathrm{m}}$-torsor $T \wedge^{G, \varphi} \mathbb{G}_{\mathrm{m}}$ in $\operatorname{Pic}_{X / S}(S)$, as desired.

Now let us prove the exactness of the sequence (iii) in $\operatorname{Hom}_{S-g p}\left(G^{D}, \operatorname{Pic}_{X / S}\right)$. Let $T$ be a $G$ torsor over $X$. The morphism $c_{1}: A^{1}(X) \rightarrow B G$ in the above construction of $\chi^{\prime}(T)$ is equivariant under $c_{0}$. But using 4.2 we see that $c_{0}$ is equal to the morphism $D\left(\operatorname{Pic}_{X / S}\right) \rightarrow B G$ along which we extend the gerbe $A^{1}(X)$ to define $\partial^{\prime}\left(\chi^{\prime}(T)\right)$. This proves that the gerbe we get by extension of scalars is trivial, hence $\partial^{\prime}\left(\chi^{\prime}(T)\right)=0$. Conversely, if $\partial^{\prime}(\lambda)=0$ for some morphism $\lambda: G^{D} \rightarrow \operatorname{Pic}_{X / S}$, this means that the gerbe defining this class is trivial. We have a morphism $A^{1}(X) \rightarrow B G$ that is equivariant under $e_{B G}^{-1} \circ D(3.5) \circ D(\lambda)$. Composing with $a: X \rightarrow A^{1}(X)$, we get a morphism $X \rightarrow B G$ which corresponds to a $G$-torsor $T$ and we check that $\chi^{\prime}(T)=\lambda$. Similarly, the exactness in $H^{2}(S, G)$ is a consequence of the universal property 8.8 
Obviously $\partial\left(\lambda_{0}\right)=0$ if and only if $\partial^{\prime}\left(\lambda_{0}\right)=0$, by (iii). But the map along which we extend the scalars to construct the gerbe defining $\partial^{\prime}\left(\lambda_{0}\right)$ is an isomorphism. Hence $\partial^{\prime}\left(\lambda_{0}\right)=0$ if and only if the gerbe $A^{1}(X)$ is trivial.

Remark 9.4. As one of the referees points out, it is certainly possible, but perhaps painful and not worth the effort, to determine whether $\partial$ and $\partial^{\prime}$ are actually equal or not (they might be opposite). Since both maps are functorial (covariant) in $G$, this is equivalent to $\partial\left(\lambda_{0}\right)=\partial^{\prime}\left(\lambda_{0}\right)$. In other words, $\partial=\partial^{\prime}$ if and only if the elementary obstruction $\partial\left(\lambda_{0}\right)$ is the class in $H^{2}\left(S, G_{0}\right)$ of the gerbe $A^{1}(X)$.

Now let us describe the universal torsors $T_{x}$ in terms of the Albanese morphism $a: X \rightarrow A^{1}(X)$. Let $\eta_{0}: B G_{0} \rightarrow A^{0}(X)$ be the canonical isomorphism of 3.6. If $x \in X(S)$ is an $S$-point of $X$, its image $a(x)$ induces an $\eta_{0}$-equivariant isomorphism of torsors (that is, a trivialization of the $G_{0}$-gerbe $\left.A^{1}(X)\right) \quad B G_{0} \stackrel{\sim}{\rightarrow} A^{1}(X)$ which maps the trivial $G_{0}$-torsor to $a(x)$. Let $T_{x}^{\prime}$ denote the torsor over $X$ corresponding to the composition

$$
X \stackrel{a}{\longrightarrow} A^{1}(X) \stackrel{\sim}{\longleftarrow} B G_{0} .
$$

Proposition 9.5. The torsor $T_{x}^{\prime}$ is (isomorphic to) the universal torsor $T_{x}$ associated with $x$.

Proof. By construction $T_{x}^{\prime}$ is a $G_{0}$-torsor over $X$ whose pullback along $x$ is trivial. Hence is suffices to prove that $\chi\left(T_{x}^{\prime}\right) \in \operatorname{Hom}_{S-g p}\left(G_{0}^{D}, \mathrm{Pic}_{X / S}\right)$ is equal to $\lambda_{0}$. Owing to 9.3 (ii), $\chi\left(T_{x}^{\prime}\right)$ is equal to the composition

$$
\operatorname{Pic}_{X / S}^{D D}=G_{0}^{D} \stackrel{3.5^{-1}}{\longrightarrow} D\left(B G_{0}\right) \stackrel{D\left(\eta_{0}\right)^{-1}}{\longrightarrow} D D\left(\mathrm{Pic}_{X / S}\right) \stackrel{e_{\mathrm{Pic}_{X / S}}^{-1}}{\longrightarrow} \mathrm{Pic}_{X / S}
$$

which is equal to $\lambda_{0}$ due to Lemma 4.6

\section{Applications to Grothendieck's section Conjecture}

Let $X$ be an algebraic stack over a field $k$. Borne and Vistoli define a Nori fundamental gerbe for $X$ as follows (see [10, 5.1]). It is a gerbe $\Pi_{X / k}$ with a $k$-morphism $X \rightarrow \Pi_{X / k}$ that is universal for morphisms to finite stacks (a finite stack is an algebraic stack $\Gamma$ over $k$ with finite diagonal, which admits a flat surjective map $U \rightarrow \Gamma$, where $U$ is a finite scheme over $k$ ). It is unique if it exists [10, 5.2]. By [10, 5.7] an algebraic stack $X$ has a fundamental gerbe if and only if it is inflexible. (See [10, 5.3] for the definition of inflexible stacks. By [10, 5.5], if $X$ is a geometrically connected and geometrically reduced algebraic stack of finite type over $k$, then it is inflexible.) This formalism allows to reformulate Grothendieck's section conjecture as follows: the traditional "section map" is bijective if and only if the natural morphism $X \rightarrow \Pi_{X / k}$ induces a bijection on isomorphism classes of $k$-rational points.

Proposition 10.1. Let $P$ be a Severi-Brauer variety over a field $k$. Let $r$ be its exponent. Then $\operatorname{Pic}(P)$ is generated by an invertible sheaf of degree $r$, which we call $\mathscr{O}_{P}(r)$. Let $f: X \rightarrow P$ be a morphism, where $X$ is an inflexible algebraic stack. Assume that there exists a prime $p$ dividing $r$ and an invertible sheaf $\Lambda$ on $X$, such that $\Lambda^{\otimes p} \simeq f^{*} \mathscr{O}_{P}(r)$. Then $\Pi_{X / k}(k)=\emptyset$.

Proof. Let $Y$ denote the stack of $p$-th roots of $\mathscr{O}_{P}(r)$. For any $k$-scheme $S, Y(S)$ is the category of triples $(x, M, \varphi)$ where $x \in P(S), M$ is an invertible sheaf on $S$ and $\varphi$ is an isomorphism from $M^{\otimes p}$ to $x^{*} \mathscr{O}_{P}(r)$. There is a canonical forgetful morphism $g: Y \rightarrow P$ and there is on $Y$ a canonical $p$-th root $\Omega$ of $g^{*} \mathscr{O}_{P}(r)$, defined by $u^{*} \Omega=M$ if $u: S \rightarrow Y$ is the morphism corresponding to some object $(x, M, \varphi)$ of $Y(S)$. The sheaf $\Lambda$ defines a morphism from $X$ to $Y$, hence a morphism from $\Pi_{X / k}$ to $\Pi_{Y / k}$ and it suffices to prove that $\Pi_{Y / k}$ does not have any $k$-point.

The stack $Y$ is proper, smooth and geometrically integral over $k$. In particular it has an Albanese torsor $A_{\tau}^{1}(Y)$ together with a morphism $a_{Y}: Y \rightarrow A_{\tau}^{1}(Y)$ (see section 7 ), where $A_{\tau}^{1}(Y)$ is a torsor under $A_{\tau}^{0}(Y)=D\left(\operatorname{Pic}_{Y / k}^{\tau}\right)$. The invertible sheaf $\Omega \otimes g^{*} \mathscr{O}_{P}\left(\frac{r}{p}\right)^{-1}$ (which is defined after some extension of $k$ that trivializes $P$ ) defines a section of $\operatorname{Pic}_{Y / k}^{\tau}$ that is mapped to the section $1 \in$ $\mathbb{Z} / p \mathbb{Z}$ under the canonical morphism $\operatorname{Pic}_{Y / k} \rightarrow \mathbb{Z} / p \mathbb{Z}$. This proves that the induced morphism $\operatorname{Pic}_{Y / k}^{\tau} \rightarrow \mathbb{Z} / p \mathbb{Z}$ is surjective. Since $\operatorname{Pic}_{P / k}$ has a trivial torsion component it is also injective and we get an isomorphism $\operatorname{Pic}_{Y / k}^{\tau} \simeq \mathbb{Z} / p \mathbb{Z}$. Hence $A_{\tau}^{0}(Y) \simeq B \mu_{p}$ and $A_{\tau}^{1}(Y)$ is a $\mu_{p}$-gerbe. In 
particular it is a finite gerbe, so by definition of $\Pi_{Y / k}$ there is a morphism $\Pi_{Y / k} \rightarrow A_{\tau}^{1}(Y)$ and to conclude the proof it suffices to prove that $A_{\tau}^{1}(Y)$ has no $k$-points.

Assume that $A_{\tau}^{1}(Y)$ has a $k$-point. Now we claim that any $k$-point of $\operatorname{Pic}_{Y / k}^{\tau}$ is induced by a genuine invertible sheaf on $Y$. This yields a contradiction because then the invertible sheaf $g^{*} \mathscr{O}_{P}\left(\frac{r}{p}\right) \otimes \Omega^{-1}$ is defined over $k$, hence so is $\mathscr{O}_{P}\left(\frac{r}{p}\right)$. To prove the claim, notice that a $k$-point of $A_{\tau}^{1}(Y)$ is a retraction of the natural morphism $\mathrm{B} \mathbb{G}_{\mathrm{m}} \rightarrow \mathscr{P}_{i c^{\tau}}(Y / S)$. Then the natural sequence

$$
0 \longrightarrow \mathrm{B} \mathbb{G}_{\mathrm{m}} \longrightarrow \mathscr{P}_{i c^{\tau}}(Y / k) \longrightarrow \operatorname{Pic}_{Y / k}^{\tau} \longrightarrow 0
$$

splits so the projection $\mathscr{P} i c^{\tau}(Y / k) \rightarrow \operatorname{Pic}_{Y / k}^{\tau}$ has a section and this yields the assertion.

\section{Remark 10.2.}

(1) Borne and Vistoli give an elegant independent proof of Proposition [10.1, using the dual Severi-Brauer variety (see [10, 13.2]).

(2) Proposition 10.1 provides a way to generate examples of smooth projective geometrically connected curves that satisfy Grothendieck's section conjecture, over any field with nontrivial Brauer group (see [10, section 13] for more details).

Remark 10.3. For any inflexible algebraic stack $X$ over a field $k$, one can define the abelianization $\Pi_{X / k}^{\mathrm{ab}}$ through a rigidification process. It comes with a natural morphism $\Pi_{X / k} \rightarrow \Pi_{X / k}^{\mathrm{ab}}$ and the composition $X \rightarrow \Pi_{X / k}^{\mathrm{ab}}$ is universal for morphisms from $X$ to a gerbe banded by a finite abelian group (see [9, App. A] or [11] for more details about the abelianized fundamental gerbe). The role played by the Albanese torsor $A_{\tau}^{1}(Y)$ in the proof of 10.1 is not surprising. Indeed, it turns out that $A_{\tau}^{1}(Y)$ is isomorphic to $\Pi_{Y / k}^{a b}$ : it is a finite gerbe (hence profinite), and it follows from 8.1 that the Albanese morphism $Y \rightarrow A_{\tau}^{1}(Y)$ is universal for maps from $Y$ to a gerbe banded by a finite abelian group. Hence it satisfies the same universal property as $Y \rightarrow \Pi_{Y / k}^{\mathrm{ab}}$ and in particular $A_{\tau}^{1}(Y) \simeq \Pi_{Y / k}^{\mathrm{ab}}$.

\section{Some VAnishing RESUlts FOR Ext SHEAVES}

In this section, we recall some vanishing or representability theorems for sheaves of the form $\mathscr{E} x t^{i}\left(G, \mathbb{G}_{\mathrm{m}}\right)$. In the following results, $S$ is a base scheme, and $G$ is a commutative group scheme over $S$. The sheaves $\mathscr{E} x t^{i}$ are computed as derived functors in the abelian category of fppf sheaves of commutative groups. Note that if $G$ and $H$ are such sheaves, then $\mathscr{E} x t^{i}(G, H)$ is also the fppf sheaf associated with the presheaf $T \mapsto \operatorname{Ext}_{T}^{i}\left(G \times{ }_{S} T, H \times \times_{S} T\right)$. With this description, it is clear that forming $\mathscr{E} x t^{i}(G, H)$ commutes with any base change $T \rightarrow S$. As mentioned on page 4 . $\mathscr{E} x t^{i}\left(G, \mathbb{G}_{\mathrm{m}}\right)$ will be denoted by $E^{i}(G)$.

Theorem 11.1 ([4, VIII prop. 3.3.1]). Assume that $G$ is finite and locally free over $S$, or that it is of finite type and of multiplicative type. Then $E^{1}(G)=0$.

Theorem 11.2 ([14, main theorem and Remark 3). Assume that $G$ is finite and flat over $S$, and that 2 is invertible in $S$. Then $E^{2}(G)=E^{3}(G)=0$.

Remark 11.3. If $S$ is the spectrum of a separably closed field of characteristic 2 , then it is known that $E^{2}\left(\alpha_{2}\right) \neq 0$ (see [16]).

Theorem 11.4. If $G$ is an abelian scheme over $S$, then $E^{1}(G)$ is representable by the dual abelian scheme $G^{t}$ [44, 17.6]. On the other hand, the Cartier dual $G^{D}$ is zero (obvious, e.g. use 3.21).

Corollary 11.5. Let $A$ be an abelian scheme and $G$ a duabelian group scheme over $S$. Then:

(i) For any $n \in \mathbb{N}^{*}$, the multiplication by $n$ in $E^{2}(A)$ is a monomorphism. If $2 \in \mathscr{O}_{S}^{\times}$, the multiplication by $n$ in $E^{i}(A)$ is an isomorphism for $i=2,3$ and a monomorphism for $i=4$.

(ii) If $F$ is a finite and locally free commutative group scheme, then any morphism from $F$ to $E^{2}(A)$ is trivial. If $2 \in \mathscr{O}_{S}^{\times}$, this is also true for $E^{3}(A), E^{4}(A)$ and $E^{2}(G)$.

(iii) If $S$ is regular and $2 \in \mathscr{O}_{S}^{\times}$, then $E^{2}(A)=E^{3}(A)=0$. 
Proof. The exact sequence of fppf sheaves

$$
0 \longrightarrow{ }_{n} A \longrightarrow A \stackrel{n}{\longrightarrow} A \longrightarrow 0
$$

induces an exact sequence:

$$
E^{i-1}\left({ }_{n} A\right) \longrightarrow E^{i}(A) \stackrel{n}{\longrightarrow} E^{i}(A) \longrightarrow E^{i}\left({ }_{n} A\right)
$$

But the scheme ${ }_{n} A$ is finite and locally free over $S$, hence $E^{1}\left({ }_{n} A\right)=0$ by 11.1 If $2 \in \mathscr{O}_{S}^{\times}$, by 11.2 the groups $E^{2}\left({ }_{n} A\right)$ and $E^{3}\left({ }_{n} A\right)$ also vanish. This gives (i). If $S$ is regular then $E^{2}(A)$ and $E^{3}(A)$ are torsion by [13, $\S 7$, whence (iii). In (ii), the question is local on $S$ so we may assume that $F$ is free of order $n$. But then it is killed by $n(50, \S 1])$ and the result follows from (i) for the morphisms to $E^{2}(A), E^{3}(A)$ or $E^{4}(A)$. Since $G$ is duabelian, by 2.16 and 11.2 the sheaf $E^{2}(G)$ is isomorphic to $E^{2}\left(A^{\prime}\right)$ where $A^{\prime}$ is an abelian scheme. Hence any morphism from $F$ to $E^{2}(G)$ is trivial by the previous case.

Theorem 11.6. Let $G$ be a finitely generated twisted constant group over $S$ (see 3.7). Then $E^{i}(G)=0$ for all $i>0$.

Proof. The question is local on $S$ so we may assume that $G$ is constant, associated to an ordinary abelian group $M$ of finite type. It suffices to consider the cases $M=\mathbb{Z}$ and $M=\mathbb{Z} / n \mathbb{Z}$. If $M=\mathbb{Z}$, the functor $\mathscr{H}$ om $(G,$.$) is the identity hence E^{i}(G)=0$ for all $i>0$. If $M=\mathbb{Z} / n \mathbb{Z}$, the result follows by considering the long exact sequence associated with the short exact sequence $0 \rightarrow \mathbb{Z} \rightarrow \mathbb{Z} \rightarrow \mathbb{Z} / n \mathbb{Z} \rightarrow 0$ (for $i=1$ the statement also follows from 11.1).

\section{REFERENCES}

[1] Schémas en groupes (SGA 3, new edition). Tomes I, III. Documents Mathématiques 7, 8. Séminaire de Géométrie Algébrique du Bois Marie 1962-64. A seminar directed by M. Demazure and A. Grothendieck with the collaboration of M. Artin, J.-E. Bertin, P. Gabriel, M. Raynaud and J-P. Serre. Revised and annotated editions of the 1970 French original.

[2] Schémas en groupes (SGA 3). Tome II: Groupes de type multiplicatif, et structure des schémas en groupes généraux. Séminaire de Géométrie Algébrique du Bois Marie 1962/64 (SGA 3). Dirigé par M. Demazure et A. Grothendieck. Lecture Notes in Mathematics, Vol. 152. Springer-Verlag, Berlin, 1962/1964.

[3] Théorie des topos et cohomologie étale des schémas. Tomes 1, 2, 3. Springer-Verlag, Berlin, 1972-1973. Séminaire de Géométrie Algébrique du Bois-Marie 1963-1964 (SGA 4), Dirigé par M. Artin, A. Grothendieck, et J. L. Verdier. Avec la collaboration de N. Bourbaki, P. Deligne et B. Saint-Donat, Lecture Notes in Mathematics, Vol. 269, 270, 305.

[4] Groupes de monodromie en géométrie algébrique. Springer-Verlag, Berlin, 1972-73. Séminaire de Géométrie Algébrique du Bois Marie - 1967-69 - (SGA 7), Lecture Notes in Mathematics, Vol. 288, 340.

[5] Revêtements étales et groupe fondamental (SGA 1). Documents Mathématiques (Paris) , 3. Société Mathématique de France, Paris, 2003. Séminaire de géométrie algébrique du Bois Marie 1960-61. Dirigé par A. Grothendieck, augmenté de deux exposés de Mme M. Raynaud. Édition recomposée et annotée du volume 224 des Lectures Notes in Mathematics publiée en 1971 par Springer-Verlag.

[6] Dan Abramovich and Angelo Vistoli. Compactifying the space of stable maps. J. Amer. Math. Soc., 15(1):27-75 (electronic), 2002.

[7] The Stacks Project Authors. Stacks Project.

[8] Luca Barbieri-Viale and Vasudevan Srinivas. Albanese and Picard 1-motives. Mém. Soc. Math. Fr. (N.S.), (87):vi+104, 2001.

[9] Indranil Biswas and Niels Borne. Tamely ramified torsors and parabolic bundles, 2017.

[10] Niels Borne and Angelo Vistoli. The Nori fundamental gerbe of a fibered category. J. Algebraic Geom., 24(2):311-353, 2015.

[11] Niels Borne and Angelo Vistoli. Fundamental gerbes. Algebra Number Theory, 13(3):531-576, 2019.

[12] Alexander Braverman and Roman Bezrukavnikov. Geometric Langlands correspondence for $\mathscr{D}$-modules in prime characteristic: the GL $(n)$ case. Pure Appl. Math. Q., 3(1, Special Issue: In honor of Robert D. MacPherson. Part 3):153-179, 2007.

[13] Lawrence Breen. Extensions of abelian sheaves and Eilenberg-MacLane algebras. Invent. Math., 9:15-44, $1969 / 1970$.

[14] Lawrence Breen. Un théorème d'annulation pour certains Ext ${ }^{i}$ de faisceaux abéliens. Ann. Sci. École Norm. Sup. (4), 8(3):339-352, 1975.

[15] Lawrence Breen. Bitorseurs et cohomologie non abélienne. In The Grothendieck Festschrift, Vol. I, volume 86 of Progr. Math., pages 401-476. Birkhäuser Boston, Boston, MA, 1990.

[16] Lawrence S. Breen. On a nontrivial higher extension of representable abelian sheaves. Bull. Amer. Math. Soc., $75: 1249-1253,1969$. 
[17] Sylvain Brochard. Foncteur de Picard d'un champ algébrique. Math. Ann., 343:541-602, 2009.

[18] Sylvain Brochard. Finiteness theorems for the Picard objects of an algebraic stack. Adv. Math., 229:1555-1585, 2012 .

[19] Charles Cadman. Using stacks to impose tangency conditions on curves. Amer. J. Math., 129(2):405-427, 2007.

[20] Tsao-Hsien Chen and Xinwen Zhu. Geometric Langlands in prime characteristic. Comp. Math., (153):395-452, 2017.

[21] Jean-Louis Colliot-Thélène and Jean-Jacques Sansuc. Torseurs sous des groupes de type multiplicatif; applications à l'étude des points rationnels de certaines variétés algébriques. C. R. Acad. Sci. Paris Sér. A-B, 282(18):Aii, A1113-A1116, 1976.

[22] Jean-Louis Colliot-Thélène and Jean-Jacques Sansuc. Variétés de première descente attachées aux variétés rationnelles. C. R. Acad. Sci. Paris Sér. A-B, 284(16):A967-A970, 1977.

[23] Jean-Louis Colliot-Thélène and Jean-Jacques Sansuc. La descente sur une variété rationnelle définie sur un corps de nombres. C. R. Acad. Sci. Paris Sér. A-B, 284(19):A1215-A1218, 1977.

[24] Jean-Louis Colliot-Thélène and Jean-Jacques Sansuc. La descente sur les variétés rationnelles. II. Duke Math. J., 54(2):375-492, 1987.

[25] Pierre Deligne. Théorie de Hodge. III. Inst. Hautes Études Sci. Publ. Math., (44):5-77, 1974.

[26] Ron Donagi and Tony Pantev. Torus fibrations, gerbes, and duality. Mem. Amer. Math. Soc., 193(901):vi+90, 2008. With an appendix by D. Arinkin.

[27] Ron Donagi and Tony Pantev. Langlands duality for Hitchin systems. Invent. Math., 189(3):653-735, 2012.

[28] Hélène Esnault, V. Srinivas, and Eckart Viehweg. The universal regular quotient of the Chow group of points on projective varieties. Invent. Math., 135(3):595-664, 1999.

[29] Gerd Faltings and Ching-Li Chai. Degeneration of abelian varieties, volume 22 of Ergebnisse der Mathematik und ihrer Grenzgebiete (3) [Results in Mathematics and Related Areas (3)]. Springer-Verlag, Berlin, 1990. With an appendix by David Mumford.

[30] Barbara Fantechi, Lothar Göttsche, Luc Illusie, Steven L. Kleiman, Nitin Nitsure, and Angelo Vistoli. Fundamental algebraic geometry, volume 123 of Mathematical Surveys and Monographs. American Mathematical Society, Providence, RI, 2005. Grothendieck's FGA explained.

[31] Jean Giraud. Cohomologie non abélienne. Springer-Verlag, Berlin, 1971. Die Grundlehren der mathematischen Wissenschaften, Band 179.

[32] Alexander Grothendieck. Éléments de géométrie algébrique. Inst. Hautes Études Sci. Publ. Math., (4, 8, 11, 17, 20, 24, 28, 32), 1960-1967.

[33] Alexander Grothendieck. Fondements de la géométrie algébrique. [Extraits du Séminaire Bourbaki, 19571962.]. Secrétariat mathématique, Paris, 1962.

[34] Alexander Grothendieck. Éléments de géométrie algébrique. I. Le langage des schémas, volume 166 of Grundlehren Math. Wiss. Springer-Verlag, 1971.

[35] Jack Hall. Cohomology and base change for algebraic stacks. Math. Z., 278(1-2):401-429, 2014.

[36] Luc Illusie. Complexe cotangent et déformations. II. Lecture Notes in Mathematics, Vol. 283. Springer-Verlag, Berlin, 1972.

[37] Peter Jossen. On the arithmetic of 1-motives. PhD Thesis. Webpage of the author, 2009.

[38] Gérard Laumon. Transformation de Fourier généralisée. arXiv:9603004, 1996.

[39] Gérard Laumon and Laurent Moret-Bailly. Champs algébriques, volume 39 of Ergebnisse der Mathematik und ihrer Grenzgebiete. 3. Folge. A Series of Modern Surveys in Mathematics [Results in Mathematics and Related Areas. 3rd Series. A Series of Modern Surveys in Mathematics]. Springer-Verlag, Berlin, 2000.

[40] Saunders Mac Lane. Categories for the working mathematician, volume 5 of Graduate Texts in Mathematics. Springer-Verlag, New York, second edition, 1998.

[41] Laurent Moret-Bailly. Pinceaux de variétés abéliennes. Astérisque, (129):266, 1985.

[42] D. Mumford, J. Fogarty, and F. Kirwan. Geometric invariant theory, volume 34 of Ergebnisse der Mathematik und ihrer Grenzgebiete (2) [Results in Mathematics and Related Areas (2)]. Springer-Verlag, Berlin, third edition, 1994.

[43] Martin Olsson. Algebraic spaces and stacks, volume 62 of American Mathematical Society Colloquium Publications. American Mathematical Society, Providence, RI, 2016.

[44] F. Oort. Commutative group schemes, volume 15 of Lecture Notes in Mathematics. Springer-Verlag, Berlin-New York, 1966.

[45] Niranjan Ramachandran. Duality of Albanese and Picard 1-motives. K-Theory, 22(3):271-301, 2001.

[46] Michel Raynaud. Faisceaux amples sur les schémas en groupes et les espaces homogènes. Lecture Notes in Mathematics, Vol. 119. Springer-Verlag, Berlin, 1970.

[47] Henrik Russell. Generalized Albanese and its dual. J. Math. Kyoto Univ., 48(4):907-949, 2008.

[48] Henrik Russell. Albanese varieties with modulus over a perfect field. Algebra Number Theory, 7(4):853-892, 2013.

[49] Stephen S. Shatz. Group schemes, formal groups, and p-divisible groups. In Arithmetic geometry (Storrs, Conn., 1984), pages 29-78. Springer, New York, 1986.

[50] John Tate and Frans Oort. Group schemes of prime order. Ann. Sci. École Norm. Sup. (4), 3:1-21, 1970.

IMAG, Université de Montpellier, CNRS, Montpellier, France

E-mail address: sylvain.brochard@umontpellier.fr 